\title{
Grupoides de Lie e o teorema de Noether na formulação lagrangiana da teoria clássica de campos
}

\author{
Luiz Henrique Pereira Pêgas
}

\author{
TESE APRESENTADA \\ AO
Instituto de Matemática E Estatística \\ DA \\ Universidade De SÃo PaUlo \\ PARA \\ OBTENÇÃO DO TÍTULO \\ DE \\ Doutor EM CIÊNCIAS \\ Programa: Matemática Aplicada \\ Orientador: Prof. Dr. Frank Michael Forger
}

Durante o desenvolvimento deste trabalho o autor recebeu auxílio financeiro da CAPES

São Paulo, julho de 2014 



\section{Grupoides de Lie e o teorema de Noether na formulação lagrangiana da teoria clássica de campos}

Esta versão da tese contém as correções e alterações sugeridas pela Comissão Julgadora durante a defesa da versão original do trabalho, realizada em 12/09/2014. Uma cópia da versão original está disponível no Instituto de Matemática e Estatística da Universidade de São Paulo.

Comissão Julgadora:

- Prof. Dr. Frank Michael Forger (orientador) - IME-USP

- Prof. Dr. Ivan Struchiner - IME-USP

- Prof. Dr. Hugo Luiz Mariano - IME-USP

- Prof. Dr. Henrique Bursztyn - IMPA

- Prof. Dr. Eduardo Outeiral Correa Hoefel - UFPR 
À Aline, a quem dedico tudo o que faço e a quem devo o que hoje sou. 
"The very young do not always do as they are told."

Anteaus

"Stargate SG-1"

"As verdadeiras relações entre esses objetos reais são a única realidade que podemos atingir, e a única condição para isso é que as relações entre esses objetos sejam as mesmas que existem entre as imagens que somos obrigados a pôr em seu lugar. Se conhecemos essas relações, pouco importa que julguemos ser conveniente substituir uma imagem por uma outra." H. Poincaré (sobre as teorias da Física Moderna) "A Ciência e a Hipótese"

"It's better to burn out than to fade away" Neil Young "Hey Hey, My My (Into the Black)" 


\section{Agradecimentos}

Agradeço ao meu orientador, prof. Dr. Michael Forger, não apenas a oportunidade de trabalhar a seu lado, mas a liberdade que me foi dada para perseguir meus pontos de vista. Ao programa de Doutorado em Matemática Aplicada do IME-USP, que tornou este trabalho possível. À CAPES, o financiamento recebido. Ao prof. Dr. Eduardo Hoefel, o suporte e acompanhamento ao longo do curso. Ao grupo de pesquisa em física-matemática, as discussões e o espaço aberto a apresentações. Em especial, a Bruno T. Costa, que apontou erros nas versões preliminares deste trabalho; as discussões que tivemos ajudaram a aumentar minha própria compreensão sobre o tema. Ao prof. Dr. Hugo Luiz Mariano, que me abriu as portas para o maravilhoso mundo das categorias, ajudando-me a compreender algumas questões sobre fundamentos da matemática que há anos me incomodavam (várias ainda me incomodam - minha culpa). Ao prof. Dr. Odilon Luciano, as conversas de corredor, sempre iluminantes. Ao prof. Dr. Ivan Struchiner, que me apontou referências cruciais ao desenvolvimento deste trabalho. Aos demais membros da comissão julgadora, as sugestões que, sem sombra de dúvida, ajudaram a melhorar a qualidade desta tese. E à minha família, o suporte e compreensão. 


\section{Resumo}

Pêgas, L.H.P. Grupoides de Lie e o teorema de Noether na formulação lagrangiana da teoria clássica de campos. 2014. 74 p. Tese (Doutorado) - Instituto de Matemática e Estatística, Universidade de São Paulo, São Paulo, 2014.

O objetivo desta tese é oferecer um arcabouço que permita a modelagem de simetrias em fibrados suaves, que possuam um bom comportamento local. Para tanto, usa-se ferramentas de grupoides de Lie e correlatas, com a finalidade de reduzir, quando possível, simetrias dadas pela ação de um grupo diferenciável, possivelmente de dimensão infinita, sobre um fibrado suave, a problemas em dimensão finita. Uma definição de invariância de uma forma diferencial, definida no espaço total de um fibrado suave, sob a ação de um grupoide de Lie, é apresentada e desenvolvida. A seguir, discute-se estas ferramentas no contexto da formulação lagrangiana da teoria clássica de campos com o objetivo de descrever, simultaneamente, simetrias internas e no espaço-tempo, de maneira unificada. Obtém-se então, nesta linguagem, alguns objetos de estudo centrais da teoria, como os teoremas de Noether e, no caso das teorias de calibre, os teoremas de acoplamento mínimo e Utiyama. Por fim, discute-se brevemente o caso de simetrias a menos de elementos de contato e divergências totais.

Palavras-chave: grupoides, simetrias, teoria clássica de campos 


\section{Abstract}

Pêgas, L.H.P. Lie groupoids and Noether's theorem in the Lagrangian formalism of classical field theory. 2014. 74 p. Thesis (Ph.D.) - Instituto de Matemática e Estatística, Universidade de São Paulo, São Paulo, 2014.

The aim of this thesis is to provide a framework that allows the modelling of symmetries in smooth fibre bundles which have good local behaviour. For that, we use Lie groupoids and related tools in order to reduce, whenever possible, symmetries given by the action of a possibly infinite dimensional differentiable group on a smooth fibre bundle to finite dimensional problems. We give a definition of invariance of a differential form, defined on the total space of a fibre bundle, by the action of a Lie groupoid. Then, we discuss these tools in the case of a Lagrangian classical field theory to describe internal and space-time symmetries simultaneously, in a unified way. With this language, we get some central objects of the theory such as Noether's theorems and, in the case of gauge theories, the minimal coupling and Utiyama's theorems. Lastly, we briefly discuss the case of symmetries up to contact elements and a total divergence.

Keywords: groupoids, symmetries, classical field theory 


\section{Sumário}

$\begin{array}{ll}\text { Proêmio } & 1\end{array}$

1 Fundamentos categoriais $\quad 3$

1.1 Conceitos preliminares . . . . . . . . . . . . . . . . . 3

1.2 Estruturas internas a uma categoria . . . . . . . . . . . . . 7

2 Categorias e grupoides internos à categoria de variedades suaves $\quad 21$

2.1 Grupoides de Lie e estruturas derivadas . . . . . . . . . . . . . . . . . . . . . 21

2.2 Algebroides de Lie . . . . . . . . . . . . . . . . . . . . . . 31

2.3 Um pouco sobre fibrados principais e associados . . . . . . . . . . . . . . 34

3 Simetrias em teoria clássica de campos, na formulação lagrangiana $\quad 45$

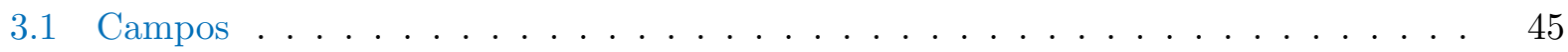

3.2 Teorias de calibre . . . . . . . . . . . . . . . . . . . 51

3.3 Simetrias em um sentido mais amplo . . . . . . . . . . . . . 54

$\begin{array}{ll}\text { Epílogo } & 57\end{array}$

$\begin{array}{ll}\text { Referências Bibliográficas } & \mathbf{5 9}\end{array}$ 


\section{Proêmio}

Este é um trabalho sobre fundamentos. Nas últimas décadas, o foco sobre a fundamentação da matemática por meio de uma teoria de conjuntos lentamente derivou-se para o da teoria das categorias. Enquanto a primeira enfatiza os indivíduos em si, a segunda destaca a relação entre os indivíduos, criando uma espécie de sociologia para entidades matemáticas. Esta mudança de paradigma favorece a organização dos tópicos estudados em estruturas, que passam a ser objetos de estudo por si só. Deste modo, destacam-se padrões, gabaritos de teorias que fluorescem relações entre partes da ciência que antes pareciam desconexas. Das ciências naturais, a que mais faz uso das técnicas desenvolvidas na matemática é, sem dúvida, a física. Porém, por este mesmo motivo, as mudanças de paradigmas na matemática levam tempo para serem "absorvidas" pela física. Aqui, faremos presente uma pequena contribuição nesta direção, cujas consequências aparentam fortes o suficiente para justificar o esforço de uma mudança estrutural - o uso de técnicas categoriais para abordar alguns pontos da teoria clássica de campos, em nosso caso, simetrias em sistemas lagrangianos.

É importante notar que estes são primeiros passos. Muito há a ser feito ainda na direção de aplicações verdadeiras da teoria das categorias nas ciências em geral e, ao menos para a física, muitos resgates de ideias de autores passados são necessários (se não, ao menos muito convenientes). Citarei dois destes conjuntos de ideias. O primeiro deles é a obra de Charles Ehresmann. Ao invés de citar inúmeros trabalhos de Ehresmann, o que é feito nas referências que cito, achei conveniente mencioná-lo explicitamente nesta introdução, não por um ou outro artigo, mas pela maneira como pensava. Ao delinear a geometria diferencial moderna, aos poucos foi-se perdendo o sabor categorial impresso por Ehresmann e o tratamento de estruturas foi o mais afetado com isto. Como exemplo, a teoria clássica dos campos se fia, hoje, na geometria diferencial. No tratamento geral de simetrias, o uso de grupoides me parece inevitável. As noções de ações de grupoides, prolongamentos de grupoides e de suas ações, pseudogrupos de transformações, etc., foram todas detalhadas em seus trabalhos e mostrar que isso simplifica o tratamento formal de simetrias em teoria clássica de campos é um dos objetivos desta tese. Seria injusto mencionar um ou dois de seus trabalhos e impossível mencionar todos, um a um. O outro conjunto de ideias está relacionado à maneira como encaramos a construção da geometria diferencial. Trabalhar diretamente com quantidades infinitesimais, como faziam S. Lie e E. Cartan, é tão quotidiano para um físico que a maioria das vezes esquece-se o fato de que isto é virtualmente proibido na formulação padrão da geometria diferencial moderna. Isto causa uma distorção na formulação de teorias como a teoria clássica dos campos, na qual se pensa uma coisa, mas se escreve outra. Acredito que lidar diretamente com infinitesimais simplifica a formulação da teoria a ponto de iluminar questões que, sem isto, seriam permanentemente obscuras. Hoje, este resgate é liderado por pesquisadores como A. Kock, com seus trabalhos sobre geometria diferencial sintética [22; 23], com contribuições extremamente relevantes, em minha opinião, para a reformulação adequada de teorias físicas, tendo em vista trabalhos que trazem modelos bem adaptados, como o texto [32], de I. Moerdijk e G. Reyes. Entretanto, não faremos isso aqui. Apesar de reconhecer esta quase necessidade, escrever este trabalho em termos da geometria diferencial sintética seria uma guinada tão radical que arriscaria torná-lo ilegível para a quase totalidade do público que mais se beneficiaria com isso: os físicos.

Pensando no equilíbrio entre a necessidade de mudar a linguagem e a legibilidade do trabalho, este texto foi dividido em três partes. No capítulo 1 são delineados alguns conceitos chave da teoria de categorias, para que o leitor leigo tenha pontos de referência para se familiarizar com a 
linguagem e para que o leitor experiente saiba qual abordagem adotamos, das muitas possíveis, a cada um deles. Não é um capítulo de leitura essencial para aqueles com conhecimento na área de grupoides e algebroides de Lie mas, como também tem o propósito de fixar notações, sua leitura é recomendável. Além disso, um ponto estrutural é ali defendido. Apesar de ser diagramaticamente equivalente definir categorias internas por meio de objetos e morfismos e por meio de morfismos apenas, é a segunda formulação que mostra a independência do contexto, ou seja, que permite formular categorias internas sem referência a propriedades que seus morfismos estruturais devam satisfazer, dependendo do ambiente onde se encontram. Como exemplo, cito grupoides de Lie que, em sua formulação tradicional, exige-se que seus morfismos domínio e codomínio (ou fonte e alvo), sejam submersões sobrejetoras. Obviamente, o conceito de submersão sobrejetora não é transferível para outras categorias quaisquer - ele depende do contexto (a categoria das aplicações suaves entre variedades suaves). Na presente formulação, fica claro que isto é uma consequência e não um requisito, bem como a redundância da exigência de uma seção de unidades. Grupoides podem então ser vistos como um objeto e estruturas que contém em si um sub-objeto invariante por tais estruturas; é uma espécie de extensão de sua base. Visando futuras aplicações destas técnicas, neste capítulo nenhum resultado faz qualquer referência a categorias específicas. Mantivemos seu desenvolvimento em um nível o mais geral possível, independente de contexto, para fixar a ideia de que a passagem de uma teoria algébrica, como a teoria de monoides ou a teoria de grupos, para uma teoria essencialmente algébrica, como a teoria de categorias ou a teoria de grupoides, é não apenas bem comportada, como informativa. Inclusive, acredito ser este o mesmo tipo de passagem que se faz da mecânica clássica para a teoria dos campos. Uma vez entendido o conceito de grupoides, a analogia é cristalina.

No capítulo 2 é apresentada, resumidamente, a teoria de grupoides de Lie, necessária para nossos propósitos. Ali, são explanados conceitos dependentes do contexto das variedades suaves, como prolongamentos a jatos de ações e de grupoides de Lie, algebroides de Lie e uma releitura de alguns tópicos da teoria de fibrados principais e seus associados em termos da linguagem de grupoides de Lie, que acreditamos não terem sido suficientemente bem explorados na literatura padrão. A contribuição deste capítulo que, até onde pude investigar, não está presente na literatura até o momento, é o conceito de forma invariante com relação a uma ação de um grupoide de Lie. Tal conceito é central, pois permite que descrevamos em dimensão finita, a ação de alguns grupos de dimensão infinita sobre fibrados, realizados como grupos de bisseções de grupoides de Lie. Porém, para aplicações em teoria clássica de campos, este conceito é insuficiente e deve ser adaptado, como feito no próximo capítulo.

No capítulo 3, pontos principais da teoria clássica de campos, em sua formulação lagrangiana, são abordados. Primeiro, é apresentado um resumo de uma formulação matemática adequada a uma teoria clássica de campos, com um conceito relativamente ingênuo de simetria e suas consequências. Depois, tomamos o caso especial das teorias de calibre, nas quais a linguagem de grupoides de Lie expressa todo o seu poder. Finalmente, é discutido o conceito de simetria em um sentido mais amplo, mais adequado ao caráter funcional que as quantidades invariantes devem exibir. A contribuição deste capítulo é a descrição formal de simetrias internas e no espaço-tempo, simultaneamente, por objetos que, localmente, têm dimensão finita.

A elaboração conceitual deste trabalho ocorreu mais ou menos de modo reverso ao descrito aqui. Em [13] os autores descrevem simetrias internas em uma teoria de calibre em termos de ações de fibrados de grupos. Lá, observam que, para descrever conjuntamente simetrias internas e no espaço-tempo, a necessidade de uma passagem de fibrados de grupos a grupoides de Lie era evidente. E foi com esta afirmação em mente que iniciei meus estudos em teoria de categorias, sem imaginar que sua relação com a física, e posteriormente com outras ciências naturais, fosse tão mais profunda, com um potencial esclarecedor tão grande. 


\section{Capítulo 1}

\section{Fundamentos categoriais}

\subsection{Conceitos preliminares}

Dado o caráter fundamental da teoria das categorias, é praticamente impossível, ou mesmo indesejável, obtermos uniformidade de notações dentre as várias áreas que dela fazem uso. Assim, é necessário que discorramos sobre algumas estruturas das quais necessitaremos, para que fixemos notações e pontos de vista adotados. Portanto, a apresentação feita aqui é absolutamente limitada e não tem a pretensão de resumir o conteúdo desta teoria. Sempre que necessário, indicaremos referências bibliográficas, nas quais o leitor poderá encontrar conteúdo didático, caso necessite.

A abordagem adotada aqui é próxima à de considerar os diagramas e soluções de problemas universais como axiomas, que podem ou não serem satisfeitos, dependendo do contexto, ou ambiente, sobre o qual são modelados. ${ }^{1}$ Deste modo, desenvolveremos a teoria de maneira diagramática tanto quanto possível, para só então aplicá-la a nosso ambiente de interesse, a saber, a categoria das aplicações suaves entre variedades diferenciáveis. Neste espírito, definiremos primeiramente o conceito de categoria de maneira direta (próxima a uma linguagem de primeira ordem), para podermos definir os diagramas limite necessários à construção do conceito de categoria interna a um ambiente (objeto de categoria em uma categoria). O conceito de categoria a seguir é uma variante do apresentado em [14].

Definição 1.1.1. Uma categoria consiste de indivíduos $f, g, h, \ldots$, ditos morfismos, duas letras funcionais $\Sigma$ e $T$, e uma letra funcional parcial $\mu$, satisfazendo:

1. $\forall f(T \Sigma f=\Sigma f)$;

2. $\forall f(\Sigma T f=T f)$;

3. $\forall g \forall f(\exists h(\mu(g, f)=h) \Leftrightarrow(\Sigma g=T f))$;

4. $\forall f(\mu(f, \Sigma f)=f)$;

5. $\forall f(\mu(T f, f)=f)$;

6. $\forall g \forall f(\exists h(\mu(g, f)=h) \Rightarrow(\Sigma \mu(g, f)=\Sigma f))$;

7. $\forall g \forall f(\exists h(\mu(g, f)=h) \Rightarrow(T \mu(g, f)=T g))$;

8. $\forall h \forall g \forall f((\Sigma h=T g) \wedge(\Sigma g=T f) \Rightarrow(\mu(\mu(h, g), f)=\mu(h, \mu(g, f))))$.

Um homomorfismo ${ }^{2}$ para estas estruturas é dito funtor (covariante) entre duas categorias.

\footnotetext{
${ }^{1}$ Discussões sobre esta abordagem, referida usualmente como "esboço", podem ser encontradas, por exemplo, em [19] ou em [5].

${ }^{2}$ Aqui, homomorfismo tem o sentido usual de homomorfismo de estruturas sobre uma linguagem formal. O leitor poderá encontrar definições e detalhes em [18].
} 
A definição anterior, apesar de imprecisa, pode ser reescrita como uma linguagem de primeira ordem sem muito esforço (mas será consideravelmente mais longa), cuja interpretação em uma teoria de conjuntos razoável fornecerá o que comumente se conhece por categorias concretas. Tal nível de detalhes é desnecessário para nossos propósitos. Entretanto, convém fixar de agora em diante uma teoria de conjuntos. Salvo menção explícita, quando nos referirmos a questões conjuntistas, usaremos a teoria de conjuntos de Zermelo-Fraenkel, com o axioma da escolha, abreviado por ZFC.

Exemplo 1.1.1. O leitor não deve ter dificuldade em mostrar que são categorias ${ }^{3}$ :

1. Set: os indivíduos são funções entre conjuntos de ZFC, $\Sigma$ toma a função identidade no domínio, $T$ toma a função identidade no codomínio e $\mu$ é a composta de funções;

2. Cat: os indivíduos são funtores entre categorias concretas (pequenas), $\Sigma$ toma o funtor identidade no domínio, $T$ toma o funtor identidade no codomínio e $\mu$ é a composta de funtores;

3. Man: os indivíduos são aplicações infinitamente diferenciáveis entre variedades suaves (variedades Hausdorff, com base topológica enumerável e localmente euclideanas, de dimensão $n), \Sigma$ toma a aplicação identidade no domínio, $T$ toma a aplicação identidade no codomínio e $\mu$ é a composta de aplicações. $\diamond$

Observação 1.1.1. Note que categorias, geralmente, versam sobre totalidades. Portanto, ao falarmos de categorias concretas, devemos tomar o cuidado de usar teorias de conjunto diferentes, ou em níveis diferentes, para expressar continente e conteúdo, sob o risco de introduzirmos paradoxos que trivializariam a teoria, como nos exemplos de Set e Cat acima. Uma pequena discussão sobre isto pode ser encontrada em [30] e em [4]. Note, entretanto, que isto não ocorre em Man. Devido ao teorema do mergulho de Whitney, Man é uma categoria "essencialmente pequena". $\diamond$

Definição 1.1.2. Seja $\mathbf{C}$ uma categoria. Dizemos que um morfismo e $\in \mathbf{C}$ é uma identidade se, e somente se, satisfizer

$$
\forall f \forall g(\exists \mu(e, f) \Rightarrow \mu(e, f)=f) \wedge(\exists \mu(g, e) \Rightarrow \mu(g, e)=g)
$$

Observação 1.1.2. Tradicionalmente, categorias são apresentadas como estruturas sobre duas coleções distintas: morfismos e objetos. Na abordagem adotada aqui, a noção de objeto de uma categoria pode ser recuperada ao notarmos que, na abordagem tradicional, existe uma bijeção entre objetos e morfismos identidade. Assim, objetos são totalmente codificados pelas identidades, que aqui são obtidas pela propriedade $e=\Sigma e=T e$. É fácil ver que tal coleção não é vazia e que, para cada morfismo $f$, há sempre duas identidades a ele associadas, a domínio $\Sigma f$ e a codomínio $T f$. $\diamond$

Notação 1.1.1. No que segue, denotaremos morfismos de uma categoria por $f: A \rightarrow B$, ou por $A \stackrel{f}{\rightarrow} B$, onde $A=\Sigma f$ e $B=T f$. Também, quando não houver risco de confusão, denotaremos $\mu(g, f)$ por $g \circ f$, ou simplesmente por $g f . \diamond$

Definição 1.1.3. Se $\mathbf{C}$ for uma coleção de morfismos de uma categoria, com $\Sigma, T$ e $\mu$ como na definição, podemos considerar a categoria oposta a $\mathbf{C}$, denotada por $\mathbf{C}^{o p}$, como a categoria obtida de $\mathbf{C}$ pela inversão formal da direção dos morfismos de $\mathbf{C}$, ou seja, pela troca de dominios por codominios e de codominios por domínios, fixadas as identidades. Dadas duas categorias $\mathbf{C} e$ $\mathbf{D}$, um funtor de $\mathbf{C}^{o p}$ em $\mathbf{D}$ é dito funtor contravariante de $\mathbf{C}$ em $\mathbf{D}$.

Definição 1.1.4. Sejam $\mathbf{C} e \mathbf{D}$ duas categorias. Um funtor $F: \mathbf{C} \rightarrow \mathbf{D}$ é dito

- pleno se, dadas identidades $A, B \in \mathbf{C}$, todo morfismo $h: F(A) \rightarrow F(B)$ estiver na imagem de $F$;

\footnotetext{
${ }^{3}$ É interessante notar que a escola de Ehresmann nomeia as categorias por seus morfismos, e não por seus objetos. Assim, mais correto seria nos referirmos a Set como a categoria de funções - Func - e não a categoria de conjuntos. Optamos aqui por manter a compatibilidade com a literatura, em sua maioria.
} 
- fiel se, dadas identidades $A, B \in \mathbf{C}$ e morfismos $f, g: A \rightarrow B$, tais que $F(f)=F(g)$ então, necessariamente, $f=g$;

- plenamente fiel, se for pleno e fiel;

- essencialmente sobrejetor em objetos, se todo objeto de $\mathbf{D}$ for isomorfo ${ }^{4}$ a algum objeto na imagem de F.

Definição 1.1.5. Dizemos que uma categoria $\mathbf{C}$ é sobre (ou estruturada sobre) a categoria $\mathbf{D}$, se existir um funtor covariante e fiel $F: \mathbf{C} \rightarrow \mathbf{D}$. Caso $\mathbf{D}=$ Set, dizemos que $\mathbf{C}$ é uma categoria concreta.

Definição 1.1.6. Dizemos que uma categoria $\mathbf{C}$ é uma subcategoria de uma categoria $\mathbf{D}$, se existir um funtor $F: \mathbf{C} \rightarrow \mathbf{D}$ que seja fiel e injetor em identidades. Se, além disso, for pleno, dizemos que $\mathbf{C}$ é subcategoria plena de $\mathbf{D}$. Dizemos que $\mathbf{C}$ é subcategoria ampla de $\mathbf{D}$, se for subcategoria, com F bijetor em identidades.

Definição 1.1.7. Dados $F, G: \mathbf{C} \rightarrow \mathbf{D}$ dois funtores covariantes, uma transformação natural de $F$ em $G$ é uma função $\alpha: \mathbf{C} \rightarrow \mathbf{D}$, tal que

$$
\forall g \forall f\left(\exists h\left(\mu_{\mathbf{C}}(g, f)=h\right) \Rightarrow \mu_{\mathbf{D}}(\alpha(g), F(f))=\alpha\left(\mu_{\mathbf{C}}(g, f)\right)=\mu_{\mathbf{D}}(G(g), \alpha(f))\right)
$$

De agora em diante, usaremos pesadamente o conceito de diagrama comutativo em uma categoria, bem como os conceitos de diagramas limite e diagramas colimite. Admitimos que o leitor tenha certa familiaridade com tais conceitos, que podem ser encontrados em inúmeras fontes. Nossa referência principal é [30]. Nesta mesma referência, há uma ampla discussão sobre a obtenção de conceitos duais pelo processo de inversão da direção de morfismos, mencionada na construção da categoria oposta. Sugerimos que o leitor atente a isto, pois os conceitos de covariância e contravariância, de dual (ou oposta) a uma construção, serão, aqui, ubíquos.

Descrevamos alguns morfismos e limites notáveis que utilizaremos. Faremos isto apenas para o caso binário, mas o leitor deve ter em mente que são possíveis limites arbitrários (ver [30]). Fixemos uma categoria $\mathbf{C}$.

Definição 1.1.8. Dizemos que um morfismo $m$ é um monomorfismo quando, para todo par $f, g$ de morfismos tais que $m f=m g$, tivermos $f=g$. A situação dual é dita epimorfismo, ou seja, um morfismo e, tal que, para todo par $f, g$ de morfismos, com $f e=g e$, necessariamente tenhamos $f=g$. Um morfismo é dito isomorfismo, quando admitir inversa à esquerda e à direita. Neste caso, as inversas laterais são únicas e coincidem, permitindo que nos refiramos a este como o isomorfismo inverso.

Definição 1.1.9. Dados $A$ e $B$, objetos de $\mathbf{C}$, chamamos de produto de $A$ e $B$ a um diagrama em $\mathbf{C}$, da forma $A \stackrel{\langle A, B\rangle_{1}}{\longleftarrow} A \times B \stackrel{\langle A, B\rangle_{2}}{\longrightarrow} B$, tal que, para todo diagrama da forma $A \stackrel{\varphi}{\longleftarrow} C \stackrel{\psi}{\longrightarrow} B$, exista um único morfismo $(\varphi, \psi)_{\langle A, B\rangle}: C \rightarrow A \times B$, com a propriedade de fatoração

$$
\begin{aligned}
& \langle A, B\rangle_{1} \circ(\varphi, \psi)_{\langle A, B\rangle}=\varphi \\
& \langle A, B\rangle_{2} \circ(\varphi, \psi)_{\langle A, B\rangle}=\psi
\end{aligned}
$$

onde $\langle A, B\rangle_{i}$ é dita i-ésima projeção do produto. Por vezes, denotaremos o morfismo canônico simplesmente por $(\varphi, \psi)$.

$A$ estrutura dual a esta é dita coproduto, e denotada por $A \stackrel{\langle A, B\rangle^{1}}{\longrightarrow} A \sqcup B \stackrel{\langle A, B\rangle^{2}}{\longleftarrow} B$. Também, podemos denotar $A+B$, ao invés de $A \sqcup B$.

\footnotetext{
${ }^{4}$ Veja a definição 1.1 .8
} 
Observação 1.1.3. Diagramaticamente, descrevemos a situação acima assim:

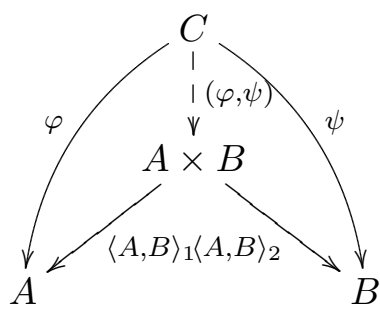

Definição 1.1.10. Dados morfismos $f$ e $g$, dizemos que o morfismo $h$ iguala $f$ e $g$, quando $f h=g h$. Dizemos que $\varepsilon$ é um equalizador de $f$ e $g$, quando $\varepsilon$ igualar $f$ e $g$ e ainda, para todo $h$ que iguale $f$ e g, existir um único morfismo $\bar{h}$, tal que $h=\varepsilon \circ \bar{h}$. A estrutura dual a esta é dita coequalizador.

Observação 1.1.4. Diagramaticamente, descrevemos a situação acima assim:

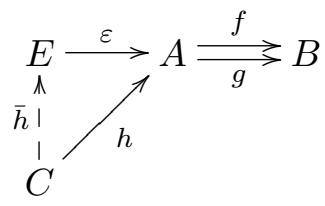

$\diamond$

Definição 1.1.11. Dado um diagrama do tipo $A \stackrel{f}{\rightarrow} C \stackrel{g}{\longleftarrow} B$, o pull-back de $f$ e g, é um diagrama $A \stackrel{\langle f, g\rangle_{1}}{\longleftarrow} A_{f} \times{ }_{g} B \stackrel{\langle f, g\rangle_{2}}{\longrightarrow} B$, tal que $f\langle f, g\rangle_{1}=g\langle f, g\rangle_{2}$ e, para todo diagrama da forma $A \stackrel{\varphi}{\longleftarrow} D \stackrel{\psi}{\longrightarrow} B$, com $f \varphi=g \psi$, exista um único morfismo $(\varphi, \psi)_{\langle f, g\rangle}: D \rightarrow A_{f} \times_{g} B$, satisfazendo a condição de fatoração

$$
\begin{aligned}
& \langle f, g\rangle_{1} \circ(\varphi, \psi)_{\langle f, g\rangle}=\varphi \\
& \langle f, g\rangle_{2} \circ(\varphi, \psi)_{\langle f, g\rangle}=\psi
\end{aligned}
$$

$A$ estrutura dual a esta é dita push-out, e denotada por $A \stackrel{\langle f, g\rangle^{1}}{\longleftrightarrow} A_{f} \sqcup_{g} B \stackrel{\langle f, g\rangle^{2}}{\longleftarrow} B$.

Observação 1.1.5. Diagramaticamente, descrevemos a situação acima assim:

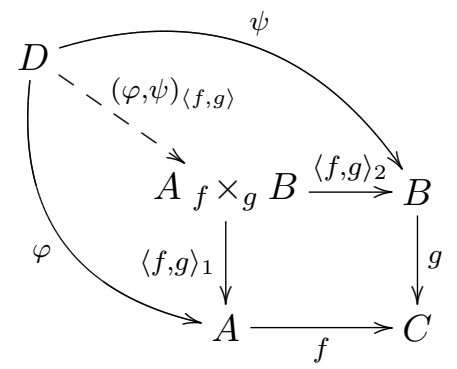

$\diamond$

Em um primeiro momento, a notação para as projeções e para os morfismos canônicos pode parecer exageradamente carregada. A notação aqui apresentada visa eliminar ambiguidades e estabelecer um padrão para que possamos, apenas pelo nome de um morfismo canônico, dizer quais são suas propriedades, quais são as condições necessárias para sua boa definição e seus aspectos computacionais. Isto será de grande ajuda, quando tivermos de lidar com compostas de várias projeções diferentes, oriundas de vários pull-backs diferentes. 
Definição 1.1.12. Dizemos que um objeto 1 é terminal em $\mathbf{C}$ quando, para todo objeto $A$ de $\mathbf{C}$ existir um único morfismo $!_{A}: A \rightarrow 1$. A estrutura dual a esta é dita objeto inicial, denotada geralmente por $!_{A}: 0 \rightarrow A$. Se um objeto for simultaneamente terminal e inicial, dizemos que é nulo.

Observação 1.1.6. Os limites (e colimites) notáveis, acima definidos, podem ou não existir, dependendo da categoria em questão. Entretanto, quando existirem, serão únicos a menos de isomorfismos canônicos destas estruturas. $\diamond$

Notação 1.1.2. Dados $A$ e $B$, objetos de uma categoria $\mathbf{C}$, denotaremos:

1. $\operatorname{Hom}_{\mathbf{C}}(A, B)$ como a coleção dos morfismos $f$ de $\mathbf{C} \operatorname{com} \Sigma f=A$ e $T f=B$;

2. End $_{\mathbf{C}}(A)$ como a coleção dos morfismos $f$ de $\mathbf{C}$ com $\Sigma f=T f=A$, ditos endomorfismos de $A$;

3. $\operatorname{Iso}_{\mathbf{C}}(A, B)$ como a coleção dos isomorfismos $f$ de $\mathbf{C} \operatorname{com} \Sigma f=A$ e $T f=B$;

4. $\operatorname{Aut}_{\mathbf{C}}(A)$ como a coleção dos isomorfismos $f$ de $\mathbf{C} \operatorname{com} \Sigma f=T f=A$, ditos automorfismos de $A . \diamond$

\subsection{Estruturas internas a uma categoria}

Nesta seção, apresentaremos as estruturas básicas com as quais lidaremos em termos de diagramas em uma dada categoria "ambiente". A ideia é que os diagramas sirvam como gabaritos, que podem ou não fazer sentido, dependendo do contexto, ou seja, da categoria ambiente. Isto é próximo à ideia de considerar diagramas como axiomas e sua "realização" em uma dada categoria como um modelo para estes axiomas, grosseiramente falando. Assim, toda vez que formos apresentar um conceito em termos de um dado limite, como um pull-back por exemplo, o leitor é convidado a adicionar um "caso exista" às condições apresentadas. Deste modo, poderemos prosseguir com a teoria, ao máximo possível, de maneira independente do contexto (do modelo, ou ainda, da categoria ambiente). ${ }^{5}$

Definição 1.2.1. Sejam $\mathbf{C}$ uma categoria, $C$ um objeto de $\mathbf{C}, \Sigma, T \in \operatorname{End}_{\mathbf{C}}(C) e$ $\mu \in \operatorname{Hom}_{\mathbf{C}}\left(C{ }_{\Sigma} \times_{T} C, C\right)$. Denote a identidade relativa ao objeto $C$ por id $C$. Dizemos que $(C, \Sigma, T, \mu)$ é uma categoria interna a $\mathbf{C}$ (ou categoria em $\mathbf{C}$, ou ainda, objeto de categoria em $\mathbf{C}$ ), se estes dados satisfizerem:

i) $T \Sigma=\Sigma e \Sigma T=T$;

ii) $T \mu=T\langle\Sigma, T\rangle_{1}$

iii) $\Sigma \mu=\Sigma\langle\Sigma, T\rangle_{2}$;

iv) $\mu\left(i d_{C}, \Sigma\right)_{\langle\Sigma, T\rangle}=i d_{C}$ e $\mu\left(T, i d_{C}\right)_{\langle\Sigma, T\rangle}=i d_{C}$;

v) existem os pull-backs $\left(C_{\Sigma} \times_{T} C\right)_{\Sigma\langle\Sigma, T\rangle_{2}} \times_{T} C$ e $C_{\Sigma} \times_{T\langle\Sigma, T\rangle_{1}}\left(C_{\Sigma} \times_{T} C\right)$;

vi)

$$
\begin{aligned}
\mu\left(\mu\left\langle\Sigma\langle\Sigma, T\rangle_{2}, T\right\rangle_{1},\left\langle\Sigma\langle\Sigma, T\rangle_{2}, T\right\rangle_{2}\right)_{\left\langle\Sigma\langle\Sigma, T\rangle_{2}, T\right\rangle} & \approx \\
& \approx \mu\left(\left\langle\Sigma, T\langle\Sigma, T\rangle_{1}\right\rangle_{1}, \mu\left\langle\Sigma, T\langle\Sigma, T\rangle_{1}\right\rangle_{2}\right)_{\left\langle\Sigma, T\langle\Sigma, T\rangle_{1}\right\rangle}
\end{aligned}
$$

onde o símbolo $\approx$ é necessário devido ao fato de que, em geral, temos apenas um isomorfismo (canônico) entre $\left(C_{\Sigma} \times_{T} C\right)_{\Sigma\langle\Sigma, T\rangle_{2}} \times_{T} C$ e $C_{\Sigma} \times_{T\langle\Sigma, T\rangle_{1}}\left(C_{\Sigma} \times_{T} C\right)$, e usamos este símbolo para indicar que o diagrama comuta através deste isomorfismo;

\footnotetext{
${ }^{5} \mathrm{O}$ leitor poderá encontrar mais informações sobre categorias internas em, por exemplo, [7].
} 
vii) os pares $\left\{\Sigma, i d_{C}\right\}$ e $\left\{T, i d_{C}\right\}$ têm coequalizadores.

Observação 1.2.1. Eventualmente, o leitor pode querer, por questões de clareza, expressar condições, tais como a condição vi) acima, suprimindo a representação das projeções canônicas. Neste caso, a condição seria escrita como

$$
\mu\left(\mu, i d_{C}\right) \approx \mu\left(i d_{C}, \mu\right)
$$

A expressão anterior, apesar de esteticamente mais aprazível, é computacionalmente pouco informativa e será, sempre que possível, evitada. $\diamond$

Observação 1.2.2. Uma consequência importante da condição $i$ ), na definição acima, é que $\Sigma$ e $T$ são idempotentes. Para ver isto, basta notar que

$$
T T=T \Sigma T=\Sigma T=T
$$

A outra afirmação prova-se de maneira semelhante. Assim, as condições $i$ ) e vii) significam que $\Sigma$ e $T$ são idempotentes que cindem ${ }^{6}$. $\diamond$

Definição 1.2.2. Sejam $\mathbf{C}$ uma categoria, $(G, \Sigma, T, \mu)$ uma categoria em $\mathbf{C} e \iota \in \operatorname{End}_{\mathbf{C}}(G)$. Denote a identidade relativa a $G$ por $i d_{G}$. Dizemos que $(G, \Sigma, T, \mu, \iota)$ é um grupoide em $\mathbf{C}$, se estes dados satisfizerem:

i) $T \iota=\Sigma, \iota \Sigma=\Sigma e \iota \iota=i d_{G}$;

ii) $\mu\left(i d_{G}, \iota\right)_{\langle\Sigma, T\rangle}=T$ e $\mu\left(\iota, i d_{G}\right)_{\langle\Sigma, T\rangle}=\Sigma$.

A equivalência entre esta definição de categoria interna (e grupoide interno) e a usual é expressa nas seguintes proposições.

Proposição 1.2.1. Sejam $\mathbf{C}$ uma categoria com os limites necessários e $(C, \Sigma, T, \mu)$ uma categoria em C. Então, a afirmação de que $\left\{\Sigma, i d_{C}\right\}$ e $\left\{T, i d_{C}\right\}$ têm coequalizadores é equivalente a afirmar que $\left\{\Sigma, i d_{C}\right\}$ e $\left\{T, i d_{C}\right\}$ têm um equalizador comum.

Demonstração. Suponha que $\varepsilon: M \rightarrow C$ seja um equalizador comum para $\left\{\Sigma, i d_{C}\right\}$ e $\left\{T, i d_{C}\right\}$. Pela observação 1.2.2, $\Sigma$ é idempotente e, portanto, iguala $\Sigma$ e $i d_{C}$. Da propriedade universal de equalizadores, existe um único morfismo $\sigma: C \rightarrow M$, tal que $\Sigma=\varepsilon \sigma$. Afirmamos que $\sigma$ é coequalizador de $\Sigma$ e $i d_{C}$. Para ver isto, note primeiramente que $\sigma \varepsilon=i d_{M}$, pois

$$
\varepsilon \sigma \varepsilon=\Sigma \varepsilon=\varepsilon
$$

donde $\varepsilon \sigma \varepsilon$ iguala $\Sigma$ e $i d_{C}$. Da propriedade universal de equalizadores, $i d_{M}$ é o único morfismo tal que $\varepsilon i d_{M}=\varepsilon$. Logo, só pode ser $\sigma \varepsilon=i d_{M}$. Isso posto, temos que,

$$
\sigma \Sigma=\sigma \varepsilon \sigma=i d_{M} \sigma=\sigma
$$

mostrando que $\sigma$ coiguala $\Sigma$ e $i d_{C}$. Agora, considere $g: C \rightarrow A$, tal que $g \Sigma=g$. Defina $\bar{g}: M \rightarrow A$ por $\bar{g}=g \varepsilon$. Então,

$$
\bar{g} \sigma=g \varepsilon \sigma=g \Sigma=g
$$

Suponha que $\overline{\bar{g}}$ seja outro morfismo tal que $\overline{\bar{g}} \sigma=g$. Então,

$$
\begin{aligned}
& \overline{\bar{g}} \sigma=g=\bar{g} \sigma \\
& \overline{\bar{g}} \sigma \varepsilon=\bar{g} \sigma \varepsilon \\
& \overline{\bar{g}} i d_{M}=\bar{g} i d_{M} \\
& \overline{\bar{g}}=\bar{g}
\end{aligned}
$$

\footnotetext{
${ }^{6}$ Um morfismo $f$ é um idempotente que cinde, se $f^{2}=f$ e se existirem morfismos $g, h$, tais que $g h=f$ e $h g=1$.
} 
Assim, $\sigma$ é coequalizador do par $\left\{\Sigma, i d_{C}\right\}$. Um raciocínio análogo fornece um morfismo $\tau: C \rightarrow M$, coequalizador do par $\left\{T, i d_{C}\right\}$.

Por outro lado, suponha que $\sigma: C \rightarrow M$ seja coequalizador de $\left\{\Sigma, i d_{C}\right\}$ e $\tilde{\tau}: C \rightarrow N$ seja coequalizador de $\left\{T, i d_{C}\right\}$. Pela observação $1.2 .2, \Sigma$ é idempotente e, portanto, coiguala $\Sigma$ e $i d_{C}$. Da propriedade universal de coequalizadores, existe um único morfismo $\varepsilon: M \rightarrow C$, tal que $\varepsilon \sigma=\Sigma$. Afirmamos que $\varepsilon$ é equalizador de $\left\{\Sigma, i d_{C}\right\}$. Para ver isto, note primeiramente que

$$
\sigma \varepsilon \sigma=\sigma \Sigma=\sigma
$$

donde, da propriedade universal de coequalizadores para $\sigma$, segue que $\sigma \varepsilon=i d_{M}$. Agora,

$$
\Sigma \varepsilon=\varepsilon \sigma \varepsilon=\varepsilon i d_{M}=\varepsilon
$$

mostrando que $\varepsilon$ iguala $\Sigma$ e $i d_{C}$. Seja $h: B \rightarrow C$, tal que $\Sigma h=h$. Defina $\bar{h}: B \rightarrow M$ por $\bar{h}=\sigma h$. Assim,

$$
\varepsilon \bar{h}=\varepsilon \sigma h=\Sigma h=h
$$

Se $\overline{\bar{h}}$ for outro morfismo tal que $\varepsilon \overline{\bar{h}}=h$, temos

$$
\begin{aligned}
& \varepsilon \overline{\bar{h}}=h=\varepsilon \bar{h} \\
& \sigma \varepsilon \overline{\bar{h}}=\sigma \varepsilon \bar{h} \\
& i d_{M} \overline{\bar{h}}=i d_{M} \bar{h} \\
& \overline{\bar{h}}=\bar{h}
\end{aligned}
$$

que mostra que $\varepsilon$ é equalizador de $\Sigma$ e $i d_{C}$. Um raciocínio análogo fornece um morfismo $\tilde{\varepsilon}: N \rightarrow C$, equalizador de $T$ e $i d_{C}$, satisfazendo $\tilde{\varepsilon} \tilde{\tau}=T$ e $\tilde{\tau} \tilde{\varepsilon}=i d_{N}$. Afirmamos que $M$ e $N$ são isomorfos, com isomorfismos $\sigma \tilde{\varepsilon}$ e $\tilde{\tau} \varepsilon$, mutuamente inversos. Para ver isto, basta notar que

$$
\begin{aligned}
& \Sigma T=T \\
& \varepsilon \sigma \tilde{\varepsilon} \tilde{\tau}=\tilde{\varepsilon} \tilde{\tau} \\
& \varepsilon \sigma \tilde{\varepsilon}=\tilde{\varepsilon} \\
& \tilde{\tau} \varepsilon \sigma \tilde{\varepsilon}=i d_{N}
\end{aligned}
$$

Analogamente, de $T \Sigma=\Sigma$, segue que $\sigma \tilde{\varepsilon} \tilde{\tau} \varepsilon=i d_{M}$. É importante notar que $\varepsilon$ e $\tilde{\varepsilon}$ são monomorfismos, pois se $f$ e $g$ forem morfismos tais que $\varepsilon f=\varepsilon g$, então $\sigma \varepsilon f=\sigma \varepsilon g$, donde $f=g$. O mesmo para $\tilde{\varepsilon}$.

Definamos $\tau=\sigma \tilde{\varepsilon} \tilde{\tau}$. Temos então, $\tau: C \rightarrow M$, coequalizador de $\left\{T, i d_{C}\right\}$, com

$$
\begin{gathered}
\varepsilon \tau=\varepsilon \sigma \tilde{\varepsilon} \tilde{\tau}=\Sigma T=T \\
\tau \varepsilon=\sigma \tilde{\varepsilon} \tilde{\tau} \varepsilon=i d_{M}
\end{gathered}
$$

Portanto,

$$
T \varepsilon=\varepsilon \tau \varepsilon=\varepsilon
$$

Como $\varepsilon=\tilde{\varepsilon}(\sigma \tilde{\varepsilon})^{-1}=\tilde{\varepsilon} \tilde{\tau} \varepsilon$, temos que $\varepsilon$ é equalizador de $\left\{T, i d_{C}\right\}$.

Proposição 1.2.2. Sejam $\mathbf{C}$ uma categoria com os limites necessários e $(C, \Sigma, T, \mu)$ uma categoria em $\mathbf{C}$. A afirmação de que os pares $\left\{\Sigma, i d_{C}\right\}$ e $\left\{T, i d_{C}\right\}$ têm coequalizadores é equivalente a afirmar que existe um sub-objeto $M$ de $C$, ou seja, um monomorfismo $\varepsilon: M \rightarrow C$, tal que o diagrama

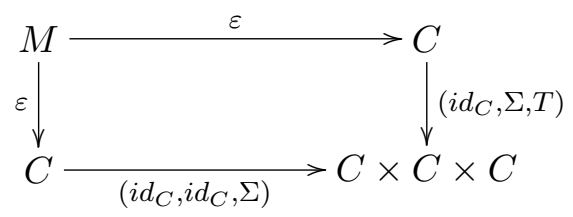


seja um diagrama de pull-back.

Demonstração. Da proposição anterior, podemos obter $\sigma: C \rightarrow M$ e $\tau: C \rightarrow M$, coequalizadores para $\left\{\Sigma, i d_{C}\right\}$ e $\left\{T, i d_{C}\right\}$, respectivamente, e $\varepsilon: M \rightarrow C$, um equalizador comum para $\left\{\Sigma, i d_{C}\right\}$ e $\left\{T, i d_{C}\right\}$, monomorfismo, de modo que $\sigma, \tau$ e $\varepsilon$ satisfaçam todas as condições de cisão para $\Sigma$ e $T$. Assim,

$$
T \varepsilon=\varepsilon=\Sigma \varepsilon
$$

que, aliada às condições anteriores, fornece o diagrama

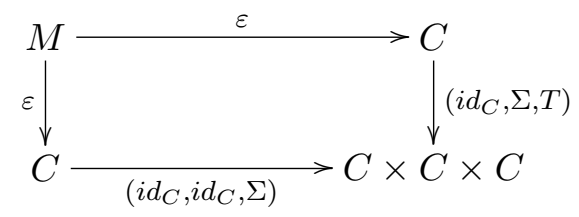

comutativo. Para mostrar que é um diagrama de pull-back, considere um cone $C \stackrel{\beta}{\leftarrow} D \stackrel{\alpha}{\rightarrow} C$, com $\left(i d_{C}, \Sigma, T\right) \alpha=\left(i d_{C}, i d_{C}, \Sigma\right) \beta$. Note que, portanto, $\alpha=\beta$. Definamos $\eta: D \rightarrow M$ por $\eta=\sigma \alpha$. Então,

$$
\varepsilon \eta=\varepsilon \sigma \alpha=\Sigma \alpha=\alpha
$$

Como $\varepsilon$ é monomorfismo, $\eta$ é o único morfismo com tal propriedade. Logo, o diagrama acima é um pull-back.

Por outro lado, suponha que o diagrama dado seja um pull-back. Mostremos que $\varepsilon$ é um equalizador comum para $\left\{\Sigma, i d_{C}\right\}$ e $\left\{T, i d_{C}\right\}$. De fato, temos que $\Sigma \varepsilon=\varepsilon$ e $T \varepsilon=\Sigma \varepsilon=\varepsilon$, mostrando que $\varepsilon$ iguala $\left\{\Sigma, i d_{C}\right\}$ e $\left\{T, i d_{C}\right\}$. Se $g: A \rightarrow C$ for um morfismo tal que $\Sigma g=g$, então

$$
T g=T \Sigma g=\Sigma g
$$

donde $C \stackrel{g}{\longleftarrow} A \stackrel{g}{\rightarrow} C$ é um cone tal que

$$
\left(i d_{C}, \Sigma, T\right) g=(g, \Sigma g, T g)=(g, g, \Sigma g)=\left(i d_{C}, i d_{C}, \Sigma\right) g
$$

Assim, da propriedade universal de pull-back, existe um único morfismo $\bar{g}: A \rightarrow M$, tal que $\varepsilon \bar{g}=g$, o que mostra que $\varepsilon$ é equalizador de $\left\{\Sigma, i d_{C}\right\}$. Se $h: B \rightarrow C$ for um morfismo tal que $T h=h$, então

$$
\Sigma h=\Sigma T h=T h=h
$$

donde $C \stackrel{h}{\leftarrow} B \stackrel{h}{\rightarrow} C$ é um cone tal que

$$
\left(i d_{C}, \Sigma, T\right) h=(h, \Sigma h, T h)=(h, h, \Sigma h)=\left(i d_{C}, i d_{C}, \Sigma\right) h
$$

Por um raciocínio análogo ao do caso anterior, $\varepsilon$ é equalizador de $\left\{T, i d_{C}\right\}$. Portanto, $\varepsilon$ é um equalizador comum para $\left\{\Sigma, i d_{C}\right\}$ e $\left\{T, i d_{C}\right\}$. Da proposição anterior, podemos construir $\sigma: C \rightarrow M$ e $\tau: C \rightarrow M$, coequalizadores para $\left\{\Sigma, i d_{C}\right\}$ e $\left\{T, i d_{C}\right\}$, respectivamente.

Observação 1.2.3. Da proposição anterior, segue que poderíamos ter definido o conceito de categoria interna como uma sêxtupla $(C, M, \sigma, \tau, \mu, \varepsilon)$, onde $C$ e $M$ são objetos de uma categoria $\mathbf{C}$, $\sigma, \tau: C \rightarrow M, \varepsilon: M \rightarrow C$ e $\mu: C{ }_{\sigma} \times_{\tau} C \rightarrow C$, satisfazendo condições pertinentes. Não é difícil ver que $C{ }_{\sigma} \times{ }_{\tau} C$ é canonicamente isomorfo a $C{ }_{\Sigma} \times_{T} C$. Em muitas categorias concretas, isto é, categorias nas quais os objetos possuem conjuntos subjacentes, estes pull-backs, bem como suas projeções, coincidem. A razão para adotarmos a primeira abordagem, ao invés da segunda, está no fato de que, em muitos casos importantes, os morfismos $\sigma$ e $\tau$ devem satisfazer algumas condições que dependem da categoria subjacente. Por exemplo, deve-se requerer, em categorias internas a Man, a categoria de aplicações suaves entre variedades diferenciáveis que, tanto $\sigma$ como $\tau$, sejam submersões sobrejetoras. Muitas vezes, não é claro quais condições deveríamos exigir para que es- 
tas aplicações tenham as propriedades esperadas. Com a abordagem adotada aqui, tais exigências são desnecessárias. As propriedades esperadas são, na realidade, demonstráveis. Faremos isto mais adiante para o caso de grupoides internos a Man, ou seja, grupoides de Lie. $\diamond$

Definição 1.2.3. Sejam $\left(C, \Sigma_{C}, T_{C}, \mu_{C}\right)$ e $\left(D, \Sigma_{D}, T_{D}, \mu_{D}\right)$ categorias internas a uma categoria $\mathbf{C}$. Um funtor (interno, covariante) de $C$ em $D$ é um morfismo $F: C \rightarrow D$, tal que torna comutativos os seguintes diagramas:
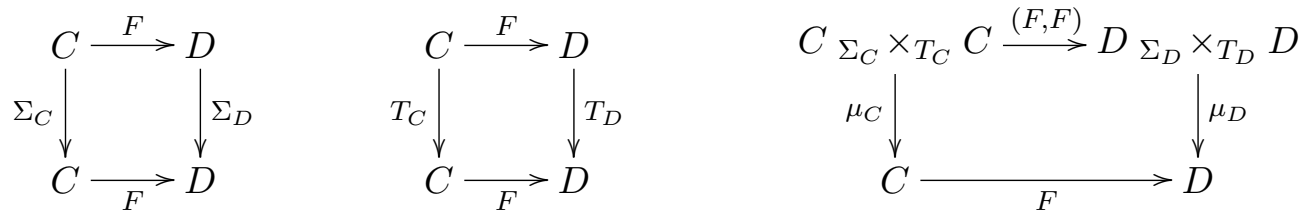

onde o morfismo $(F, F)$ no diagrama à direita é o único morfismo, representado abaixo pela seta tracejada, dado pelo diagrama

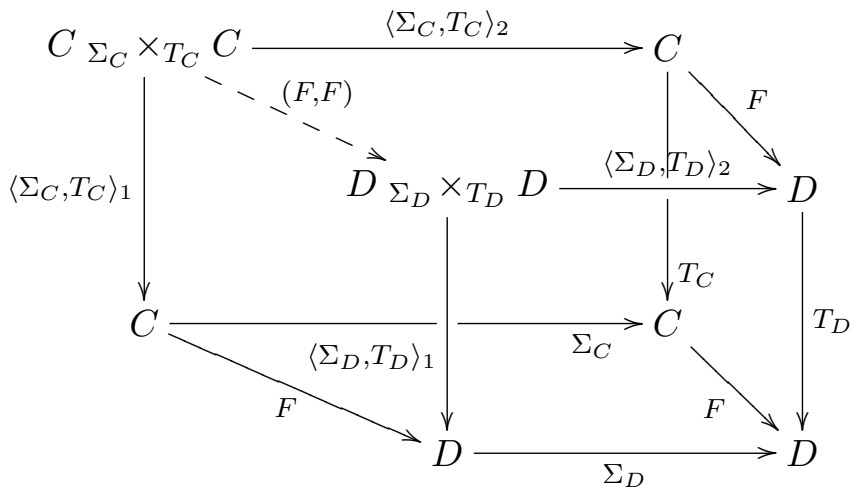

Definição 1.2.4. Dadas $\left(C, \Sigma_{C}, T_{C}, \mu_{C}\right)$ e $\left(D, \Sigma_{D}, T_{D}, \mu_{D}\right)$, categorias em $\mathbf{C}$, dizemos que $C$ é subcategoria de $D$, se existir um funtor $F: C \rightarrow D$, que seja um monomorfismo. Do mesmo modo, defini-se subgrupoide.

Observação 1.2.4. Usando a notação da definição anterior e denotando por $\varepsilon_{C}: M \rightarrow C$ e $\varepsilon_{D}: N \rightarrow D$ os morfismos identidade de $C$ e $D$, respectivamente, $F: C \rightarrow D$ um funtor significa que $F \varepsilon_{C}$ iguala o par $\left\{\Sigma_{D}, i d_{D}\right\}$. Disto, segue que existe um único morfismo $f: M \rightarrow N$, tal que $F \varepsilon_{C}=\varepsilon_{D} f$. O leitor pode provar com relativa facilidade que, se $F$ for monomorfismo, então $f$ também o será. Isto justifica a abordagem de subcategoria interna por monomorfismos aqui adotada. Caso, além disso, $f$ seja isomorfismo, dizemos que $C$ é subcategoria ampla de $D$.

Os conceitos de funtor pleno, fiel, sobrejetor em objetos, etc., dependem da lógica interna da categoria ambiente e não podem ser discutidos sem tal referência. ${ }^{7}$ Entretanto, tais conceitos têm sentido óbvio quando a categoria ambiente é concreta (usa-se um funtor para obter o conjunto subjacente e os conceitos da definição 1.1.4). $\diamond$

Definição 1.2.5. Sejam $\left(C, \Sigma_{C}, T_{C}, \mu_{C}\right)$ e $\left(D, \Sigma_{D}, T_{D}, \mu_{D}\right)$ categorias em $\mathbf{C}$ e $F, G: C \rightarrow D$ funtores em C. Uma transformação natural (em $\mathbf{C}$ ) de $F$ em $G$ é um morfismo $\alpha: C \rightarrow D$, que satisfaça

i) $\Sigma_{D} \alpha\left\langle\Sigma_{C}, T_{C}\right\rangle_{1}=T_{D} F\left\langle\Sigma_{C}, T_{C}\right\rangle_{2}$;

ii) $\Sigma_{D} G\left\langle\Sigma_{C}, T_{C}\right\rangle_{1}=T_{D} \alpha\left\langle\Sigma_{C}, T_{C}\right\rangle_{2}$;

iii) $\mu_{D}\left(\alpha\left\langle\Sigma_{C}, T_{C}\right\rangle_{1}, F\left\langle\Sigma_{C}, T_{C}\right\rangle_{2}\right)_{\left\langle\Sigma_{D}, T_{D}\right\rangle}=\alpha \mu_{C}=\mu_{D}\left(G\left\langle\Sigma_{C}, T_{C}\right\rangle_{1}, \alpha\left\langle\Sigma_{C}, T_{C}\right\rangle_{2}\right)_{\left\langle\Sigma_{D}, T_{D}\right\rangle}$.

\footnotetext{
${ }^{7}$ Uma sugestão para este tratamento é discutida na observação 1.2.16.
} 
Como nosso objetivo é descrever grupoides, e não apenas categorias, apresentaremos agora alguns exemplos com esta ênfase. Os detalhes destas construções podem ser encontrados nas notas [33].

Exemplo 1.2.1. Considere a categoria Man, constituída por aplicações infinitamente diferenciáveis entre variedades infinitamente diferenciáveis, localmente euclideanas, com topologia Hausdorff, cuja base seja enumerável. Uma categoria diferenciável, ou ainda, uma categoria de Lie é um objeto de categoria em Man. Um grupoide de Lie é um grupoide em Man.

Na literatura, muitas vezes o nome grupoide de Lie designa grupoides cuja "base" (o espaço de objetos) seja uma variedade suave, mas com um "espaço total" (o espaço de morfismos) um pouco mais geral, por exemplo, sem a exigência da topologia ser Hausdorff. São casos importantes, mas que aqui, receberão o nome de grupoides diferenciáveis, por uma questão de fecho conceitual (ou seja, gosto do autor).

Considere um grupoide $(G, \Sigma, T, \mu, \iota)$ em Man. Como $\Sigma$ e $T$ são idempotentes e têm mesma imagem em $G$, definem uma subvariedade mergulhada, denotada por $M$. Não é difícil perceber que tal mergulho é dado pelo morfismo $\varepsilon: M \rightarrow G$, descrito na proposição 1.2.2. Ainda destas propriedades, $\varepsilon$ é, na realidade, um mergulho próprio. As restrições de $\Sigma$ e $T$ às suas imagens definem os morfismos $\sigma$ e $\tau$, referidos na mesma proposição, que são, portanto, submersões sobrejetoras (dado que são restrições na imagem, a uma subvariedade mergulhada, de retrações). Note que a inversão $\iota$ é, necessariamente, um difeomorfismo. Na literatura, é comum encontrarmos a definição de grupoide de Lie como sendo a estrutura $(G, M, \sigma, \tau, \mu, \varepsilon, \iota)$, que satisfaça todas as propriedades listadas acima. Note que, em nossa abordagem, a "seção de unidades" $\varepsilon$ é uma consequência e não um requisito. $\diamond$

Exemplo 1.2.2. Seja $M$ uma variedade diferenciável. Denotemos por $\operatorname{Aut}_{l o c}^{\infty}(M)$ o espaço de todos os difeomorfismos locais entre abertos de $M$. Defina $\Sigma, T, \iota: \operatorname{Aut}_{l o c}^{\infty}(M) \rightarrow \operatorname{Aut}_{l o c}^{\infty}(M)$, dados por, para todo $f \in \operatorname{Aut}_{l o c}^{\infty}(M)$,

$$
\begin{aligned}
\Sigma(f) & =f^{-1} \circ f \\
T(f) & =f \circ f^{-1} \\
\iota(f) & =f^{-1}
\end{aligned}
$$

O produto $\mu$ é definido por $\mu(g, f)=g \circ f$, para $f, g \in \operatorname{Aut}_{l o c}^{\infty}(M)$, desde que $\Sigma(g)=T(f)$. A estrutura ( $\operatorname{Aut}_{l o c}^{\infty}(M), \Sigma, T, \mu, \iota$ ) é um exemplo de grupoide difeológico. O leitor pode encontrar definições relativas a espaços difeológicos (i.e., espaços com estrutura diferenciável generalizada), em [38]. Não nos será necessário entrar em detalhes sobre este assunto.

Podemos definir, do mesmo modo, o grupoide (topológico) Aut $_{l o c}^{0}(M)$, dos homeomorfismos entre abertos de $M$. Subgrupoides amplos de $\operatorname{Aut}_{l o c}^{0}(M)$ (ou seja, subgrupoides que contenham todas as identidades do grupoide ambiente) desempenham um papel fundamental na teoria das variedades diferenciáveis. Um sub-grupoide amplo $\Gamma \subseteq \operatorname{Aut}_{l o c}^{0}(M)$ que tenha a "propriedade de feixe", ou seja, dada uma cobertura aberta $U=\bigcup U_{\alpha}$ de um aberto $U$ de $M, f \in \Gamma$ se, e somente se, $f_{\alpha}=\left.f\right|_{\alpha} \in \Gamma$, para todo $\alpha$, é dito pseudogrupo de transformações de $M$. Tais pseudogrupos descrevem simetrias em variedades com alguma estrutura, podem ser usados para descrever estruturas adicionais em variedades, ou até mesmo podem ser usados para definirmos variedades diferenciáveis, como é feito, por exemplo, em [21]. $\diamond$

Exemplo 1.2.3. O leitor é convidado a mostrar que, dado um anel comutativo, com unidade, $R$ e denotando por RAss, LAlg e PAlg as categorias de homomorfismos entre $R$-álgebras associativas, de Lie e de Poisson, respectivamente, objetos de grupoide nestas categorias são exatamente as extensões abelianas em cada categoria, onde a álgebra base é estendida pelo espaço de morfismos. Em outras palavras, dada uma álgebra base, os objetos de grupoide sobre esta base são as estruturas de módulo, em cada categoria. $\diamond$ 
Exemplo 1.2.4. Sejam $\mathbf{C}$ uma categoria com objeto terminal 1 e $(G, \Sigma, T, \mu, \iota)$ um grupoide em $\mathbf{C}$. O grupoide anterior será um objeto de grupo, precisamente quando existir um elemento $e: 1 \rightarrow G$, tal que $\Sigma=T=e \circ !_{G}$, onde $!_{G}: G \rightarrow 1$ é o morfismo canônico e o é a composição em $\mathbf{C}$. $\diamond$

Observação 1.2.5. É interessante notar que podemos definir o conceito de cogrupoide em uma categoria simplesmente invertendo o sentido de todos os morfismos das estruturas. Ou seja, um cogrupoide em $\mathbf{C}$ é um grupoide em $\mathbf{C}^{o p}$. Cogrupoides em categorias de álgebras associativas são conhecidos na literatura como algebroides de Hopf. Quando em categorias de álgebras associativas, comutativas, com unidade, a situação dual à do exemplo anterior fornece exatamente as álgebras de Hopf. Nestes mesmos ambientes, podemos ver ainda que é possível obter uma descrição de algebroides de Hopf como quocientes de álgebras produto, dado que push-outs são dados por quocientes de coprodutos (e a álgebra produto, ou o produto tensorial de álgebras, é dada pelo coproduto nesta categoria). Isto simplifica bastante a descrição destes objetos. $\diamond$

Definição 1.2.6. Sejam $(C, \Sigma, T, \mu)$ uma categoria em $\mathbf{C}$ e $\varphi: E \rightarrow C$ um morfismo que iguale $\left\{\Sigma, i d_{C}\right\}$, ou seja, $\Sigma \varphi=\varphi$. Suponha que exista o objeto $C_{\Sigma} \times_{\varphi} E$. Uma ação (à esquerda) de $C$ em $\varphi$ (ou em $E$, quando não houver risco de confusão) é um morfismo $\Phi: C{ }_{\Sigma} \times_{\varphi} E \rightarrow E$, sob as seguintes condições:

i) torna comutativo o diagrama

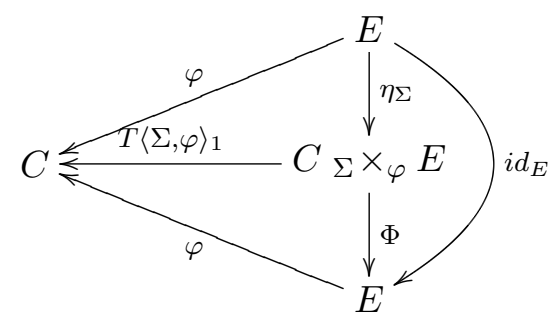

onde $\eta_{\Sigma}=\left(\varphi, i d_{E}\right)_{\langle\Sigma, \varphi\rangle}$

ii) existem os pull-backs $\left(C_{\Sigma} \times_{T} C\right)_{\Sigma\langle\Sigma, T\rangle_{2}} \times_{\varphi} E$ e $C_{\Sigma} \times_{T\langle\Sigma, \varphi\rangle_{1}}\left(C_{\Sigma} \times_{\varphi} E\right)$;

iii) é válida a igualdade, a menos de isomorfismo canônico,

$$
\begin{aligned}
\Phi \circ\left(\mu \circ\left\langle\Sigma\langle\Sigma, T\rangle_{2}, \varphi\right\rangle_{1},\left\langle\Sigma\langle\Sigma, T\rangle_{2}, \varphi\right\rangle_{2}\right)_{\langle\Sigma, \varphi\rangle} & \approx \\
& \approx \Phi \circ\left(\left\langle\Sigma, T\langle\Sigma, \varphi\rangle_{1}\right\rangle_{1}, \Phi \circ\left\langle\Sigma, T\langle\Sigma, \varphi\rangle_{1}\right\rangle_{2}\right)_{\langle\Sigma, \varphi\rangle}
\end{aligned}
$$

Observação 1.2.6. Caso o leitor sinta a necessidade de ignorar a representação das projeções canônicas, a condição $i i i$ ) na definição acima seria escrita como

$$
\Phi\left(\mu, i d_{E}\right) \approx \Phi\left(i d_{C}, \Phi\right)
$$

Preferiremos sempre a versão mais carregada, porém mais precisa. $\diamond$

Observação 1.2.7. Na literatura, o comum é encontrarmos a definição de ação de uma categoria ao longo de um morfismo, cujo codomínio seja o espaço de objetos da categoria, descrita em termos de objetos e morfismos. Tal descrição nos será útil. Tendo em mente as notações da definição anterior, denotemos por $(C, M, \sigma, \tau, \mu, \varepsilon)$ a mesma categoria, descrita por objetos e morfismos.

Note que, como $\varphi$ iguala $\left\{\Sigma, i d_{C}\right\}$, ao definirmos $f=\sigma \varphi$, temos que $C{ }_{\Sigma} \times{ }_{\varphi} E$ é canonicamente isomorfo a $C{ }_{\sigma} \times{ }_{f} E$. Um raciocínio análogo pode ser aplicado aos outros pull-backs envolvidos. Note, também, que $\varepsilon f=\varepsilon \sigma \varphi=\Sigma \varphi=\varphi$. Assim, o dicionário entre estas descrições, além das projeções envolvidas, se dá simplesmente por, dada $f$, faça $\varphi=\varepsilon f$; dada $\varphi$, faça $f=\sigma \varphi$. É importante perceber que, a menos de isomorfismo canônico (que, em categorias concretas, pode ser até mesmo uma igualdade), não há necessidade de se redefinir $\Phi$. $\diamond$ 
Observação 1.2.8. Outra observação importante é que a definição acima pode ser "refletida" para obtermos ações à direita. Basta que invertamos todas as condições que envolvam lateralidades, inclusive trocando-se os papeis de $\Sigma$ e $T$. Evidentemente, uma categoria age à esquerda ao longo de seu codomínio e à direita ao longo de seu domínio. $\diamond$

Observação 1.2.9. Caso $(C, \Sigma, T, \mu)$ seja um objeto de monoide em $\mathbf{C}$, ou seja, $\mathbf{C}$ tenha um objeto terminal 1 e exista $e: 1 \rightarrow C$, com $\Sigma=T=e \circ !_{C}$, temos que uma ação de $C$ em um morfismo $\varphi$ é o mesmo que uma ação de monoide, no sentido tradicional. Para ver isto, basta notar que o objeto base de $C$ é isomorfo a 1, donde podemos tomar $\sigma$ e $\tau$ como sendo os morfismos canônicos. Os pull-backs envolvidos se tornam produtos e $f=\sigma \varphi=!_{C} \varphi=!_{E}$. O mesmo vale para o caso de ações de grupos. $\diamond$

Proposição 1.2.3. Sejam $(C, \Sigma, T, \mu)$ uma categoria em $\mathbf{C}, \varphi: E \rightarrow C$ um morfismo que iguala $\left\{\Sigma, i d_{C}\right\}$ e $\Phi: C_{\Sigma} \times{ }_{\varphi} E \rightarrow E$ uma ação. Então, existe uma estrutura canônica de categoria, dita categoria de ação, denotada por $\left(C_{\Sigma} \times_{\varphi} E, \Sigma_{\Phi}, T_{\Phi}, \mu_{\Phi}\right)$, definida por:

i) $\Sigma_{\Phi}=\left(\Sigma\langle\Sigma, \varphi\rangle_{1},\langle\Sigma, \varphi\rangle_{2}\right)_{\langle\Sigma, \varphi\rangle}$

ii) $T_{\Phi}=\left(T\langle\Sigma, \varphi\rangle_{1}, \Phi\right)_{\langle\Sigma, \varphi\rangle}$

iii) $\mu_{\Phi}=\left(\mu \circ\left(\langle\Sigma, \varphi\rangle_{1} \circ\left\langle\Sigma_{\Phi}, T_{\Phi}\right\rangle_{1},\langle\Sigma, \varphi\rangle_{1} \circ\left\langle\Sigma_{\Phi}, T_{\Phi}\right\rangle_{2}\right)_{\langle\Sigma, T\rangle},\langle\Sigma, \varphi\rangle_{2} \circ\left\langle\Sigma_{\Phi}, T_{\Phi}\right\rangle_{2}\right)_{\langle\Sigma, \varphi\rangle}$

desde que exista o pull-back $\left(C_{\Sigma} \times_{\varphi} E\right)_{\Sigma_{\Phi}} \times_{T_{\Phi}}\left(C_{\Sigma} \times_{\varphi} E\right)$. Nas mesmas condições, se $(G, \Sigma, T, \mu, \iota)$ for um grupoide que aja sobre $\varphi$ com ação $\Phi$, como acima, temos definido um objeto de grupoide $\left(G_{\Sigma} \times_{\varphi} E, \Sigma_{\Phi}, T_{\Phi}, \mu_{\Phi}, \iota_{\Phi}\right)$, dito grupoide de ação, definido como acima, com o morfismo inversão dado por

$$
\iota_{\Phi}=\left(\iota \circ\langle\Sigma, \varphi\rangle_{1}, \Phi\right)_{\langle\Sigma, \varphi\rangle}
$$

Demonstração. Todas as verificações são cálculos diretos a partir das definições.

Observação 1.2.10. Quando descrevemos grupoides como $(G, M, \sigma, \tau, \mu, \varepsilon, \iota)$ e ações $\Phi: G{ }_{\sigma} \times{ }_{f} E \rightarrow E$, com $f: E \rightarrow M$ em termos de objetos e morfismos, o grupoide de ação, denotado por $\left(G_{\sigma} \times_{f} E, E, \sigma_{\Phi}, \tau_{\Phi}, \mu_{\Phi}, \varepsilon_{\Phi}, \iota_{\Phi}\right)$, é definido da seguinte maneira (note que a base é $E)$ :

i) $\sigma_{\Phi}=\langle\sigma, f\rangle_{2}$

ii) $\tau_{\Phi}=\Phi$

iii) $\mu_{\Phi}=\left(\mu \circ\left(\langle\sigma, f\rangle_{1} \circ\left\langle\sigma_{\Phi}, \tau_{\Phi}\right\rangle_{1},\langle\sigma, f\rangle_{1} \circ\left\langle\sigma_{\Phi}, \tau_{\Phi}\right\rangle_{2}\right)_{\langle\sigma, \tau\rangle},\langle\sigma, f\rangle_{2} \circ\left\langle\sigma_{\Phi}, \tau_{\Phi}\right\rangle_{2}\right)_{\langle\sigma, f\rangle}$

iv) $\varepsilon_{\Phi}=\left(\varepsilon f, i d_{E}\right)_{\langle\sigma, f\rangle}$

v) $\iota_{\Phi}=\left(\iota \circ\langle\sigma, f\rangle_{1}, \Phi\right)_{\langle\sigma, f\rangle}$

O mesmo pode ser dito quando tivermos apenas as estruturas de categoria (obviamente, sem a inversão). Usaremos esta descrição posteriormente. $\diamond$

Observação 1.2.11. Note que, em um grupoide, a inversão fornece um isomorfismo entre as construções de grupoide de ação à direita e à esquerda. O mesmo não pode ser dito de ações de categorias. A existência de uma ação à esquerda não implica a existência de uma ação à direita, no caso geral de categorias. $\diamond$

Internamente a uma categoria, podemos considerar a categoria dos funtores internos entre objetos de categoria (ou mesmo grupoides). Transformações naturais podem ser vistas como morfismos entre funtores (com duas possíveis composições, uma "horizontal" e outra "vertical"). Isto fornece uma estrutura de 2-categoria, na qual podemos considerar o conceito de isomorfismo entre objetos e o de equivalência entre objetos. Ao lidarmos com diagramas limite, como produtos, pull-backs, etc., devemos ter isto em mente. Para mais detalhes, o leitor poderá consultar [30], [19] e [6]. 
Observação 1.2.12. Suponha que $\left(C, \Sigma_{C}, T_{C}, \mu_{C}\right)$ e $\left(D, \Sigma_{D}, T_{D}, \mu_{D}\right)$ sejam objetos de categoria em $\mathbf{C}$ e que exista o produto $C \stackrel{\langle C, D\rangle_{1}}{\longleftarrow} C \times D \stackrel{\langle C, D\rangle_{2}}{\longrightarrow} D$. Se denotarmos as unidades de $C, D$ e $C \times D$ por $\varepsilon_{C}: M \rightarrow C, \varepsilon_{D}: N \rightarrow D$ e $\varepsilon_{(C, D)}: R \rightarrow C \times D$, respectivamente, verifica-se facilmente que $M \stackrel{\sigma_{C}\langle C, D\rangle_{1} \varepsilon_{(C, D)}}{\longleftarrow} \stackrel{\sigma_{D}\langle C, D\rangle_{2} \varepsilon_{(C, D)}}{\longrightarrow} N$ é diagrama produto de $M$ e $N$.

Quando temos categorias (ou grupoides) sobre a mesma base, é possível considerar a existência de um "produto fibrado" destas. A definição seguinte versa sobre grupoides, mas, obviamente, é possível para categorias. Basta que suprimamos a inversão.

Definição 1.2.7. Sejam $\left(G_{1}, M, \sigma_{1}, \tau_{1}, \mu_{1}, \varepsilon_{1}, \iota_{1}\right)$ e $\left(G_{2}, M, \sigma_{2}, \tau_{2}, \mu_{2}, \varepsilon_{2}, \iota_{2}\right)$ grupoides em $\mathbf{C}$, sobre a mesma base $M$. Caso exista, por produto fibrado de $G_{1}$ e $G_{2}$, entendemos o grupoide construído da seguinte maneira. Defina as âncoras $\alpha_{i}: G_{i} \rightarrow M \times M$, por $\alpha_{i}=\left(\tau_{i}, \sigma_{i}\right)$. O espaço total é dado pelo pull-back

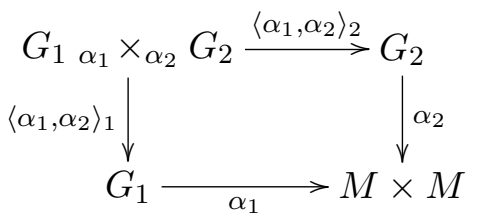

Os morfismos domínio e codominio são dados, respectivamente, por

$$
\begin{gathered}
\sigma_{12}=\sigma_{1}\left\langle\alpha_{1}, \alpha_{2}\right\rangle_{1}=\sigma_{2}\left\langle\alpha_{1}, \alpha_{2}\right\rangle_{2} \\
\tau_{12}=\tau_{1}\left\langle\alpha_{1}, \alpha_{2}\right\rangle_{1}=\tau_{2}\left\langle\alpha_{1}, \alpha_{2}\right\rangle_{2}
\end{gathered}
$$

A composta $\mu_{12}:\left(G_{1} \alpha_{1} \times_{\alpha_{2}} G_{2}\right)_{\sigma_{12}} \times_{\tau_{12}}\left(G_{1 \alpha_{1}} \times_{\alpha_{2}} G_{2}\right) \rightarrow G_{1 \alpha_{1}} \times_{\alpha_{2}} G_{2}$ é dada por

$$
\begin{aligned}
\mu_{12}=\left(\mu_{1}\left(\left\langle\alpha_{1}, \alpha_{2}\right\rangle_{1}\left\langle\sigma_{12}, \tau_{12}\right\rangle_{1},\left\langle\alpha_{1}, \alpha_{2}\right\rangle_{1}\left\langle\sigma_{12}, \tau_{12}\right\rangle_{2}\right)_{\left\langle\sigma_{1}, \tau_{1}\right\rangle},\right. & \\
& \left.\mu_{2}\left(\left\langle\alpha_{1}, \alpha_{2}\right\rangle_{2}\left\langle\sigma_{12}, \tau_{12}\right\rangle_{1},\left\langle\alpha_{1}, \alpha_{2}\right\rangle_{2}\left\langle\sigma_{12}, \tau_{12}\right\rangle_{2}\right)_{\left\langle\sigma_{2}, \tau_{2}\right\rangle}\right)_{\left\langle\alpha_{1}, \alpha_{2}\right\rangle}
\end{aligned}
$$

A seção de unidades é dada por

$$
\varepsilon_{12}=\left(\varepsilon_{1}, \varepsilon_{2}\right)_{\left\langle\alpha_{1}, \alpha_{2}\right\rangle}
$$

Finalmente, a inversão é dada por

$$
\iota_{12}=\left(\iota_{1}\left\langle\alpha_{1}, \alpha_{2}\right\rangle_{1}, \iota_{2}\left\langle\alpha_{1}, \alpha_{2}\right\rangle_{2}\right)_{\left\langle\alpha_{1}, \alpha_{2}\right\rangle}
$$

O grupoide referido é então $\left(G_{1 \alpha_{1}} \times_{\alpha_{2}} G_{2}, M, \sigma_{12}, \tau_{12}, \mu_{12}, \varepsilon_{12}, \iota_{12}\right)$.

Se $C$ for um objeto de categoria em Set, podemos extrair dos conjuntos de morfismos apenas aqueles que são isomorfismos, obtendo assim um grupoide, que é subcategoria de $C$. Além disso, ele é o "maior" subgrupoide de $C$. Isto nos leva à seguinte definição.

Definição 1.2.8. Seja $C$ um objeto de categoria em $\mathbf{C}$. Caso exista, dizemos que $G$ é subgrupoide máximo de $C$ quando $G$ for objeto de grupoide em $\mathbf{C}$ e existir um funtor $\imath: G \rightarrow C$, com $\imath$ monomorfismo em $\mathbf{C}$, satisfazendo a seguinte propriedade universal: se $H$ for um objeto de grupoide em $\mathbf{C}$, subgrupoide de $C$ dado, digamos, pelo monomorfismo J: $H \rightarrow C$, existe um único morfismo (de grupoides) $\bar{\jmath}: H \rightarrow G$, tal que $\jmath=\imath \circ \bar{\jmath}$.

Observação 1.2.13. Quando a categoria ambiente é concreta, a imagem de um objeto de categoria $C$ sempre possui um subgrupoide máximo. A questão então passa a ser se este subgrupoide pode ser "refletido" à categoria ambiente, ou seja, se o subgrupoide máximo obtido é imagem de algum grupoide na categoria ambiente e ainda, se a propriedade universal também se reflete. $\diamond$

Uma categoria interna possui sempre um monoide global externo a ela associada. ${ }^{8}$ Tal monoide,

\footnotetext{
${ }^{8}$ Aqui, na verdade, tacitamente supomos que a categoria ambiente possui suficientes características conjuntistas. Sem isto, não seria possível escrever a expressão $b \in \operatorname{Bis}(C)$, por exemplo. Isto é relevante nos casos em que se trabalha com categorias internas a categorias internas.
} 
por vezes, pode ser útil na análise de suas propriedades gerais. Precisemos este conceito.

Definição 1.2.9. Seja $(C, \Sigma, T, \mu)$ uma categoria em $\mathbf{C}$. Denote por $\varepsilon: M \rightarrow C$ o equalizador de identidades de $C$. Uma $\Sigma$-bisseção, ou simplesmente, uma bisseção é um morfismo $b: M \rightarrow C$, tal que $\Sigma b=\varepsilon$ e $T b=\varepsilon \varphi$, para algum $\varphi \in \operatorname{Aut}(M)$. A coleção das bisseções de $C$ (quando isto fizer sentido) será denotada por $\operatorname{Bis}(C)$.

Observação 1.2.14. Poderíamos ter definido o conceito de bisseção como T-bisseção, trocando os papeis de domínio e codomínio, ou ainda, deixando livre para que bisseções tenham isomorfismos em ambos os "lados", não apenas no domínio ou no codomínio. É fácil ver que a transição entre estas descrições é trivial. Entretanto, é mais fácil fixar uma escolha e trabalhar, sempre que possível, com esta. Note ainda que $C$ tem ao menos uma $\Sigma$-bisseção, a saber, $\varepsilon$. $\diamond$

Observação 1.2.15. As condições para que um morfismo $b: M \rightarrow C$ seja uma bisseção são equivalentes a se exigir que $\sigma b=i d_{M}$ e $\tau b=\varphi$, $\operatorname{com} \varphi \in \operatorname{Aut}(M)$. De fato, de $\Sigma=\varepsilon \sigma$, temos que a primeira condição se escreve como $\varepsilon \sigma b=\varepsilon$. Sendo $\varepsilon$ um monomorfismo, só pode ser $\sigma b=i d_{M}$. Analogamente, de $T=\varepsilon \tau$, temos $\varepsilon \tau b=\varepsilon \varphi$. Novamente do fato de ser $\varepsilon$ monomorfismo, segue que $\tau b=\varphi . \diamond$

Proposição 1.2.4. Suponha que $\mathbf{C}$ tenha os limites pertinentes. Seja $(C, \Sigma, T, \mu)$ uma categoria em $\mathbf{C}$. Então, podemos definir um monoide $(\operatorname{Bis}(C), m, \varepsilon)$. Seja $(G, \Sigma, T, \mu, \iota)$ um grupoide em $\mathbf{C}$. Então, podemos definir um grupo $(\operatorname{Bis}(G), m, \varepsilon, I)$.

Demonstração. A diferença entre $C$ e $G$ é apenas a existência da inversão. Assim, na definição de $m$, apenas troque $C$ por $G$, quando necessário. Definamos $m$ : $\operatorname{Bis}(C) \times \operatorname{Bis}(C) \rightarrow \operatorname{Bis}(C)$, por

$$
m\left(b, b^{\prime}\right)=\mu\left(b \tau b^{\prime}, b^{\prime}\right)_{\langle\sigma, \tau\rangle}
$$

para todas $b, b^{\prime} \in \operatorname{Bis}(C)$. Para ver que $m\left(b, b^{\prime}\right)$ é bisseção, basta fazermos

$$
\Sigma m\left(b, b^{\prime}\right)=\Sigma \mu\left(b \tau b^{\prime}, b^{\prime}\right)_{\langle\sigma, \tau\rangle}=\Sigma b^{\prime}=\varepsilon
$$

$\mathrm{e}$

$$
T m\left(b, b^{\prime}\right)=T \mu\left(b \tau b^{\prime}, b^{\prime}\right)_{\langle\sigma, \tau\rangle}=T b \tau b^{\prime}=\varepsilon \varphi \tau \varepsilon \tau b^{\prime}=\varepsilon \varphi \tau T b^{\prime}=\varepsilon \varphi \tau \varepsilon \varphi^{\prime}=\varepsilon \varphi \varphi^{\prime}
$$

notando ainda que $\varphi \varphi^{\prime} \in \operatorname{Aut}(M)$. Para mostrar a associatividade de $m$, tomemos $b, b^{\prime}, b^{\prime \prime} \in \operatorname{Bis}(C)$. Então, por um lado,

$$
m\left(m\left(b, b^{\prime}\right), b^{\prime \prime}\right)=\mu\left(\mu\left(b \tau b^{\prime}, b^{\prime}\right)_{\langle\sigma, \tau\rangle} \tau b^{\prime \prime}, b^{\prime \prime}\right)_{\langle\sigma, \tau\rangle}
$$

Por outro lado,

$$
m\left(b, m\left(b^{\prime}, b^{\prime \prime}\right)\right)=\mu\left(b \tau \mu\left(b^{\prime} \tau b^{\prime \prime}, b^{\prime \prime}\right)_{\langle\sigma, \tau\rangle}, \mu\left(b^{\prime} \tau b^{\prime \prime}, b^{\prime \prime}\right)_{\langle\sigma, \tau\rangle}\right)_{\langle\sigma, \tau\rangle}
$$

Definamos agora os morfismos

$$
\left(\left(b \tau b^{\prime}, b^{\prime}\right)_{\langle\sigma, \tau\rangle} \tau b^{\prime \prime}, b^{\prime \prime}\right)_{\left\langle\sigma\langle\sigma, \tau\rangle_{2}, \tau\right\rangle}: M \rightarrow\left(C_{\sigma} \times_{\tau} C\right)_{\sigma\langle\sigma, \tau\rangle_{2}} \times_{\tau} C
$$

$\mathrm{e}$

$$
\left(b \tau \mu\left(b^{\prime} \tau b^{\prime \prime}, b^{\prime \prime}\right)_{\langle\sigma, \tau\rangle},\left(b^{\prime} \tau b^{\prime \prime}, b^{\prime \prime}\right)_{\langle\sigma, \tau\rangle}\right)_{\left\langle\sigma, \tau\langle\sigma, \tau\rangle_{1}\right\rangle}: M \rightarrow C_{\sigma} \times_{\tau\langle\sigma, \tau\rangle_{1}}\left(C_{\sigma} \times_{\tau} C\right)
$$

Note que estes morfismos são iguais, a menos do isomorfismo canônico entre $\left(C{ }_{\sigma} \times{ }_{\tau} C\right)_{\sigma\langle\sigma, \tau\rangle_{2}} \times{ }_{\tau} C$ e $C{ }_{\sigma} \times_{\tau\langle\sigma, \tau\rangle_{1}}\left(C{ }_{\sigma} \times{ }_{\tau} C\right)$, devido ao fato de que ambos podem ser expressos como $\left(b \tau b^{\prime} \tau b^{\prime \prime}, b^{\prime} \tau b^{\prime \prime}, b^{\prime \prime}\right): M \rightarrow C_{\sigma} \times_{\tau} C_{\sigma} \times_{\tau} C$, dado que $b \tau \mu\left(b^{\prime} \tau b^{\prime \prime}, b^{\prime \prime}\right)=b \tau b^{\prime} \tau b^{\prime \prime}$. A associatividade (a menos de isomorfismo canônico) de $m$ seguirá ao aplicarmos a associatividade de $\mu$ nos morfismos previamente definidos, que fornecem exatamente as equações 1.1 e 1.2.

Que $\varepsilon$ é elemento neutro, é trivial. Assim, $(\operatorname{Bis}(C), m, \varepsilon)$ é monoide. Consideremos agora o caso de grupoides. Definamos $I: \operatorname{Bis}(G) \rightarrow \operatorname{Bis}(G)$, por

$$
I(b)=\iota b(\tau b)^{-1}
$$


para toda $b \in \operatorname{Bis}(G)$. Temos que $I(b) \in \operatorname{Bis}(G)$, pois

$$
\Sigma I(b)=\Sigma \iota b(\tau b)^{-1}=T b(\tau b)^{-1}=\varepsilon
$$

e ainda

$$
T I(b)=T \iota b(\tau b)^{-1}=\Sigma b(\tau b)^{-1}=\varepsilon(\tau b)^{-1}
$$

notando que $(\tau b)^{-1} \in \operatorname{Aut}(M)$. Para ver que $I$ é inversão, façamos

$$
m(b, I(b))=\mu\left(b \tau \iota b(\tau b)^{-1}, \iota b(\tau b)^{-1}\right)_{\langle\sigma, \tau\rangle}=\mu(b, \iota b)_{\langle\sigma, \tau\rangle}(\tau b)^{-1}=T b(\tau b)^{-1}=\varepsilon
$$

e também

$$
m(I(b), b)=\mu\left(\iota b(\tau b)^{-1} \tau b, b\right)_{\langle\sigma, \tau\rangle}=\mu(\iota b, b)_{\langle\sigma, \tau\rangle}=\Sigma b=\varepsilon
$$

mostrando que $(\operatorname{Bis}(G), m, \varepsilon, \iota)$ é grupo.

Assim, nas condições anteriores, podemos nos referir ao monoide de bisseções de uma categoria $(C, \Sigma, T, \mu)$ ou ao grupo de bisseções de um grupoide $(G, \Sigma, T, \mu, \iota)$ como as construções de $(\operatorname{Bis}(C), m, \varepsilon)$ e $(\operatorname{Bis}(G), m, \varepsilon, I)$, respectivamente, elaboradas na proposição anterior.

Proposição 1.2.5. Seja $\Phi: G_{\sigma \times} E \rightarrow E$ uma ação do grupoide $(G, M, \sigma, \tau, \mu, \varepsilon, \iota)$ em $f: E \rightarrow M$. Então, existe um homomorfismo de grupos de $\operatorname{Bis}(G)$ para $\operatorname{Bis}\left(G{ }_{\sigma} \times_{f} E\right)$. Se $f$ for epimorfismo, $\operatorname{Bis}(G)$ é subgrupo de $\operatorname{Bis}\left(G_{\sigma} \times_{f} E\right)$.

Demonstração. Tomemos $b \in \operatorname{Bis}(G)$. Façamos

$$
B=\left(b f, i d_{E}\right)_{\langle\sigma, f\rangle}
$$

que faz sentido, pois $\sigma b f=f$. Obviamente, $B$ é seção de $\sigma_{\Phi}$, pois

$$
\sigma_{\Phi} B=\langle\sigma, f\rangle_{2}\left(b f, i d_{E}\right)_{\langle\sigma, f\rangle}=i d_{E}
$$

Para mostrar que $B$ é bisseção de $G_{\sigma} \times_{f} E$, basta mostrar que $\tau_{\Phi} B$, ou seja, $\Phi B$, é automorfismo de $E$. Faremos isto construindo uma inversa para $\Phi B$. Tomemos

$$
\tilde{B}=\left(I(b) f, i d_{E}\right)_{\langle\sigma, f\rangle}=\left(\iota b(\tau b)^{-1} f, i d_{E}\right)_{\langle\sigma, f\rangle}
$$

Então,

$$
\begin{aligned}
\Phi B \Phi \tilde{B} & =\Phi\left(b f, i d_{E}\right) \Phi\left(\iota b(\tau b)^{-1} f, i d_{E}\right)= \\
& =\Phi\left(b f \Phi\left(\iota b(\tau b)^{-1} f, i d_{E}\right), \Phi\left(\iota b(\tau b)^{-1} f, i d_{E}\right)\right)= \\
& =\Phi\left(b(\tau b)^{-1} f, \Phi\left(\iota b(\tau b)^{-1} f, i d_{E}\right)\right)= \\
& =\Phi\left(\mu\left(b(\tau b)^{-1} f, \iota b(\tau b)^{-1} f\right), i d_{E}\right)= \\
& =\Phi\left(\varepsilon \tau b(\tau b)^{-1} f, i d_{E}\right)=\Phi\left(\varepsilon f, i d_{E}\right)= \\
& =i d_{E}
\end{aligned}
$$

Por outro lado,

$$
\begin{aligned}
\Phi \tilde{B} \Phi B & =\Phi\left(\iota b(\tau b)^{-1} f, i d_{E}\right) \Phi\left(b f, i d_{E}\right)= \\
& =\Phi\left(\iota b(\tau b)^{-1} f \Phi\left(b f, i d_{E}\right), \Phi\left(b f, i d_{E}\right)\right)= \\
& =\Phi\left(\iota b f, \Phi\left(b f, i d_{E}\right)\right)=\Phi\left(\mu(\iota b f, b f), i d_{E}\right)= \\
& =\Phi\left(\varepsilon \sigma b f, i d_{E}\right)=\Phi\left(\varepsilon f, i d_{E}\right)= \\
& =i d_{E}
\end{aligned}
$$


o que mostra, simultaneamente, que $B$ e $\tilde{B}$ são bisseções de $G_{\sigma} \times_{f} E$. Além disso,

$$
\begin{aligned}
I(B) & =\iota_{\Phi} B(\Phi B)^{-1}=\left(\iota\langle\sigma, f\rangle_{1}, \Phi\right)\left(b f, i d_{E}\right)(\Phi B)^{-1}=\left(\iota b f(\Phi B)^{-1}, i d_{E}\right)= \\
& =\left(\iota b f \Phi \tilde{B}, i d_{E}\right)=\left(\iota b \tau I(b) f, i d_{E}\right)=\left(\iota b(\tau b)^{-1} f, i d_{E}\right)= \\
& =\tilde{B}
\end{aligned}
$$

Agora, consideremos $b^{\prime}, b \in \operatorname{Bis}(G)$ e façamos $B^{\prime}=\left(b^{\prime} f, i d_{E}\right), B=\left(b f, i d_{E}\right)$. Assim,

$$
\begin{aligned}
m_{\Phi}\left(B^{\prime}, B\right) & =\mu_{\Phi}\left(B^{\prime} \Phi B, B\right)=\mu_{\Phi}\left(\left(b^{\prime} f, i d_{E}\right) \Phi\left(b f, i d_{E}\right),\left(b f, i d_{E}\right)\right)= \\
& =\mu_{\Phi}\left(\left(b^{\prime} \tau b f, \Phi\left(b f, i d_{E}\right)\right),\left(b f, i d_{E}\right)\right)=\left(\mu\left(b^{\prime} \tau b, b\right) f, i d_{E}\right)= \\
& =\left(m\left(b^{\prime}, b\right) f, i d_{E}\right)
\end{aligned}
$$

que estabelece o homomorfismo de grupos desejado. Suponha agora que $f$ seja epimorfismo. De $\left(b^{\prime} f, i d_{E}\right)_{\langle\sigma, f\rangle}=\left(b f, i d_{E}\right)_{\langle\sigma, f\rangle}$, é imediato que $b^{\prime} f=b f$ e, portanto, $b^{\prime}=b$.

Observação 1.2.16. Em muitos casos, a categoria ambiente vem munida de um conceito de localização. Por exemplo, em categorias internas a Top, a categoria das aplicações contínuas entre espaços topológicos, existe a possibilidade de estabelecermos condições ou relações entre espaços topológicos, com suas coberturas abertas, e as estruturas envolvidas. Faz sentido, por exemplo, falarmos em bisseções locais de uma tal categoria. É possível obter uma noção geral de bisseções locais em uma categoria interna, nos casos em que a categoria ambiente seja um "site", ou seja, uma categoria com uma "boa noção" de cobertura. Não faremos isto aqui, restringindo nosso tratamento a casos específicos, como Top e Man, evitando complicações desnecessárias. Isto será objeto de análise no próximo capítulo. O leitor interessado pode encontrar uma boa explanação sobre sites em [31] e sobre a internalização de conceitos em sites, com técnicas apropriadas de localização, em [35]. $\diamond$

Observação 1.2.17. Suponha que $C$ seja uma categoria concreta $^{9}$, de modo que possamos falar em conjuntos de objetos e conjuntos de morfismos entre objetos de $C$. Para cada objeto $x \in C$, a composição $\mu$ em $C$, restrita a $\operatorname{Hom}_{C}(x, x)$, faz de $\left(\operatorname{Hom}_{C}(x, x), \mu, i d_{x}\right)$, um monoide. Dado um outro objeto $y \in C$, se $\operatorname{Hom}_{C}(x, y)$ não for vazio, a composição $\mu$ fornece, por restrição, as ações $\mu: \operatorname{Hom}_{C}(x, y) \times \operatorname{Hom}_{C}(x, x) \rightarrow \operatorname{Hom}_{C}(x, y)$ e $\mu: \operatorname{Hom}_{C}(y, y) \times \operatorname{Hom}_{C}(x, y) \rightarrow \operatorname{Hom}_{C}(x, y)$. Deste modo, grosseiramente falando, uma categoria concreta pode ser vista como uma reunião disjunta de monoides, os monoides de "vértices" $\operatorname{Hom}_{C}(x, x)$, e espaços sobre os quais estes monoides agem à direita e à esquerda, os conjuntos "hom" $\operatorname{Hom}_{C}(x, y)$. Assim, os morfismos com domínio e codomínio diferentes servem como pontes, como ligações, entre os monoides de vértices. Ainda, se existir algum $f \in \operatorname{Hom}_{C}(x, y)$, que seja inversível, as operações de conjugação (composição por um lado com $f$ e por outro com $f^{-1}$ ) fornecem isomorfismos entre $\operatorname{Hom}_{C}(x, x)$ e $\operatorname{Hom}_{C}(y, y)$. Ou seja, um isomorfismo entre $x$ e $y$ torna também isomorfos os monoides $\operatorname{Hom}_{C}(x, x)$ e $\operatorname{Hom}_{C}(y, y)$. No caso de um grupoide, digamos $G$, no qual todo morfismo é isomorfismo, cada $\operatorname{Hom}_{G}(x, x)$ é grupo e cada $\operatorname{Hom}_{G}(x, y)$, que não seja vazio, estabelece isomorfismos entre os grupos de vértices no domínio e no codomínio. Assim, um grupoide concreto pode ser visto como uma reunião disjunta de grupos, que pode ser particionada em setores de grupos isomorfos, pela existência ou inexistência de morfismos entre eles. Em outras palavras, os conjuntos $\operatorname{Hom}_{C}(x, y)$ servem como espaços sobre os quais agem os grupos de vértices, listam todos os isomorfismos entre $x$ e $y$ e particionam o conjunto de grupos de vértices. Ao lidar com grupoides internos a categorias concretas, como grupoides e Lie, por exemplo, esta será uma imagem útil de se ter em mente. $\diamond$

A ideia de usarmos grupoides de Lie para descrever simetrias em teoria clássica dos campos tem forte motivação na proposição acima e no parágrafo anterior. A cada ponto de uma "base" $M$, geralmente pensada como o espaço-tempo, associa-se um grupo de vértice (que é um grupo de Lie), que descreve simetrias internas àquele ponto. O parágrafo anterior versa, portanto, sobre

\footnotetext{
${ }^{9}$ Note que $C$ pode ser uma categoria concreta, interna a uma categoria $\mathbf{C}$.
} 
a estrutura local, ou mesmo pontual, que um grupoide pode modelar, bem como determinadas aplicações de um ponto da base a outro. Já a proposição 1.2.4, versa sobre a estrutura global que um grupoide pode modelar, listando quais automorfismos de $M$ fazem parte da estrutura selecionada. A consequência imediata disto é a possibilidade de uma descrição conjunta de simetrias internas e simetrias na base, necessária para descrevermos leis de conservação com uma linguagem unificada. É interessante notar, ainda, que a passagem entre estas duas construções é uma ponte entre o tratamento de simetrias em dimensão infinita, dadas pelo grupo de bisseções, e o tratamento em dimensão finita, dadas pela estrutura local do grupoide. As considerações específicas sobre grupoides internos a Man serão objeto de análise no próximo capítulo. 


\section{Capítulo 2}

\section{Categorias e grupoides internos à categoria de variedades suaves}

\subsection{Grupoides de Lie e estruturas derivadas}

Como mencionado no exemplo 1.2.1, grupoides de Lie são grupoides internos a Man, a categoria das aplicações suaves entre variedades suaves. Por questões de comodidade e deferência às construções amplamente divulgadas na literatura existente, usaremos as versões com objeto de identidades explícito, ou seja, uma categoria interna a Man será denotada como em $(C, M, \sigma, \tau, \mu, \varepsilon)$. Além da já mencionada compatibilidade, esta notação explicita a "base" $M$, que futuramente desempenhará o papel de espaço-tempo, tornando as construções mais tangíveis à teoria dos campos, alvo desta tese. A principal referência para conceitos básicos em grupoides de Lie será [29].

A primeira observação a ser feita, apesar de evidente, é que grupoides de Lie (e categorias internas a Man, em geral) são categorias concretas. Isto permite que falemos sobre conjuntos de morfismos entre objetos, grupos (ou monoides) de vértices, grupos (ou monoides) de bisseções, etc. A proposição a seguir será apresentada sem demonstração, que poderá ser encontrada na referência supracitada (com uma leve adaptação).

Proposição 2.1.1. Seja $(G, M, \sigma, \tau, \mu, \varepsilon, \iota)$ um grupoide de Lie. Para cada objeto $x \in M$, o grupo de vértice $\operatorname{Hom}_{G}(x, x)$ é um grupo de Lie.

Assim, de acordo com a observação 1.2.17, grupoides de Lie podem ser vistos como coleções de fibrados de grupos de Lie, onde a existência de um morfismo interno entre estes grupos, ou seja, um isomorfismo de grupos listado entre os morfismos do grupoide, define uma relação entre estas fibras que fornece uma partição de $M$. Em cada classe desta partição, para cada dois objetos dados, há sempre um morfismo entre eles. Dentro da teoria de grupoides de Lie, esta propriedade merece atenção especial.

Definição 2.1.1. Diremos que um grupoide de Lie é transitivo quando, para cada par de objetos, existir ao menos um morfismo entre eles.

Alguns exemplos que nos serão úteis mais tarde.

Exemplo 2.1.1. Não é difícil para o leitor verificar que o seguinte grupoide é transitivo. Seja $M$ uma variedade diferenciável. Defina o grupoide de pares $\left(M \times M, \sigma_{M}, \tau_{M}, \mu_{M}, \varepsilon_{M}, \iota_{M}\right)$, com $\sigma_{M}=\langle M, M\rangle_{2}, \tau_{M}=\langle M, M\rangle_{1}, \mu_{M}: M\langle M, M\rangle_{2} \times\langle M, M\rangle_{1} M=M \times M \times M \rightarrow M \times M$ dada por $\mu_{M}=\left(\langle M, M, M\rangle_{1},\langle M, M, M\rangle_{3}\right), \varepsilon_{M}=\left(i d_{M}, i d_{M}\right)$ e $\iota_{M}=\left(\langle M, M\rangle_{2},\langle M, M\rangle_{1}\right) . \diamond$

Exemplo 2.1.2. Dada uma variedade suave $M$, podemos construir o grupoide de referenciais de $M$, denotado por $\left(G L(T M), M, \sigma_{T M}, \tau_{T M}, \mu_{T M}, \varepsilon_{T M}, \iota_{T M}\right)$ da seguinte maneira. Os objetos são pontos de $M$. Os morfismos são isomorfismos lineares $\varphi \in \operatorname{Isovec}_{\mathbf{V}}\left(T_{x} M, T_{y} M\right)$, para cada par $x, y \in M$ (o que justifica a notação $G L(T M)$ ). As aplicações $\sigma_{T M}$ e $\tau_{T M}$ são as que fornecem os 
pontos base de domínio e codomínio dos morfismos, respectivamente. A composta $\mu_{T M}$ é a composta usual de aplicações lineares. $\varepsilon_{T M}$ é dada por $\varepsilon_{T M}(x)=i d_{T_{x} M}$, para todo $x \in M$. Finalmente, $\iota_{T M}$ é a inversão de isomorfismos lineares. Não é difícil ver que se trata realmente de um grupoide de Lie. $G L(T M)$ será transitivo, se todas as componentes conexas de $M$ tiverem mesma dimensão. $\diamond$

Existe uma relação estreita entre grupoides de Lie transitivos e fibrados principais. Grosseiramente falando, um fibrado principal consiste de uma variedade diferenciável $P$, sobre a qual age, à direita, um grupo de Lie $G_{0}$, de maneira livre e própria. Assim, um fibrado principal pode ser visto como um fibrado $\rho: P \rightarrow M$, onde $\rho$ é submersão sobrejetora e $M$ é espaço o de órbitas da ação de $G_{0}$ que, pelas condições impostas, é variedade diferenciável. Também, suas cartas fibradas são equivariantes. Trataremos de fibrados principais (e associados) de maneira um pouco mais sistemática na seção 2.3. O leitor que desejar um tratamento mais amplo poderá encontrar mais sobre fibrados principais em $[21 ; 25]$.

Fixemos um grupoide de Lie $(G, M, \sigma, \tau, \mu, \varepsilon, \iota)$. Suponha que $G$ seja transitivo. Então, todos os seus grupos de vértices são grupos de Lie isomorfos a um grupo de Lie, digamos, $G_{0}$. Pela observação 1.2.17, para cada $x \in M$ fixado, $G_{x}^{x}=\operatorname{Hom}_{G}(x, x)$ age à direita na variedade suave $P_{x}=\sigma^{-1}(x)$. A hipótese de transitividade fornece $M$ como espaço de órbitas e então, $\left(P_{x}, M, \tau_{x}, G_{x}^{x}\right)$ como fibrado principal, onde $\tau_{x}$ é $\tau$, restrita a $P_{x}$. Detalhes desta construção podem ser obtidos (com algumas adaptações) em [29].

Por outro lado, todo fibrado principal tem a ele associado um grupoide de Lie transitivo, cuja construção será dada na proposição a seguir.

Proposição 2.1.2. Seja $\left(P, \rho, M, G_{0}\right)$ um fibrado principal. Então, $P \times_{G_{0}} P$, o espaço de órbitas da ação diagonal de $G_{0}$ em $P \times P$, é espaço total de um grupoide de Lie sobre $M$.

Demonstração. Faremos apenas um esboço da demonstração. Em primeiro lugar, $P \times_{G_{0}} P$ é definido como o conjunto das classes de equivalência de pares $\left(p^{\prime}, p\right) \in P \times P$, dadas por $\left(q^{\prime}, q\right) \sim\left(p^{\prime}, p\right)$ se, e somente se, existir $g \in G_{0}$, tal que $\left(q^{\prime}, q\right)=\left(r\left(p^{\prime}, g\right), r(p, g)\right)$, onde $r: P \times G_{0} \rightarrow P$ é a ação à direita do fibrado principal. Como $G_{0}$ age livre e propriamente em $P$, assim o faz em $P \times P$, donde $P \times_{G_{0}} P$ é variedade diferenciável. Denotemos a aplicação quociente por $\rho_{P}: P \times P \rightarrow P \times_{G_{0}} P$. Definamos as aplicações estruturais do grupoide por

$$
\begin{aligned}
& \sigma\left[p^{\prime}, p\right]=\sigma \rho_{P}\left(p^{\prime}, p\right)=\rho(p) \\
& \tau\left[p^{\prime}, p\right]=\tau \rho_{P}\left(p^{\prime}, p\right)=\rho\left(p^{\prime}\right) \\
& \mu\left(\left[p^{\prime \prime}, p^{\prime}\right],\left[p^{\prime}, p\right]\right)=\left[p^{\prime \prime}, p\right] \\
& \varepsilon(\rho(p))=[p, p] \\
& \iota\left(\left[p^{\prime}, p\right]\right)=\left[p, p^{\prime}\right]
\end{aligned}
$$

para todos $p, p^{\prime}, p^{\prime \prime} \in P$. Não é difícil ver que as aplicações estão bem definidas, são suaves e satisfazem as condições de grupoide.

Definição 2.1.2. O grupoide de Lie da proposição anterior é dito grupoide de calibre do fibrado principal $P$.

Observação 2.1.1. Seja $\left(P, \rho, M, G_{0}\right)$ um fibrado principal. Considere uma variedade diferenciável $Q$, sobre a qual está definida uma ação à esquerda de $G_{0}$, digamos, $l: G_{0} \times Q \rightarrow Q$. Podemos então definir o fibrado associado $P \times_{G_{0}} Q$, como o quociente de $P \times Q$ pela relação $\left(p^{\prime}, q^{\prime}\right) \sim(p, q)$ se, e somente se, existir $g \in G_{0}$, tal que $\left(p^{\prime}, q^{\prime}\right)=\left(r(p, g), l\left(g^{-1}, q\right)\right)$. A estrutura de fibrado sobre $M$ é então dada pela projeção em $P$, seguida pela projeção $\rho$. O leitor pode encontrar detalhes sobre fibrados associados em [21] e em [25]. Vamos construir $P \times_{G_{0}} P$ como fibrado associado a $P$. Para tanto, defina a ação à esquerda $l: G_{0} \times P \rightarrow P$, por

$$
l(g, p)=r\left(p, g^{-1}\right)
$$


para todos $g \in G_{0}$ e $p \in P$. Assim, o fibrado associado construído é o fibrado alvo, $\left(P \times_{G_{0}} P, \tau, M\right)$, do grupoide de calibre de $P$. Denotando a aplicação quociente por $\rho_{P}: P \times P \rightarrow P \times_{G_{0}} P$, temos que $\left(\rho_{P}, \rho\right)$ é funtor do grupoide de pares $P \times P$, sobre $P$, no grupoide de calibre de $P$, sobre $P / G_{0}=M$. Por este motivo, o grupoide de calibre é, às vezes, denotado por $P P^{-1}$. $\diamond$

Em [29], é mostrado que estas construções que relacionam fibrados principais e grupoides de Lie transitivos são mutuamente inversas (porém, não de maneira canônica). Também não é difícil mostrar que, nestes casos, a âncora do grupoide $\alpha=(\tau, \sigma)_{\langle M, M\rangle}$ é submersão sobrejetora. Isto nos permite construir produtos fibrados de grupoides que envolvam ao menos um grupoide de Lie transitivo, pois esta é uma condição de transversalidade que garante a existência do pull-back necessário à construção apresentada na definição 1.2.7. Na mesma referência, é estabelecida uma relação entre fibrados associados a um fibrado principal e ações do grupoide de calibre deste fibrado principal ao longo de uma aplicação suave. São estas relações que nos permitem reescrever uma teoria que verse sobre objetos definidos com o auxílio de fibrados principais e seus associados, na linguagem de grupoides de Lie.

As características da categoria Man nos permitem descrições (ou versões) locais de vários objetos discutidos. De especial importância nos será o conjunto das bisseções locais de um grupoide de Lie. Evidentemente, todas as construções que não dependerem da inversão do grupoide podem ser realizadas em categorias internas a Man.

Definição 2.1.3. Seja $(G, M, \sigma, \tau, \mu, \varepsilon, \iota)$ um grupoide de Lie. Uma $\sigma$-bisseção local (ou simplesmente bisseção local) de $G$ é uma seção $\varphi: U \rightarrow G$ de $\sigma$, ou seja, $\sigma \varphi=i d_{U}$, com $U$ aberto em $M$, tal que $\tau \varphi: U \rightarrow V$ seja difeomorfismo local de $M$. O conjunto de todas as bisseções locais de $G$ é denotado por $\operatorname{Bis}_{l o c}(G)$.

Observação 2.1.2. Podemos ainda definir $\tau$-bisseções locais apenas trocando os papeis de $\sigma$ e $\tau$ na definição anterior. Estas construções são equivalentes (mesmo no caso de categorias). Também, é possível definirmos $(\tau, \sigma)$-bisseções como aplicações suaves de um aberto da base no espaço total, tais que forneçam difeomorfismos locais da base, quando compostas tanto com $\sigma$, quanto com $\tau$. A menos de menção explícita, bisseção, aqui, tem o significado de $\sigma$-bisseção. $\diamond$

Observação 2.1.3. Em geral, $\operatorname{Bis}_{l o c}(G)$ de um grupoide de Lie $(G, M, \sigma, \tau, \mu, \varepsilon, \iota)$ não tem estrutura algébrica global ${ }^{1}$. Porém, a composição de cada bisseção local com $\tau$ fornece um difeomorfismo local de $M$. Como $\varepsilon$ é bisseção local ( $M$ é aberto em $M$ ), esta construção define um subgrupoide amplo de $\operatorname{Aut}_{l o c}^{\infty}(M)$, que satisfaz a condição de feixe, ou seja, constitui um pseudogrupo de transformações de $M$ (confira o exemplo 1.2.2). $\diamond$

Observação 2.1.4. Apesar de, no caso geral, $\operatorname{Bis}_{l o c}(G)$ não possuir muita estrutura algébrica, a passagem a germes de bisseções locais fornece uma estrutura de grupoide sobre $M$. Seja $\mathcal{G}$ o conjunto de germes de bisseções de $G$. Definamos $\sigma_{\mathcal{G}}, \tau_{\mathcal{G}}: \mathcal{G} \rightarrow M$, por, para $[\varphi]_{x}$, germe de $\varphi \in \operatorname{Bis}_{\text {loc }}(G)$ em $x \in M$,

$$
\begin{gathered}
\sigma_{\mathcal{G}}[\varphi]_{x}=\sigma(\varphi(x))=x \\
\tau_{\mathcal{G}}[\varphi]_{x}=\tau(\varphi(x))
\end{gathered}
$$

Definamos a composição do grupoide. Tomemos o germe de bisseção $[\varphi]_{x}$ e chamemos $y=\tau(\varphi(x))$. Considere um germe de bisseção $[\psi]_{y}$. Tomemos $\varphi \in[\varphi]_{x}$, digamos, $\varphi: U_{x} \rightarrow G$, com $\tau \varphi: U_{x} \rightarrow U_{y}$ e $\psi \in[\psi]_{y}$, digamos, $\psi: V_{y} \rightarrow G$. Como $W_{y}=U_{y} \cap V_{y} \neq \varnothing$, pois $y \in W_{y}$, existe $\bar{\psi} \in[\psi]_{y}$, com $\operatorname{dom}(\bar{\psi})=W_{y}$. Também, existe $\bar{\varphi} \in[\varphi]_{x}, \operatorname{com} \operatorname{dom}(\bar{\varphi})=W_{x}$, onde $W_{x}=(\tau \varphi)^{-1}\left(W_{y}\right)$. Podemos então definir

$$
\mu_{\mathcal{G}}\left([\psi]_{y},[\varphi]_{x}\right)=[\mu(\bar{\psi} \tau \bar{\varphi}, \bar{\varphi})]_{x}
$$

onde os colchetes indicam que tomamos o germe da aplicação $\mu(\bar{\psi} \tau \bar{\varphi}, \bar{\varphi}): W_{x} \rightarrow G$, em $x$ (compare com o produto definido na proposição 1.2.4). É fácil ver que a aplicação $\mu(\bar{\psi} \tau \bar{\varphi}, \bar{\varphi})$ é bisseção local. Defina, para cada $x \in M$,

$$
\varepsilon_{\mathcal{G}}(x)=[\varepsilon]_{x}
$$

\footnotetext{
${ }^{1}$ Ao menos, não como estrutura sobre $M$. Entretanto, possui estrutura de grupoide sobre os abertos de $M$.
} 
Até aqui, temos uma estrutura de categoria. A verificação de que as aplicações definidas satisfazem as propriedades estruturais de categoria são cálculos diretos, envolvendo a técnica utilizada para mostrar que $\mu_{\mathcal{G}}$ está bem definida, e será deixada para o leitor. Para completar a estrutura de grupoide, definamos

$$
\iota_{\mathcal{G}}[\varphi]_{x}=\left[\iota \varphi(\tau \varphi)^{-1}\right]_{y}
$$

onde o germe é tomado em $y=\tau(\varphi(x))$. Que está bem definida, é um cálculo direto (compare novamente com a proposição 1.2.4). Para mostrar que satisfaz as propriedades de inversão em grupoide, a técnica é a mesma do que para as demais aplicações e também será deixada para o leitor. $\diamond$

Baseado na observação anterior e no fato de que jatos de aplicações podem ser vistos como homomorfismos de álgebras dadas pelo quociente de germes de funções por ideais adequados ${ }^{2}$, somos levados à seguinte definição.

Definição 2.1.4. Seja $(G, M, \sigma, \tau, \mu, \varepsilon, \iota)$ um grupoide de Lie. O r-ésimo prolongamento de $G$, ou ainda, o jato de ordem $r$ de $G$, é o grupoide (de Lie, sobre M) obtido pela projeção a jatos de ordem $r$ de todas as estruturas e objetos dados na observação 2.1.4. É denotado por $\left(J_{b}^{r} G, M, \sigma_{J^{r}}, \tau_{J^{r}}, \mu_{J^{r}}, \varepsilon_{J^{r}}, \iota_{J^{r}}\right)$, onde o sub-índice $b$ serve para distinguir entre o espaço dos $r$-jatos de bisseções de $G$ e o espaço dos $r$-jatos de seções de $G, J^{r} G$.

Ao contrário do grupoide de germes de bisseções que, em geral, tem estrutura diferenciável fraca $^{3}$, os espaços totais dos grupoides da definição anterior e as aplicações estruturais têm estrutura suave sendo, pois, grupoides de Lie. Um tratamento um pouco diferente, mas que leva aos mesmos objetos, pode ser encontrado em [28].

No caso de jatos de primeira ordem, há uma descrição direta e bastante conveniente para nossos propósitos. ${ }^{4}$ Comecemos com a descrição de jatos (de primeira ordem) de seções de fibrados. O leitor poderá encontrar detalhes em [25].

Definição 2.1.5. Seja $(E, M, \pi)$ um fibrado (geral) sobre $M$. O fibrado dos jatos de primeira ordem de $\pi$, ou ainda, prolongamento de primeira ordem de seções de $\pi$, é o fibrado afim $J E$ sobre $E$, obtido pela união disjunta das seguintes fibras: para cada e $\in E$,

$$
J_{e} E=\left\{v_{e} \in \operatorname{Hom}_{\mathbf{V e c}}\left(T_{\pi(e)} M, T_{e} E\right) \mid T_{e} \pi \circ v_{e}=i d_{T_{\pi(e)} M}\right\}
$$

onde $\operatorname{Hom}_{\mathbf{V e c}}(A, B)$ denota o espaço das aplicações lineares de $A$ em B. Tal fibrado é denotado por $\left(J E, E, \pi_{0, E}^{1}\right)$ (as cartas fibradas são construídas da maneira usual). Denotemos $\pi_{-1, E}^{0}=\pi$. Por composição, temos o fibrado sobre $M$, denotado por $\left(J E, M, \pi_{-1, E}^{1}\right)$, com $\pi_{-1, E}^{1}=\pi_{-1, E}^{0} \circ \pi_{0, E}^{1}$. $O$ fibrado dos jatos (de primeira ordem) linearizados de $\pi$ é o fibrado vetorial $\overrightarrow{J E}$ sobre $E$, obtido pela união disjunta das fibras

$$
\overrightarrow{J_{e}} E=\left\{v_{e} \in \operatorname{Hom}_{\mathbf{V e c}}\left(T_{\pi(e)} M, T_{e} E\right) \mid T_{e} \pi \circ v_{e}=0\right\}
$$

Note que $\left(J E, M, \pi_{-1, E}^{1}\right)$ é fibrado geral sobre $M$. Porém, $\left(J E, E, \pi_{0, E}^{1}\right)$ é fibrado afim sobre $E$, modelado sobre o fibrado vetorial $\vec{J} E$.

Existe uma relação íntima entre conexões gerais em fibrados e jatos de primeira ordem. Conexões podem ser definidas como seções de $\left(J E, E, \pi_{0, E}^{1}\right)$. Agora, note que, para um grupoide de Lie $(G, M, \sigma, \tau, \mu, \varepsilon, \iota)$, temos que $(G, M, \sigma)$ é fibrado geral (o mesmo vale para $\tau$ ). Usaremos este fato para descrever conexões em grupoides. Ocorre que o fibrado dos jatos de seções de $\sigma$ possui estrutura de categoria em Man, sobre $M$.

\footnotetext{
${ }^{2} \mathrm{O}$ leitor poderá encontrar um bom tratamento destas noções em [25].

${ }^{3}$ Digno de nota é que, com a topologia de feixes, este grupoide é um grupoide de Lie, a menos da condição do espaço total ser de Hausdorff [29].

${ }^{4}$ Uma descrição semelhante pode ser obtida para jatos de ordem superior, porém os cálculos são consideravelmente mais extensos. O leitor interessado poderá fazê-lo utilizando a descrição pontual de jatos de ordem superior, dada em [25].
} 
Proposição 2.1.3. Seja $(G, M, \sigma, \tau, \mu, \varepsilon, \iota)$ um grupoide de Lie. Então JG é espaço de morfismos de uma categoria em Man.

Demonstração. Definamos as aplicações fonte e alvo, respectivamente, por

$$
\begin{gathered}
\sigma_{J G}=\sigma \pi_{0, G}^{1} \\
\tau_{J G}=\tau \pi_{0, G}^{1}
\end{gathered}
$$

Como ambas são compostas de submersões sobrejetoras, existe o pull-back $J G_{\sigma_{J G}} \times_{\tau_{J G}} J G$. Definamos $\mu_{J G}: J G_{\sigma_{J G}} \times_{\tau_{J G}} J G \rightarrow J G$ como a aplicação dada por

$$
\mu_{J G}\left(v_{h}, u_{g}\right)=T_{(h, g)} \mu \circ\left(v_{h} \circ T_{g} \tau \circ u_{g}, u_{g}\right)
$$

para todo par componível $\left(v_{h}, u_{g}\right)$. Note que isto ocorre exatamente quando $(h, g)$ for par componível em $G$. Mostrar que $\mu_{J G}\left(v_{h}, u_{g}\right) \in J_{\mu(h, g)} G$ é um cálculo direto. Definamos agora $\varepsilon_{J G}: M \rightarrow J G$, por

$$
\varepsilon_{J G}(x)=T_{x} \varepsilon
$$

para todo $x \in M$. Como $\mu_{J G}$ e $\varepsilon_{J G}$ são aplicações que dependem apenas de derivadas até a primeira ordem de aplicações suaves são, elas próprias, suaves. As propriedades estruturais de categoria são provadas ao notarmos que estas aplicações são definidas como os termos de ordem 1 das aplicações definidas na observação 2.1.4. Assim, $\left(J G, M, \sigma_{J G}, \tau_{J G}, \mu_{J G}, \varepsilon_{J G}\right)$ é categoria em Man.

Passemos agora à descrição alternativa, mencionada anteriormente, de $J_{b} G=J_{b}^{1} G$.

Proposição 2.1.4. Utilizando as notações anteriores, $J_{b} G$ é subgrupoide máximo de JG.

Demonstração. Em primeiro lugar, $J_{b} G$ é o espaço de jatos de todas as seções de $\sigma$, que sejam bisseções. Para $g \in G$, isto significa que

$$
J_{b g} G=\left\{u_{g} \in J_{g} G \mid T_{g} \tau \circ u_{g} \in \operatorname{Iso}_{\mathbf{V e c}}\left(T_{\sigma(g)} M, T_{\tau(g)} M\right)\right\}
$$

Como a condição de invertibilidade é aberta (e respeita fibras), $J_{b} G$ é subvariedade aberta de $J G$ (de fato, um subfibrado, segundo $\sigma_{J G}$ ). Além disso, é a maior subvariedade de $J G$ que contenha apenas jatos de bisseções. Restrita a $J_{b} G, \mu_{J G}$ é fechada (do ponto de vista algébrico) pois, dados $v_{h}, u_{g} \in J_{b} G$, componíveis, temos

$$
\begin{aligned}
& T_{\mu(h, g)} \tau \circ \mu_{J G}\left(v_{h}, u_{g}\right)=T_{\mu(h, g)} \tau \circ T_{(h, g)} \mu \circ\left(v_{h} \circ T_{g} \tau \circ u_{g}, u_{g}\right)= \\
& =T_{(h, g)}(\tau \circ \mu) \circ\left(v_{h} \circ T_{g} \tau \circ u_{g}, u_{g}\right)=T_{h} \tau \circ v_{h} \circ T_{g} \tau \circ u_{g}
\end{aligned}
$$

e o resultado seguirá ao notarmos que as hipóteses levam a $T_{h} \tau \circ v_{h} \circ T_{g} \tau \circ u_{g} \in \operatorname{Isovec}_{\left(V^{\prime}\right.}\left(T_{\sigma)} M, T_{\tau(h)} M\right)$. Como $\varepsilon$ é bisseção de $G$, a aplicação identidade não sofre restrições (ou seja, $J_{b} G$ é subgrupoide amplo da categoria $J G$ ). Resta então definirmos a inversão $\iota_{J G}: J_{b} G \rightarrow J_{b} G$. Esta, é dada por

$$
\iota_{J G}\left(u_{g}\right)=T_{g} \iota \circ u_{g} \circ\left(T_{g} \tau \circ u_{g}\right)^{-1}
$$

para todo $u_{g} \in J_{b} G$. A demonstração das propriedades estruturais de grupoide pode ser obtida tendo-se em mente os comentários sobre os casos anteriores. O fato de $\iota_{J G}$ ser sua própria inversa, junto ao fato de $J_{b} G$ ser a maior subvariedade de $J G$ que contenha elementos inversíveis, fornecem a maximalidade de $J_{b} G$, como subgrupoide.

Observação 2.1.5. Vale a pena ressaltar que $J_{b} G$ não é, em geral, fibrado afim sobre $G$. Entretanto, $\pi_{0, G}^{1}: J_{b} G \rightarrow G$ é morfismo de grupoides de Lie. Também, se $G$ for transitivo, $J_{b} G$ também o será. $\diamond$ 
Exemplo 2.1.3. Calculemos o grupoide de jatos de bisseções locais do grupoide de pares de uma variedade suave $M$. Uma bisseção local de tal grupoide é uma aplicação suave $b: U \rightarrow V \times U$, onde $U$ e $V$ são abertos em $M$, de modo que $\langle M, M\rangle_{2} b=i d_{U}$ e $\langle M, M\rangle_{1} b: U \rightarrow V$ seja difeomorfismo. Assim, podemos escrever $b=\left(\phi, i d_{U}\right), \phi=\langle M, M\rangle_{1} b$. Para $x \in M$, temos

$$
j_{x} b=\left(T_{x} \phi, i d_{T_{x} M}\right)
$$

Como $\phi$ é difeomorfismo local em $M, T_{x} \phi$ é isomorfismo linear de $T_{x} M$ em $T_{\phi(x)} M$. Ainda, $\sigma_{J}\left(j_{x} b\right)=$ $x$, que é o ponto sobre o qual está definido o domínio de $T_{x} \phi$, e $\tau_{J}\left(j_{x} b\right)=\phi(x)$, que é o ponto sobre o qual está definido o codomínio de $T_{x} \phi$. Se $c=\left(\psi, i d_{V}\right)$ for outra bisseção local, componível com $b$, um cálculo direto mostra que

$$
\mu_{J}\left(j_{y} c, j_{x} b\right)=\left(T_{y} \psi \circ T_{x} \phi, i d_{T_{x} M}\right)
$$

Também, são válidas

$$
\begin{gathered}
\varepsilon_{J}(x)=\left(i d_{T_{x} M}, i d_{T_{x} M}\right) \\
\iota_{J}\left(j_{x} b\right)=\left(T_{\phi(x)} \phi^{-1}, i d_{T_{\phi(x)} M}\right)
\end{gathered}
$$

Em outras palavras, todo jato de bisseção de $M \times M$ define, canonicamente, um único elemento em $G L(T M)$ e tal associação preserva as estruturas de grupoide. Do fato de um isomorfismo linear entre espaços tangentes de $M$ definir um difeomorfismo local, cujo jato de primeira ordem depende apenas de derivadas primeiras (sendo portanto, único), segue que $J_{b}(M \times M) \approx G L(T M)$. $\diamond$

Existem várias relações entre o grupoide de jatos de um grupoide de Lie e o grupoide de referenciais da base. ${ }^{5}$ Uma que nos será útil é dada pela seguinte proposição.

Proposição 2.1.5. Seja $(G, M, \sigma, \tau, \mu, \varepsilon, \iota)$ um grupoide de Lie, tal que, ou seja transitivo, ou seja sobre uma variedade $M$ de dimensão $m$. Então, existe um morfismo de grupoides de Lie $\lambda: J_{b} G \rightarrow G_{\alpha_{G}} \times_{\alpha_{T M}} G L(T M)$, onde $\alpha_{T M}$ denota a âncora do grupoide de referenciais, $G L(T M)$, de $M$.

Demonstração. Como ou $G$ é transitivo, ou a variedade base tem dimensão fixa $m$, do exemplo 2.1.2, sabemos que ou $G$, ou $G L(T M)$ é grupoide de Lie transitivo. Disto, ao menos uma das âncoras $\alpha_{G}$ ou $\alpha_{T M}=\left(\tau_{T M}, \sigma_{T M}\right)$ é submersão sobrejetora, donde podemos aplicar a construção do produto fibrado de $G$ e $G L(T M)$ (definição 1.2.7). Para facilitar a notação, denotemos este produto por

$\left(G T M, M, \sigma_{G T M}, \tau_{G T M}, \mu_{G T M}, \varepsilon_{G T M}, \iota_{G T M}\right)$. Definamos então $\lambda: J_{b} G \rightarrow G T M$, por

$$
\lambda\left(u_{g}\right)=\left(g, T_{g} \tau \circ u_{g}\right)
$$

para todo $u_{g} \in J_{b} G$. Temos que $\lambda$ está bem definida, pois $T_{g} \tau \circ u_{g}$ é um isomorfismo linear de $T_{\sigma(g)} M$ em $T_{\tau(g)} M$. Além disso, para todo $u_{g} \in J_{b} G$, temos

$$
\begin{gathered}
\sigma_{G T M}\left(\lambda\left(u_{g}\right)\right)=\sigma(g)=\sigma_{J G}\left(u_{g}\right) \\
\tau_{G T M}\left(\lambda\left(u_{g}\right)\right)=\tau(g)=\tau_{J G}\left(u_{g}\right)
\end{gathered}
$$

Se $v_{h}$ e $u_{g}$ forem componíveis em $J_{b} G$, temos

$$
\begin{aligned}
& \mu_{G T M}\left(\lambda\left(v_{h}\right), \lambda\left(u_{g}\right)\right)=\mu_{G T M}\left(\left(h, T_{h} \tau \circ v_{h}\right),\left(g, T_{g} \tau \circ u_{g}\right)\right)= \\
& =\left(\mu(h, g), T_{h} \tau \circ v_{h} \circ T_{g} \tau \circ u_{g}\right)=\left(\mu(h, g), T_{\mu(h, g)} \tau \circ \mu_{J G}\left(v_{h}, u_{g}\right)\right)=\lambda\left(\mu_{J G}\left(v_{h}, u_{g}\right)\right)
\end{aligned}
$$

Finalmente, para todo $x \in M$, vale

$$
\lambda\left(\varepsilon_{J G}(x)\right)=\lambda\left(T_{x} \varepsilon\right)=\left(\varepsilon(x), i d_{T_{x} M}\right)=\varepsilon_{G T M}
$$

\footnotetext{
${ }^{5} \mathrm{O}$ leitor poderá encontrar mais sobre isto em [36].
} 
Logo, $\lambda: J_{b} G \rightarrow G_{\alpha_{G}} \times_{\alpha_{T M}} G L(T M)$ é morfismo de grupoides de Lie.

Observação 2.1.6. Sejam $(G, \sigma, \tau, \mu, \varepsilon, \iota)$ um grupoide de Lie (ou apenas uma categoria em Man), $\pi: E \rightarrow M$ uma submersão sobrejetora e $\Phi: G_{\sigma} \times_{\pi} E$ uma ação suave de $G$ sobre $\pi$. Exatamente no mesmo espírito da observação 2.1.4, podemos definir uma ação $[\Phi]: \mathcal{G} \sigma_{\mathcal{G}} \times_{\pi_{\mathcal{E}}} \mathcal{E} \rightarrow \mathcal{E}$, onde $\mathcal{G}$ denota o grupoide de germes de bisseções de $G$ e $\mathcal{E}$ denota o fibrado de germes de seções de $\pi$. A ação é definida (usando a notação da proposição referida) por, para $[\varphi]_{x} \in \mathcal{G}$, germe de bisseção em $x \in M$ e $[\psi]_{x} \in \mathcal{E}$, germe de seção em $x$,

$$
[\Phi]\left([\varphi]_{x},[\psi]_{x}\right)=\left[\Phi(\bar{\varphi}, \bar{\psi}) \circ(\tau \bar{\varphi})^{-1}\right]_{y}
$$

onde $\bar{\varphi}$ e $\bar{\psi}$ são como na observação referida e $y=\tau(\varphi(x))$. As propriedades são mostradas de maneira análoga. $\diamond$

Pelos mesmos motivos apontados em seguida à observação 2.1.4, somos levados à seguinte definição (que, evidentemente, vale também para objetos que sejam apenas categorias).

Definição 2.1.6. Sejam $(G, M, \sigma, \tau, \mu, \varepsilon, \iota)$ um grupoide de Lie, $\pi: E \rightarrow M$ uma submersão sobrejetora da variedade suave $E$ em $M$ e $\Phi: G_{\sigma} \times_{\pi} E \rightarrow E$ uma ação do grupoide de Lie $G$ sobre $\pi$. $O$ $r$-ésimo prolongamento de $\Phi$, ou ainda, o levantamento de ordem $r$ da ação $\Phi$, é a ação do grupoide de Lie $J_{b}^{r} G$ sobre a projeção $\pi_{-1, E}^{r}: J^{r} E \rightarrow M$, obtida pela projeção a jatos de ordem $r$ dos objetos e da ação dados na observação 2.1.6. Será denotada por $\Phi_{J^{r}}: J_{b}^{r} G_{\sigma_{J^{r}}} \times_{\pi_{J^{r}}} J^{r} E \rightarrow J^{r} E$.

Observação 2.1.7. Novamente, no caso de jatos de primeira ordem há uma descrição bastante conveniente desta ação. Definamos $\Phi_{J G}: J_{b} G_{\sigma_{J G}} \times_{\pi_{J G}} J E \rightarrow J E$ por, para qualquer $\left(u_{g}, v_{e}\right) \in J_{b} G_{\sigma_{J G}} \times_{\pi_{J G}} J E$,

$$
\Phi_{J G}\left(u_{g}, v_{e}\right)=T_{(g, e)} \Phi \circ\left(u_{g}, v_{e}\right) \circ\left(T_{g} \tau \circ u_{g}\right)^{-1}
$$

cujas propriedades podem ser verificadas, tendo em vista os mesmos comentários feitos para os casos anteriores. $\diamond$

É nossa intenção mostrar que, do mesmo modo que simetrias em variedades são bem descritas pela ação de um grupo, simetrias em fibrados são bem descritas pela ação de um grupoide. Por fibrado, aqui, entendemos uma submersão sobrejetora entre duas variedades suaves, tal que a imagem inversa de qualquer ponto de seu codomínio seja difeomorfa a uma mesma variedade suave modelo, de modo que o atlas do domínio consista de cartas que respeitem esta quebra local (são cartas adaptadas, que refletem a trivialidade local - ver [25]). Uma ação de um grupo em um objeto é o mesmo que definir um subgrupo dos automorfismos deste objeto. A tradução deste conceito para grupoides é um pouco mais sutil. Grupoides são estruturas que relacionam entidades através da noção de domínio e codomínio que cada elemento seu possui. Assim, a ação de grupoide em um fibrado fornece automorfismos das fibras individualmente, da fibra "fonte" para a fibra "alvo". Porém, um automorfismo (local) de um fibrado é mais do que isto. Ele define um isomorfismo entre uma vizinhança de uma fibra fonte e uma vizinhança de uma fibra alvo. Com isto em mente, vemos que os automorfismos de um fibrado não são descritos diretamente pela ação de um grupoide, mas pela ação induzida em suas bisseções. É então válido fazer a seguinte observação.

Observação 2.1.8. Sejam $(E, M, \pi)$ um fibrado suave geral, $(G, M, \sigma, \tau, \mu, \varepsilon, \iota)$ um grupoide de Lie e $\Phi: G{ }_{\sigma} \times_{\pi} E \rightarrow E$ uma ação suave de $G$ em $E$. Dada uma bisseção local $b \in \operatorname{Bis}_{l o c}(G)$, podemos definir um automorfismo local de $E$, da seguinte maneira. Façamos

$$
\begin{gathered}
\phi_{E}^{b}=\Phi\left(b \pi, i d_{E}\right) \\
\phi_{M}^{b}=\tau b
\end{gathered}
$$

Digamos que $b: U \rightarrow G$. É claro que $\phi_{E}^{b}:\left.\left.E\right|_{U} \rightarrow E\right|_{V}$ é um difeomorfismo entre $\left.E\right|_{U}$, ou seja, $E$ restrito a $\pi^{-1}(U)$, e $\left.E\right|_{V}$, que denota $E$ restrito a $\pi^{-1}(V)$, onde $V=\tau b(U)$. Basta, para tanto, 
lembrar que $I(b)$, a inversa de $b$, pode ser definida sem que seja necessária uma restrição em domínio ou codomínio e notar que $\phi_{E}^{I(b)}$ é a inversa de $\phi_{E}^{b}$. Além disso,

$$
\pi \phi_{E}^{b}=\pi \Phi\left(b \pi, i d_{E}\right)=\tau b \pi=\phi_{M}^{b} \pi
$$

ou seja, $\phi_{E}^{b}$ é um difeomorfismo fibra a fibra, que recobre $\phi_{M}^{b}$.

Por outro lado, se tomarmos o conjunto de todos os automorfismos locais de $E$, que levam vizinhanças de fibras, difeomorficamente, em vizinhanças de fibras, teremos uma entidade que possui uma noção de domínio, codomínio, produto, identidade e inversa, não sendo, entretanto, grupoide sobre $M$. É uma situação muito parecida com a do espaço $\operatorname{Bis}_{l o c}(G)$. Do mesmo modo, a passagem a germes de funções, na base, fornece uma estrutura de grupoide sobre $M$. Um elemento de tal espaço fornece um isomorfismo de uma fibra fonte a uma fibra alvo. A escolha de um subgrupoide amplo de tal grupoide fornece uma entidade que satisfaz todas as propriedades de ação de um grupoide em um fibrado. Obviamente, tal grupoide pode estar longe de ter estrutura diferenciável amena, mas isto é exatamente o análogo ao caso de variedades. A escolha de trabalharmos com grupoides de Lie, ao invés de grupoides mais gerais, como grupoides difeológicos, por exemplo, reside no fato de que todos os exemplos conhecidos das aplicações no escopo deste trabalho exibem simetrias dadas por pseudogrupos de transformações que podem ser expressos como sub-estruturas do espaço de bisseções locais de algum grupoide de Lie. A justificativa completa é uma reunião do que é exposto em $[20 ; 28 ; 36]$. $\diamond$

Com base na observação anterior, o problema de invariância ou equivariância de objetos definidos sobre um fibrado, com relação a uma ação de grupoide, traduz-se pelo seu comportamento com relação às bisseções do grupoide ou, mais precisamente, pelo seu comportamento mediante a ação induzida em bisseções. Como, localmente, analisar a ação das bisseções do grupoide original sobre o fibrado equivaleria a trocar um problema difícil por um muito mais complicado, outra estratégia deve ser adotada. O que faremos, reside no fato de que uma ação de um grupoide define um grupoide por si só, o grupoide de ação. Com isto, podemos analisar o comportamento do objeto (geralmente uma forma diferencial), nos referindo apenas a bisseções de um grupoide, e não a uma ação, parcialmente definida ${ }^{6}$. Isso posto, é essencial que analisemos a relação entre as bisseções do grupoide original e as do grupoide de ação. Comecemos por algumas definições.

Definição 2.1.7. Seja $\Phi: G_{\sigma} \times_{\pi} E \rightarrow E$ uma ação suave do grupoide de Lie $(G, M, \sigma, \tau, \mu, \varepsilon, \iota)$ no fibrado suave $(E, M, \pi)$. Dizemos que uma bisseção $B \in \operatorname{Bis}_{l o c}\left(G_{\sigma} \times_{\pi} E\right)$ é

- variável na base, ou ainda, de base variável, se existirem e, $e^{\prime} \in E$, com $\pi\left(e^{\prime}\right)=\pi(e)$, tais que $\pi \Phi B\left(e^{\prime}\right) \neq \pi \Phi B(e)$;

- vertical se, para todos $e, e^{\prime} \in E, \pi\left(e^{\prime}\right)=\pi(e) \Rightarrow \pi \Phi B\left(e^{\prime}\right)=\pi \Phi B(e)$;

- verticalmente constante se, para todos e, $e^{\prime} \in E, \pi\left(e^{\prime}\right)=\pi(e) \Rightarrow\langle\sigma, \pi\rangle_{1} B\left(e^{\prime}\right)=\langle\sigma, \pi\rangle_{1} B(e)$.

A terminologia "variável na base", indica o seguinte fato. Considere uma bisseção $B \in \operatorname{Bis}_{l o c}\left(G_{\sigma} \times_{\pi} E\right)$, variável na base. Para $e, e^{\prime} \in E$, sobre uma mesma fibra, temos $\pi \Phi B\left(e^{\prime}\right) \neq$ $\pi \Phi B(e)$. Digamos que $B(e)=(g, e)$ e $B\left(e^{\prime}\right)=\left(g^{\prime}, e^{\prime}\right)$. A condição da definição é então o mesmo que dizer que $\tau\left(g^{\prime}\right) \neq \tau(g)$, donde $g^{\prime} \neq g$. Neste caso, $\Phi B$ é um difeomorfismo local, mas não preserva a estrutura de fibrado de $E$. Este fenômeno é possível devido ao fato de $B$ representar, pontualmente, a escolha de um automorfismo local de $E$. O que ocorre é que, ao longo de uma mesma fibra, são escolhidos automorfismos diferentes, que recobrem difeomorfismos diferentes na base. Assim, esta escolha fornece uma família de difeomorfismos na base, quando se percorre a fibra. Daí o nome "variável na base".

Proposição 2.1.6. Nas hipóteses da definição anterior, uma bisseção vertical $B \in \operatorname{Bis}_{l o c}\left(G_{\sigma} \times_{\pi} E\right)$ fornece um difeomorfismo local de $E$, que preserva $\pi$.

\footnotetext{
${ }^{6} \mathrm{O}$ problema da invariância sob a ação de um grupoide reside justamente no fato de que a ação é parcialmente definida, o que dificulta o uso de ferramentas globais - como o pull-back da ação, por exemplo.
} 
Demonstração. Claramente, o difeomorfismo local em si, é dado por $\Phi B$. A condição de que, para todos $e, e^{\prime} \in E, \pi\left(e^{\prime}\right)=\pi(e)$ implica em $\pi \Phi B\left(e^{\prime}\right)=\pi \Phi B(e)$, permite definir $\phi_{M}$, com domínio na projeção do domínio de $B$ e codomínio na projeção do codomínio de $\Phi B$, por

$$
\phi_{M} \pi=\pi \Phi B
$$

que é claramente um difeomorfismo local em $M$, recoberto por $\Phi B$.

Proposição 2.1.7. Nas hipóteses da definição anterior, uma bisseção $B \in \operatorname{Bis}_{l o c}\left(G_{\sigma} \times_{\pi} E\right)$ é verticalmente constante se, e somente se, for induzida por uma bisseção $b \in \operatorname{Bis}_{l o c}(G)$.

Demonstração. Dada $b \in \operatorname{Bis}_{l o c}(G)$, definamos

$$
B=\left(b \pi, i d_{E}\right)_{\langle\sigma, \pi\rangle}
$$

cujo domínio é a imagem inversa, por $\pi$, do domínio de $b$. Agora,

$$
\langle\sigma, \pi\rangle_{2} B=\langle\sigma, \pi\rangle_{2}\left(b \pi, i d_{E}\right)_{\langle\sigma, \pi\rangle}=i d_{E_{U}}
$$

mostrando que $B$ é seção de $\langle\sigma, \pi\rangle_{2}$, onde $i d_{E_{U}}$ denota a identidade no domínio de $B$. Para que $B$ seja bisseção, resta mostrar que $\Phi B$ tem inversa. Definamos $\tilde{B}=\left(I(b) \pi, i d_{E}\right)$. Um cálculo análogo ao feito na proposição 1.2.5, mostra que $\Phi \tilde{B}$ é inversa de $\Phi B$. Como $B$ é definida como composta de aplicações suaves, a diferenciabilidade não é um problema. Finalmente, se $e, e^{\prime} \in \operatorname{dom}(B)$ estiverem sobre uma mesma fibra de $\pi$, temos

$$
\langle\sigma, \pi\rangle_{1} B\left(e^{\prime}\right)=b \pi\left(e^{\prime}\right)=b \pi(e)=\langle\sigma, \pi\rangle_{1} B(e)
$$

donde é verticalmente constante.

Por outro lado, tomemos $B \in \operatorname{Bis}_{l o c}\left(G_{\sigma} \times_{\pi} E\right)$, verticalmente constante. A condição de verticalidade constante permite que definamos $b: \pi(\operatorname{dom}(B)) \rightarrow G$, suave, por

$$
b \pi=\langle\sigma, \pi\rangle_{1} B
$$

Então,

$$
\sigma b \pi=\sigma\langle\sigma, \pi\rangle_{1} B=\pi
$$

Como $\pi$ é submersão sobrejetora, temos

$$
\sigma b=i d_{U}
$$

onde $U=\pi(\operatorname{dom}(B))$. Para que $b$ seja bisseção, resta mostrar que $\tau b$ é difeomorfismo local em $M$. Calculemos, para tanto,

$$
\tau b \pi=\tau\langle\sigma, \pi\rangle_{1} B=\pi \Phi B
$$

que mostra que $\tau b$ é o difeomorfismo local em $M$, recoberto por $\Phi B$, elaborado na proposição anterior. Logo, $b \in \operatorname{Bis}_{l o c}(G)$.

Nas aplicações em que estamos interessados, as fibras de um fibrado desempenham um papel de "espaço interno", ou seja, graus de liberdade adicionais aos do espaço de interesse, que é a base do fibrado. Portanto, ao considerarmos uma simetria do fibrado, ou seja, uma escolha de automorfismos, tal escolha deve refletir este conceito. Em outras palavras, em uma mesma fibra opera um mesmo automorfismo. Torna-se evidente então que, para fins de invariância (ou equivariância), de todas as bisseções de $\operatorname{Bis}_{l o c}\left(G_{\sigma} \times_{\pi} E\right)$, nos interessam aquelas que forem verticalmente constantes. Este comentário é especialmente válido nos casos em que as fibras têm alguma estrutura que deva ser respeitada por um automorfismo, como no caso de fibrados principais, por exemplo. Porém, vale ressaltar que bisseções que não sejam verticalmente constantes podem ser úteis para modelar 
casos em que a teoria verse sobre seções de um fibrado, por exemplo, e a simetria ao qual o sistema está submetido dependa da seção. Não faremos isso aqui.

Resta ainda relacionar as bisseções de um grupoide de ação que nos serão úteis e seus prolongamentos.

Proposição 2.1.8. Dada uma ação $\Phi: G_{\sigma} \times_{\pi} E \rightarrow E$, como acima, o subconjunto de $\operatorname{Bis}\left(J_{b}^{r} G_{\sigma_{J^{r}}} \times_{\pi_{J^{r}}} J^{r} E\right)$ constituído por prolongamentos de ordem $r$ de bisseções verticalmente constantes de $\operatorname{Bis}\left(G_{\sigma} \times_{\pi} E\right)$, é subgrupo do grupo das bisseções verticalmente constantes de $\operatorname{Bis}\left(J_{b}^{r} G_{\sigma_{J^{r}}} \times_{\pi_{J^{r}}} J^{r} E\right)$.

Demonstração. Pela proposição anterior, uma bisseção verticalmente constante de $\operatorname{Bis}\left(G_{\sigma} \times{ }_{\pi} E\right)$ é da forma

$$
B=\left(b \pi, i d_{E}\right)_{\langle\sigma, \pi\rangle}
$$

onde $b \in \operatorname{Bis}(G)$. Dado que $B$ é morfismo estrito de fibrados sobre $M$, faz sentido falar em seu prolongamento de ordem $r$, que então tem a forma

$$
j^{r} B=\left(j^{r} b j^{r} \pi, i d_{J^{r} E}\right)=\left(j^{r} b \pi_{J^{r}}, i d_{J^{r} E}\right)
$$

Pela mesma proposição e do fato de $j^{r} b$ ser bisseção de $J_{b}^{r} G$, segue que $j^{r} B$ é verticalmente constante. Por definição, o prolongamento de ordem $r$ é homomorfismo de grupos. Como, pela proposição 1.2.5, as bisseções verticalmente constantes constituem um subgrupo do grupo de bisseções, segue o resultado.

Passemos agora ao conceito de forma diferencial multiplicativa, de importância central nesta tese. Há muito sobre formas multiplicativas na literatura. O leitor poderá encontrar uma reunião de fatos, no espírito em que serão utilizados aqui, em $[9 ; 36]$.

Definição 2.1.8. Seja $(G, M, \sigma, \tau, \mu, \varepsilon, \iota)$ um grupoide de Lie. Uma forma diferencial $\omega \in \Omega^{\bullet}(G)$ é dita multiplicativa, se satisfizer

$$
\mu^{*} \omega=\langle\sigma, \tau\rangle_{1}^{*} \omega+\langle\sigma, \tau\rangle_{2} \omega
$$

onde $\mu^{*} \omega,\langle\sigma, \tau\rangle_{1}^{*} \omega$ e $\langle\sigma, \tau\rangle_{2} \omega$ denotam os pull-backs da forma $\omega$, pelas respectivas aplicações, $\grave{a}$ variedade $G_{\sigma} \times_{\tau} G$.

Uma interpretação da condição de multiplicatividade de uma forma diferencial, em termos de cociclos em um complexo adequado, pode ser encontrada em [2].

Definição 2.1.9. Seja $\omega$ uma forma diferencial multiplicativa em um grupoide de Lie $(G, M, \sigma, \tau, \mu, \varepsilon, \iota)$. Dizemos que uma bisseção $b \in \operatorname{Bis}(G)$ é $\omega$-holônoma, quando $b^{*} \omega=0$.

Observação 2.1.9. A condição de multiplicatividade de $\omega$ garante que o conjunto das bisseções $\omega$-holônomas seja subgrupo de $\operatorname{Bis}(G)$, como observado em [36]. Se encararmos $\omega$ como um sistema de equações diferenciais, a equação $b^{*} \omega=0$ diz que o par $(M, b)$ é uma variedade integral de $\omega$. $\diamond$

Agora estamos aptos a definir o conceito de invariância de uma forma diferencial em um fibrado, pela ação de um grupoide de Lie.

Definição 2.1.10. Sejam $\Phi: G{ }_{\sigma} \times{ }_{\pi} E \rightarrow E$ uma ação suave de um grupoide de Lie $(G, M, \sigma, \tau, \mu, \varepsilon, \iota)$ em um fibrado $(E, M, \pi)$ e $\omega \in \Omega^{\bullet}(E)$ uma forma diferencial em $E$. Denote por $\omega_{\Phi}$ a forma diferencial (multiplicativa, em $G_{\sigma} \times_{\pi} E$ ) definida por

$$
\omega_{\Phi}=\langle\sigma, \pi\rangle_{2}^{*} \omega-\Phi^{*} \omega
$$

Diremos que $\omega$ é absolutamente invariante por $\Phi$ (ou pela ação de $G$, quando não houver risco de confusão), quando toda bisseção de $G_{\sigma} \times_{\pi} E$ for $\omega_{\Phi}$-holônoma. Diremos que $\omega$ é invariante por $\Phi$, quando toda bisseção verticalmente constante de $G_{\sigma} \times_{\pi} E$ for $\omega_{\Phi}$-holônoma. Mais geralmente, 
dado $\mathcal{G}$ um subgrupo de $\operatorname{Bis}\left(G_{\sigma} \times_{\pi} E\right)$, diremos que $\omega$ é $\mathcal{G}$-invariante, se todo elemento de $\mathcal{G}$ for $\omega_{\Phi}$-holônomo.

Que seja de meu conhecimento, a definição anterior não foi apresentada em nenhum outro lugar na literatura, até o presente momento.

Observação 2.1.10. É interessante notar (e é fato bem conhecido) que, dados um grupoide de Lie $(G, M, \sigma, \tau, \mu, \varepsilon, \iota)$ e uma forma diferencial $\omega \in \Omega^{\bullet}(M)$, definida em sua base, a forma diferencial

$$
\omega_{G}=\sigma^{*} \omega-\tau^{*} \omega
$$

é multiplicativa. $\diamond$

Observação 2.1.11. Considere um fibrado $(E, M, \pi)$, sobre o qual age um grupo de transformações, ou seja, é escolhido um subgrupo $\mathcal{G}$ dos automorfismos de $E$ (possivelmente de dimensão infinita). Suponha que tenhamos a sorte de tal grupo poder ser descrito como subgrupo do grupo de bisseções de algum grupoide de Lie $(G, M, \sigma, \tau, \mu, \varepsilon, \iota)$. Assim, temos definida uma ação $\Phi: G{ }_{\sigma} \times{ }_{\pi} E \rightarrow E$, que descreve a ação de $\mathcal{G}$ em $E$, associando a cada elemento $\phi \in \mathcal{G}$, uma bisseção verticalmente constante $B \in \operatorname{Bis}\left(G_{\sigma} \times_{\pi} E\right)$, com $\phi=\Phi B$. Denotemos este subgrupo de bisseções também por $\mathcal{G}$. Considere uma forma diferencial $\omega \in \Omega^{\bullet}(E), \mathcal{G}$-invariante. Isto significa que, dado $B \in \mathcal{G}$,

$$
\begin{aligned}
& B^{*} \omega_{\Phi}=0 \\
\therefore \quad & B^{*}\langle\sigma, \pi\rangle_{2}^{*} \omega-B^{*} \Phi^{*} \omega=0 \\
\therefore \quad & \left(\langle\sigma, \pi\rangle_{2} B\right)^{*} \omega=(\Phi B)^{*} \omega \\
\therefore & \omega=(\Phi B)^{*} \omega
\end{aligned}
$$

Como $\phi=\Phi B$ é um difeomorfismo que preserva fibras, elemento do grupo original considerado, a última equação reflete exatamente o conceito de forma invariante sob a ação de um grupo de automorfismos de um fibrado, presente na literatura ${ }^{7}$. A diferença é que, apesar de $\mathcal{G}$ ter dimensão infinita, a possibilidade de considerá-lo como bisseções de um grupoide de Lie fornece uma descrição que, localmente, tem dimensão finita. $\diamond$

\section{$2.2 \quad$ Algebroides de Lie}

Algebroides de Lie são objetos de natureza algébrica, cujo estudo pode ser feito de maneira totalmente independente do de grupoides de Lie. Sua essência algébrica independe até mesmo do conceito de variedade, podendo ser formulados (obviamente de maneira mais geral) em termos de álgebras e módulos ${ }^{8}$. Aqui, nos ateremos a sua apresentação em termos de fibrados, pois nosso objetivo é descrever dados infinitesimais de grupoides de Lie.

Definição 2.2.1. Um algebroide de Lie é uma quíntupla $(E, M,[],, \pi, a)$, onde

i) $(E, M, \pi)$ é um fibrado vetorial suave;

ii) $(\Gamma(E),[]$,$) é uma \mathbb{R}$-álgebra de Lie, onde $\Gamma(E)$ denota o espaço de seções suaves de $\pi$;

iii) $a: E \rightarrow T M$ é um morfismo estrito de fibrados vetoriais sobre $M$, dita a âncora do algebroide de Lie;

satisfazendo as seguintes condições:

iv) $\Gamma(a): \Gamma(E) \rightarrow \mathfrak{X}(M)$ é um homomorfismo de álgebras de Lie, onde $\mathfrak{X}(M)$ denota o espaço dos campos de vetores sobre $M$, com o colchete de Lie usual de campos;

\footnotetext{
${ }^{7}$ Veja, por exemplo, [16].

${ }^{8}$ Esta é a formulação em termos de álgebras de Lie-Rinehart, originalmente apresentada em [34].
} 
v) é válida a seguinte "regra de Leibniz": para todos $\xi, \eta \in \Gamma(E)$ e toda $f \in C^{\infty}(M)$,

$$
[\xi, f \eta]=f[\xi, \eta]+\left(\mathcal{L}_{\Gamma a(\xi)} f\right) \eta
$$

onde $\mathcal{L}_{\Gamma а(\xi)} f$ denota a derivada de Lie da função $f$, ao longo do campo vetorial $\Gamma(a)(\xi)$.

Observação 2.2.1. Algumas observações sobre a definição anterior. Em primeiro lugar, de agora em diante denotaremos $\Gamma(a)$ simplesmente por $a$, pois acredito não haver risco de confusão e tal abreviação melhora a legibilidade das equações.

Em segundo lugar, vale notar que as condições $i v$ ) e $v$ ) acima, neste caso, não são independentes. Claramente, $v$ ) é possível devido ao fato de que $E$, sendo fibrado vetorial sobre $M$, fornece $\Gamma(E)$ como $C^{\infty}(M)$-módulo projetivo e finitamente gerado. Mediante esta condição, $v$ ) implica $\left.i v\right)$. Em casos mais gerais (álgebras de Lie-Rinehart), nos quais a álgebra de Lie não seja um módulo projetivo e finitamente gerado sobre a álgebra associativa base, tais condições são independentes. Apesar de tais casos não serem considerados aqui, manter a condição $i v$ ) explícita na definição anterior me parece fornecer uma melhor intuição sobre o que devemos esperar do comportamento de tal objeto, bem como seu significado.

Por último, a condição $v$ ) pode ser reescrita como

$$
[\xi, f \eta]-f[\xi, \eta]=\left(\mathcal{L}_{a(\xi)} f\right) \eta
$$

que significa que o operador $\operatorname{Ad}(\xi)$, dado por $\operatorname{Ad}(\xi)(\eta)=[\xi, \eta]$, é um operador diferencial de primeira ordem em $\Gamma(E)$. Mais que isso, é um endomorfismo derivativo de $\Gamma(E)$ (confira $[26 ; 27]$ ). $\diamond$

A noção de morfismos de algebroides de Lie reflete o fato de seu ambiente natural ser o algébrico. Enquanto é relativamente pouco dispendioso definir morfismos de álgebras de Lie-Rinehart (o que não faremos aqui), a definição de morfismos de algebroides de Lie, em termos de fibrados, é consideravelmente mais elaborada, devido principalmente ao fato de que morfismos de fibrados, em geral, não induzem morfismos em seus espaços de seções, ingrediente essencial para este conceito. Este problema pode ser evitado se nos ativermos ao caso de morfismos de algebroides de Lie sobre uma mesma base. Para a definição geral, o leitor poderá consultar [17].

Definição 2.2.2. Sejam $\left(E, M,[,]_{E}, \pi, a\right)$ e $\left(E^{\prime}, M,[,]_{E^{\prime}}, \pi^{\prime}, a^{\prime}\right)$ dois algebroides de Lie. Um morfismo estrito de fibrados vetoriais (ou seja, que recobre a identidade em $M$ ) $\varphi: E \rightarrow E^{\prime}$ é dito um pré-morfismo de algebroides de Lie, se satisfizer

$$
a^{\prime} \varphi=a
$$

A aplicação $\varphi$ é dita morfismo de algebroides de Lie se, além disso, $\Gamma \varphi$ for homomorfismo de álgebras de Lie.

Neste ponto, o leitor deve ser alertado de que a discriminação entre morfismos e pré-morfismos, na definição anterior, não é usual na literatura. Acho interessante fazê-lo apenas porque o conceito de pré-morfismo é útil ao lidarmos com conexões. A condição do morfismo $\varphi$ induzir um homomorfismo de álgebras de Lie está relacionada à curvatura de um conceito de conexão subjacente. O leitor poderá encontrar mais detalhes em $[1 ; 17 ; 26]$.

O conteúdo da próxima proposição é padrão na literatura sobre grupoides de Lie e, portanto, será apresentado apenas um esboço de sua demonstração. O leitor poderá encontrar os detalhes, por exemplo, em [29].

Proposição 2.2.1. Seja $(G, M, \sigma, \tau, \mu, \varepsilon, \iota)$ um grupoide de Lie. Existe um algebroide de Lie associado a $G$.

Demonstração. Como $\sigma$ é submersão sobrejetora, o espaço $V^{\sigma} G=\operatorname{ker}(T \sigma)$ é subfibrado vetorial de $T G$. Como $\varepsilon$ é mergulho próprio, $V^{\sigma} G$, restrito à imagem de $\varepsilon$, é fibrado vetorial sobre $M$. 
Chamemos esta estrutura de fibrado vetorial de $\left(\mathfrak{A} G, M, \pi_{A}\right)$. Como $G$ age naturalmente em $V^{\sigma} G$ (por translações à direita), podemos falar em campos de vetores $\sigma$-verticais, invariantes à direita. O conjunto de tais campos é fechado pelo colchete de Lie em TG. Como cada campo vetorial $\sigma$ vertical e invariante à direita determina e é determinado por um único elemento de $\mathfrak{A} G$, isto induz um colchete de Lie em $\Gamma(\mathfrak{A} G)$. Para a âncora do algebroide de Lie, tomemos $T \tau$, restrita a $\mathfrak{A} G$. Não é difícil verificar (apesar de um pouco trabalhoso), que $T \tau$, restrita a $\mathfrak{A} G$, denotada por $a_{A}$, satisfaz as propriedades de âncora, sendo portanto, $\left(\mathfrak{A} G, M,[],, \pi_{A}, a_{A}\right)$, um algebroide de Lie.

Definição 2.2.3. Nas condições da proposição anterior, dizemos que $\mathfrak{A} G$ é o algebroide de Lie do grupoide de Lie G.

É natural, neste ponto, que nos perguntemos qual a relação entre jatos de bisseções de grupoides e seus algebroides de Lie. Em [28], o leitor poderá encontrar a demonstração do seguinte fato.

Proposição 2.2.2. Seja $(G, M, \sigma, \tau, \mu, \varepsilon, \iota)$ um grupoide de Lie. Então,

$$
\mathfrak{A}\left(J_{b} G\right) \approx J \mathfrak{A} G
$$

Exemplo 2.2.1. Considere o grupoide de pares $\left(M \times M, \sigma_{M}, \tau_{M}, \mu_{M}, \varepsilon_{M}, \iota_{M}\right)$ de uma variedade suave $M$. O algebroide de Lie de $M \times M$ tem como espaço total o núcleo de $T\langle M, M\rangle_{2}$, restrito à diagonal, ou seja, $\mathfrak{A}(M \times M)=\left.(T M \times\{0\})\right|_{M} \approx T M$. O colchete de Lie é, obviamente, o colchete usual de campos de vetores em $T M$. A âncora é dada pela projeção $T\langle M, M\rangle_{1}: T M \times\{0\} \rightarrow T M$, sendo portanto, equivalente à identidade em $T M$. Assim, o algebroide de Lie de $M \times M$ é o fibrado tangente $T M$. Da proposição anterior e do fato de ser $G L(T M)=J_{b}(M \times M)$, temos que

$$
\mathfrak{A}(G L(T M)) \approx J(T M)
$$

$\diamond$

Quando um grupoide de Lie age sobre um fibrado, define um grupoide de Lie sobre o espaço total do fibrado. Cabe então perguntar qual a relação do algebroide de Lie deste grupoide com o do grupoide original. As demonstrações das proposições a seguir podem ser encontradas em [17] e mais detalhes em [26].

Proposição 2.2.3. Seja $\Phi: G_{\sigma} \times_{\pi} E \rightarrow E$ uma ação suave do grupoide de Lie $(G, M, \sigma, \tau, \mu, \varepsilon, \iota)$ no fibrado $(E, M, \pi)$. Então, o espaço $\mathfrak{A} G_{\pi_{A}} \times_{\pi} E$ tem estrutura de algebroide de Lie sobre $E$, dada por

i) $a_{E}: \mathfrak{A} G_{\pi_{A}} \times_{\pi} E \rightarrow T E$ é dada por

$$
a_{E}(\xi, e)=T \Phi^{e}(\xi)
$$

para todo $(\xi, e) \in \mathfrak{A} G_{\pi_{A}} \times_{\pi} E$, onde $\Phi^{e}:\left.G\right|_{\sigma^{-1}(\pi(e))} \rightarrow E$ é dada por $\Phi^{e}(g)=\Phi(g, e) ;$

ii) o colchete em $\Gamma\left(\mathfrak{A} G_{\pi_{A}} \times_{\pi} E\right) \approx C^{\infty}(E) \otimes_{C^{\infty}(M)} \Gamma(\mathfrak{A} G)$, é dado, em elementos, por

$$
[f \otimes \xi, g \otimes \eta]=f g \otimes[\xi, \eta]+f a_{E}(\xi)(g) \otimes \eta-g a_{E}(\eta)(f) \otimes \xi
$$

para todas $f, g \in C^{\infty}(E)$ e todos $\xi, \eta \in \Gamma(\mathfrak{A} G)$, lembrando que $a_{E}$ na equação anterior é a $\Gamma\left(a_{E}\right)$ do primeiro item, donde $a_{E}(f \otimes \xi)=f a_{E}(\xi)$.

Proposição 2.2.4. Nas hipóteses da proposição anterior, o algebroide ali construído é canonicamente isomorfo ao algebroide de Lie do grupoide de ação, ou seja,

$$
\mathfrak{A}\left(G_{\sigma} \times_{\pi} E\right) \approx \mathfrak{A} G_{\pi_{A}} \times_{\pi} E
$$

como algebroides de Lie. 
Vale notar que, assim como no caso de grupos e álgebras de Lie, o conceito de ação (infinitesimal) de um algebroide de Lie em um fibrado faz sentido por si só. Porém, no presente caso, as questões de integrabilidade são muito mais delicadas. O leitor interessado poderá encontrar a definição de ação de um algebroide de Lie sobre um fibrado, bem como detalhes adicionais, em [26].

Em $[9 ; 36]$ o leitor poderá encontrar detalhes sobre a proposição a seguir, cuja importância é central neste trabalho e cujo conteúdo pode ser resumido no seguinte caso especial, que pode ser demonstrado com um cálculo direto ${ }^{9}$.

Proposição 2.2.5. Sejam $(G, M, \sigma, \tau, \mu, \varepsilon, \iota)$ um grupoide de Lie e $\theta \in \Omega^{k}(M)$ uma $k$-forma diferencial sobre $M$. Então, existem uma aplicação linear $D_{\theta}: \Gamma(\mathfrak{A} G) \rightarrow \Omega^{k}(M)$ e um morfismo estrito de fibrados vetoriais $l_{\theta}: \mathfrak{A} G \rightarrow \bigwedge^{k-1} T^{*} M$, que satisfazem

i) $D_{\theta}(f \xi)=f D_{\theta}(\xi)+d f \wedge l_{\theta}(\xi)$;

ii) $D_{\theta}([\xi, \eta])=\mathcal{L}_{a(\xi)} D_{\theta}(\eta)-\mathcal{L}_{a(\eta)} D_{\theta}(\xi)$;

iii) $l_{\theta}([\xi, \eta])=\mathcal{L}_{a(\xi)} l_{\theta}(\eta)-i_{a(\eta)} D_{\theta}(\xi)$;

iv) $i_{a(\xi)} l_{\theta}(\eta)=-i_{a(\eta)} l_{\theta}(\xi)$;

para todos $\xi, \eta \in \mathfrak{A} G$ e toda $f \in C^{\infty}(M)$, dadas por

$$
\begin{gathered}
D_{\theta}(\xi)=\mathcal{L}_{a(\xi)} \theta \\
l_{\theta}(\xi)=i_{a(\xi)} \theta
\end{gathered}
$$

para todo $\xi \in \mathfrak{A} G$.

Observação 2.2.2. Na proposição anterior, o par $\left(D_{\theta}, l_{\theta}\right)$ é dito operador de Spencer sobre $\mathfrak{A} G$. Suponha que $\omega=d \theta$. Então, são válidas

$$
\begin{gathered}
D_{\omega}(\xi)=d D_{\theta}(\xi) \\
l_{\omega}(\xi)=D_{\theta}(\xi)-d l_{\theta}(\xi)
\end{gathered}
$$

para todo $\xi \in \mathfrak{A} G$. Claramente, qualquer que seja $\theta \in \Omega^{k}(M), l_{\theta}$ induz uma aplicação $C^{\infty}(M)$ linear $\mathcal{J}: \Gamma(\mathfrak{A} G) \rightarrow \Omega^{k-1}(M)$. A súbita mudança de nome será justificada quando considerarmos correntes de Noether, no próximo capítulo. $\diamond$

É suficiente dizer que a proposição anterior fornecerá o conceito de correntes, quando o grupoide de Lie considerado for o grupoide de ação, para uma ação que forneça as simetrias do fibrado considerado. Por hora, será útil uma análise de fibrados principais e associados, do ponto de vista de grupoides e algebroides de Lie.

\subsection{Um pouco sobre fibrados principais e associados}

Nesta seção, trataremos de alguns tópicos referentes a fibrados principais e associados, usando a linguagem de grupoides de Lie aqui descrita. Longe de apresentar um tratamento completo, sequer auto-contido, esta seção tem o propósito de preparar a arena na qual se desenvolvem as teorias de calibre, objeto de destaque no próximo capítulo. Muito do que é feito aqui tem como ponto de partida o texto [25].

Fixemos algumas notações. Nesta seção, $(G, m, e, i)$ denotará um grupo de Lie. Um fibrado principal é caracterizado por uma ação à direita, suave $r: P \times G \rightarrow P$, do grupo de Lie $G$ na variedade suave $P$, que é livre, ou seja, em que o único elemento de $G$ que fixa pontos é a unidade, e própria, ou seja, a imagem inversa de qualquer subconjunto compacto em $P \times P$ pela aplicação

\footnotetext{
${ }^{9} \mathrm{~A}$ proposição, como consta nos referidos textos, é muito mais geral do que o apresentado aqui. Sua apresentação foi simplificada ao suficiente para nossos propósitos.
} 
$\left(r,\langle P, G\rangle_{1}\right)_{\langle P, P\rangle}$, é um compacto. A condição de $r$ ser própria garante que o espaço de órbitas $M=P / G$ tenha uma estrutura diferenciável e, em conjunto com a de ser livre, que seja variedade suave. A aplicação quociente $\rho: P \rightarrow M$ fornece então estrutura de fibrado, cuja fibra típica é $G$. Também, por construção, $r$ é transitiva nas fibras de $P$. Assim, $(P, M, \rho, G)$ denotará um fibrado principal, ficando implícita a ação $r$ (que, de qualquer modo, pode ser reconstruída com estes dados).

Das propriedades da ação $r$, podemos construir uma aplicação diferença, denotada por $\delta: P{ }_{\rho} \times{ }_{\rho} P \rightarrow G$ e definida, implicitamente, por

$$
r\left(p, \delta\left(p, p^{\prime}\right)\right)=p^{\prime}
$$

para todo $\left(p, p^{\prime}\right) \in P{ }_{\rho} \times{ }_{\rho} P$, ou seja, todos $p, p^{\prime} \in P$, com $\rho\left(p^{\prime}\right)=\rho(p)$. Reuniremos algumas propriedades notáveis da aplicação diferença em uma proposição, cujas demonstrações serão omitidas, por serem cálculos diretos.

Proposição 2.3.1. A aplicação diferença $\delta: P{ }_{\rho} \times{ }_{\rho} P \rightarrow G$, acima definida, satisfaz as seguintes propriedades:

i) $\delta(p, p)=e$.

ii) $\delta\left(p, p^{\prime}\right)=i\left(\delta\left(p^{\prime}, p\right)\right)$.

iii) $\delta\left(p, r\left(p^{\prime}, g\right)\right)=m\left(\delta\left(p, p^{\prime}\right), g\right)$, e portanto, $\delta(p, r(p, g))=g$.

iv) $m\left(\delta\left(p^{\prime \prime}, p^{\prime}\right), \delta\left(p^{\prime}, p\right)\right)=\delta\left(p^{\prime \prime}, p\right)$.

v) $\delta\left(r\left(p^{\prime \prime \prime}, \delta\left(p^{\prime \prime}, p^{\prime}\right)\right), r\left(p^{\prime \prime \prime}, \delta\left(p^{\prime \prime}, p\right)\right)\right)=\delta\left(p^{\prime}, p\right)$.

vi) $\delta\left(r\left(p^{\prime}, g\right), r(p, g)\right)=\operatorname{Ad}(i(g))\left(\delta\left(p^{\prime}, p\right)\right)$.

onde $p, p^{\prime}, p^{\prime \prime}, p^{\prime \prime \prime}$ são elementos de $P$ sobre uma mesma fibra, $g \in G$ e $\operatorname{Ad}(g): G \rightarrow G$ é a aplicação adjunta, dada por

$$
\operatorname{Ad}(g)(h)=m(m(g, h), i(g))
$$

Um fibrado associado a $P$ é caracterizado por uma variedade suave $Q$, sobre a qual age, à esquerda, o grupo de Lie $G$. Denotemos esta ação por $l: G \times Q \rightarrow Q$. A estrutura de fibrado é dada da seguinte maneira. Temos uma ação conjunta $(P \times Q) \times G \rightarrow P \times Q$, dada por

$$
((p, q), g) \mapsto(r(p, g), l(i(g), q))
$$

O espaço total do fibrado é a variedade quociente $P \times_{G} Q$ (cuja estrutura suave é garantida pelas propriedades de $r$ ), de modo que $\rho_{Q}: P \times Q \rightarrow P \times{ }_{G} Q$ tem estrutura de fibrado principal, sendo o fibrado associado em questão, a estrutura $\pi_{Q}: P \times_{G} Q \rightarrow M$, dada pelo diagrama

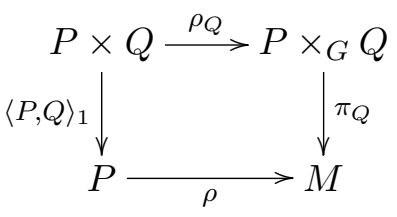

De maneira semelhante ao caso anterior (ver [25]), podemos definir a aplicação deslocamento $\delta_{Q}: P{ }_{\rho} \times_{\pi_{Q}}\left(P \times_{G} Q\right) \rightarrow Q$, dada em elementos, por

$$
\delta_{Q}\left(p, \rho_{Q}\left(p^{\prime}, q\right)\right)=l\left(\delta\left(p, p^{\prime}\right), q\right)
$$

Isto é possível graças ao fato de que, para $p$ fixo, a aplicação $\delta_{Q}^{p}:\left.\left(P \times_{G} Q\right)\right|_{\rho(p)} \rightarrow Q$, tal que $\delta_{Q}^{p}\left(\rho_{Q}\left(p^{\prime}, q\right)\right)=\delta_{Q}\left(p, \rho_{Q}\left(p^{\prime}, q\right)\right)$, é um difeomorfismo. 
Se $(E, M, \pi)$ for um fibrado geral, uma conexão em $E$ é uma seção $\nabla: E \rightarrow J E$, de $\pi_{0, E}^{1}: J E \rightarrow E$. Uma conexão principal, no fibrado principal $(P, M, \rho, G)$ é uma conexão em $P$, compatível com a ação de grupo, ou seja, uma seção $\nabla: P \rightarrow J P$, tal que

$$
\nabla(r(p, g))=r_{J P}(\nabla(p), g)
$$

onde $r_{J P}: J P \times G \rightarrow J P$ é dada por

$$
r_{J P}\left(j_{x} \varphi, g\right)=j_{x}(r(\varphi, g))
$$

para toda seção local $\varphi: U \rightarrow P$, com $x \in U$. Definamos $C P$, como o espaço de órbitas desta ação, ou seja, $C P=J P / G$. Temos então o diagrama

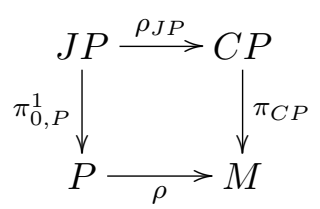

Deste modo, uma conexão principal pode ser vista como uma seção $A: M \rightarrow C P$, de $\pi_{C P}: C P \rightarrow M$. Um elemento $A(x) \in C P$ é descrito pelo jato de primeira ordem de uma seção $s: U \rightarrow P$, e de qualquer outra seção na forma $\tilde{s}=r(s, g)$, com $g \in G$. Ao fibrado $\left(C P, M, \pi_{C P}\right)$, damos o nome de fibrado de conexões de $P$. O leitor pode encontrar mais sobre isto (tais como a prova de que estes objetos são suaves) em [25].

$\mathrm{Na}$ mesma referência, o leitor poderá encontrar a seguinte noção de derivada absoluta, ou como preferimos, derivada covariante de uma seção de $P \times{ }_{G} Q$, com relação a uma conexão principal em $P$ (contudo, com uma notação um pouco diferente). Note que, devido ao diagrama 2.1, qualquer seção local $\psi: U \rightarrow P \times_{G} Q$ pode ser escrita como $\psi=\rho_{Q}(\varphi, f)$, onde $\varphi: U \rightarrow P$ é uma seção de $\rho$ e $f: U \rightarrow Q$ é qualquer (suave, obviamente).

Definição 2.3.1. Sejam A uma conexão principal em $P$ e $\rho_{Q}(\varphi, f): U \rightarrow P \times_{G} Q$ uma seção. Para $x \in U$, a derivada covariante de $\rho_{Q}(\varphi, f)$, com relação a $A$, no ponto $x$, é o jato de primeira ordem, calculado em $x$, da aplicação

$$
D_{s \varphi f x}=\rho_{Q}\left(s(x), \delta_{Q}\left(s, \rho_{Q}(\varphi, f)\right)\right)=\rho_{Q}(s(x), l(\delta(s, \varphi), f))
$$

onde $s: U \rightarrow P$ é uma seção tal que $j_{x} s$ seja representante de $A(x)$. Abreviando $\psi=\rho_{Q}(\varphi, f)$, denotaremos esta operação por

$$
D_{A}(j \psi)(x)=j_{x}\left(D_{s \varphi f x}\right)
$$

Temos então a seguinte proposição.

Proposição 2.3.2. O objeto $D$, da definição anterior, pode ser visto como uma projeção $D: C P \pi_{\pi_{C P}} \times_{\pi_{J P Q}} J\left(P \times_{G} Q\right) \rightarrow \vec{J}\left(P \times_{G} Q\right)$, onde $\pi_{J P Q}: J\left(P \times_{G} Q\right) \rightarrow M$ é a projeção dos jatos a $M$.

Demonstração. Daremos aqui apenas um esboço da demonstração. Os detalhes podem ser encontrados em [25]. Em primeiro lugar, o domínio de $D$ está claramente correto. Tomemos $\left(j_{x} s, j_{x} \rho_{Q}(\varphi, f)\right)$, representantes de $\left(A(x), j_{x} \psi\right) \in C P_{\pi_{C P}} \times_{\pi_{J P Q}}\left(P \times_{G} Q\right)$. Então,

$$
\begin{aligned}
& T_{\psi(x)} \pi_{Q} \circ D_{A}(j \psi)(x)=T_{\psi(x)} \pi_{Q} \circ j_{x}\left(\rho_{Q}(s(x), l(\delta(s, \varphi), f))\right)= \\
& =T_{\psi(x)} \pi_{Q} \circ T_{(p, q)} \rho_{Q}\left(0_{p}, T_{x}(l(\delta(s, \varphi), f))\right)= \\
& =T_{s(x)} \rho \circ T_{(p, q)}\langle P, Q\rangle_{1}\left(0_{p}, T_{x}(l(\delta(s, \varphi), f))\right)= \\
& =0_{x}
\end{aligned}
$$

donde $D_{A}(j \psi)(x) \in \operatorname{ker}\left(T_{\psi(x)} \pi_{Q}\right)$, que mostra que $D_{A}(j \psi)(x) \in \vec{J}\left(P \times_{G} Q\right)$. 
Por outro lado, tomemos $\vec{j}_{x} \rho_{Q}(\psi, f) \in \vec{J}\left(P \times_{G} Q\right)$. Podemos então considerar um elemento $\rho_{Q}(\psi, f): U \rightarrow P \times_{G} Q$, que seja vertical. Tomemos uma seção qualquer $\varphi: U \rightarrow P$ (ajustando os domínios, se necessário). Note que $\rho_{Q}(\varphi, f)$ é seção de $\rho$. Definamos $s: U \rightarrow P$, por

$$
s=r(\varphi, \delta(\psi, \psi(x)))
$$

que está bem definida pelo fato de ser $\rho_{Q}(\psi, f)$ vertical, dado que isto é o mesmo que dizer que $\rho \psi(y)=\rho \psi(x)$, para todo $y \in U$. Com isto,

$$
\rho s=\rho r(\varphi, \delta(\psi, \psi(x)))=\rho \varphi=i d_{U}
$$

cujo jato de primeira ordem pode ser visto como um representante de um elemento em $C P$. Agora, basta ver que

$$
\begin{aligned}
& D_{s \varphi f x}=\rho_{Q}(s(x), l(\delta(s, \varphi), f))=\rho_{Q}(\psi(x), l(\delta(r(\varphi, \delta(\psi, \psi(x))), \varphi), f))= \\
& =\rho_{Q}(\psi(x), l(i \delta(\varphi, r(\varphi, \delta(\psi, \psi(x)))), f))=\rho_{Q}(\psi(x), l(\delta(\psi(x), \psi), f))= \\
& =\rho_{Q}(\psi, f)
\end{aligned}
$$

onde usamos as propriedades $i i$ ) e iii) da proposição 2.3 .1 e as propriedades de $\rho_{Q}$, segundo as ações $r$ e $l$. Ao tomar-se a derivada da última equação, tem-se a sobrejetividade. Para ver que é submersão, consulte [25].

Analisemos com mais detalhes as estruturas do grupoide de calibre de $P$ e de sua ação nos fibrados associados $P \times_{G} Q$, delineadas no início da seção 2.1.

Dado um fibrado principal $(P, M, \rho, G)$, o espaço total do grupoide de calibre é o espaço total do fibrado associado $\left(P \times{ }_{G} P\right)$, onde a ação à esquerda é dada pela inversa transposta da direita, ou seja, $l(g, p)=r(p, i(g))$. As aplicações estruturais do grupoide são então descritas, em elementos, como

$$
\begin{aligned}
& \sigma \rho_{P}\left(p^{\prime}, p\right)=\rho(p) \\
& \tau \rho_{P}\left(p^{\prime}, p\right)=\rho\left(p^{\prime}\right) \\
& \mu\left(\rho_{P}\left(p^{\prime \prime \prime}, p^{\prime \prime}\right), \rho_{P}\left(p^{\prime}, p\right)\right)=\rho_{P}\left(r\left(p^{\prime \prime \prime}, \delta\left(p^{\prime \prime}, p^{\prime}\right)\right), p\right) \\
& \varepsilon \rho(p)=\rho_{P}(p, p) \\
& \iota \rho_{P}\left(p^{\prime}, p\right)=\rho_{P}\left(p, p^{\prime}\right)
\end{aligned}
$$

Suponha agora que $P \times{ }_{G} Q$ seja um fibrado associado a $P$. Definamos $\Phi:\left(P \times{ }_{G} P\right){ }_{\sigma} \times{ }_{\pi_{Q}}\left(P \times{ }_{G} Q\right) \rightarrow$ $P \times_{G} Q$, em elementos, por

$$
\Phi\left(\rho_{P}\left(p^{\prime \prime}, p^{\prime}\right), \rho_{Q}(p, q)\right)=\rho_{Q}\left(r\left(p^{\prime \prime}, \delta\left(p^{\prime}, p\right)\right), q\right)=\rho_{Q}\left(p^{\prime \prime}, l\left(\delta\left(p^{\prime}, p\right), q\right)\right)
$$

Não é difícil verificar que $\Phi$ satisfaz todas as propriedades de ação de um grupoide de Lie em um fibrado. Para obter prolongamentos destes objetos, é útil um estudo sobre as bisseções dos grupoides envolvidos. Como nosso interesse é no comportamento dos jatos destas aplicações, basta que analisemos o que ocorre com seus germes. Assim, sempre que escolhermos domínios "compatíveis", o leitor deve ter em mente que uma restrição a abertos pode ter sido feita. Isto não causa problemas, desde que façamos sempre uma quantidade finita de tais restrições.

Proposição 2.3.3. Existe uma bijeção entre $\operatorname{Aut}_{l o c}(P)$, os automorfismos locais do fibrado principal $P$, e $\operatorname{Bis}_{l o c}\left(P \times_{G} P\right)$, as bisseções locais do grupoide de calibre de $P$.

Demonstração. Considere um automorfismo local de $P$, ou seja, um par de difeomorfismos locais $\left(\phi_{P}, \phi_{M}\right)$, com $\phi_{M}: U \rightarrow V$, e $\phi_{P}:\left.\left.P\right|_{U} \rightarrow P\right|_{V}$, tais que $\rho \phi_{P}=\phi_{M} \rho$ e, para quaisquer $\left.p \in P\right|_{U}$, $g \in G$, valha

$$
\phi_{P} r(p, g)=r\left(\phi_{P}(p), g\right)
$$


Considere uma seção $\beta^{2}: U \rightarrow P, U$ aberto em $M$. Definamos $\beta^{1}: U \rightarrow P$, por $\beta^{1}=\phi_{P} \beta^{2}$ e $b=\rho_{P}\left(\beta^{1}, \beta^{2}\right)$. Claramente, se $\left(\tilde{\beta}^{1}, \tilde{\beta}^{2}\right)$ for uma outra escolha como esta, então

$$
\rho_{P}\left(\tilde{\beta}^{1}, \tilde{\beta}^{2}\right)=\rho_{P}\left(r\left(\beta^{1}, \delta\left(\beta^{1}, \tilde{\beta}^{1}\right)\right), r\left(\beta^{2}, \delta\left(\beta^{2}, \tilde{\beta}^{2}\right)\right)\right)=\rho_{P}\left(\beta^{1}, \beta^{2}\right)
$$

pois, para qualquer função $\theta: U \rightarrow G$,

$$
\delta\left(\beta^{1}, \theta\right)=\delta\left(\phi_{P} \beta^{2}, \theta\right)=\delta\left(\beta^{2}, \theta\right)
$$

visto que $\phi_{P}$ preserva $r$. A relação anterior também é importante para mostrar que, caso $U$ seja um aberto de $M$ que não esteja contido em carta principal alguma, sua descrição local ainda é possível, com escolhas compatíveis. Agora, note que

$$
\sigma \rho_{P}\left(\beta^{1}, \beta^{2}\right)=\rho\langle P, P\rangle_{2}\left(\beta^{1}, \beta^{2}\right)=\rho \beta^{2}=i d_{U}
$$

e também,

$$
\tau \rho_{P}\left(\beta^{1}, \beta^{2}\right)=\rho\langle P, P\rangle_{1}\left(\beta^{1}, \beta^{2}\right)=\rho \beta^{1}=\rho \phi_{P} \beta^{2}=\phi_{M} \rho \beta^{2}=\phi_{M}
$$

mostrando que $b=\rho_{P}\left(\beta^{1}, \beta^{2}\right)$ é bisseção local.

Por outro lado, tomemos $b \in \operatorname{Bis}_{l o c}\left(P \times_{G} P\right)$. Localmente, podemos escrever $b=\rho_{P}\left(\beta^{1}, \beta^{2}\right)$, onde $\beta^{2}: U \rightarrow P$ é uma seção de $\rho$. Definamos $\left(\phi_{P}, \phi_{M}\right)$, por

$$
\phi_{P}=r\left(\beta^{1} \rho, \delta\left(\beta^{2} \rho, i d_{P_{U}}\right)\right)
$$

onde $P_{U}=\rho^{-1}(U)$, e

$$
\phi_{M}=\rho \beta^{1}
$$

Claramente, $\phi_{M}$ não depende das escolhas de representantes e é difeomorfismo local de $M$. Também, $\phi_{P}$ não depende das escolhas de representantes e é $G$-equivariante (ou seja, preserva $r$ ), por construção. Além disso,

$$
\rho \phi_{P}=\rho r\left(\beta^{1} \rho, \delta\left(\beta^{2} \rho, i d_{P_{U}}\right)\right)=\rho \beta^{1} \rho=\phi_{M} \rho
$$

Para ver que $\left(\phi_{P}, \phi_{M}\right)$ se trata de um automorfismo, basta notar que possui inversa $\left(\psi_{P}, \psi_{M}\right)$, dada localmente, por

$$
\psi_{P}=r\left(\beta^{2}\left(\rho \beta^{1}\right)^{-1} \rho, \delta\left(\beta^{1}\left(\rho \beta^{1}\right)^{-1} \rho, i d_{P_{V}}\right)\right)
$$

onde $P_{V}=\left.P\right|_{V}$, com $V=\rho \beta^{1}(U)$, e

$$
\psi_{M}=\left(\rho \beta^{1}\right)^{-1}
$$

como era de se esperar. A demonstração de que se trata de uma inversa é um cálculo direto e será omitida. Isto conclui a bijeção proposta.

Corolário 2.3.1. $J_{b}\left(P \times_{G} P\right)$ age em $C P$.

Demonstração. Tomemos $j_{x} b \in J_{b}\left(P \times_{G} P\right)$ e $A_{x}=\rho_{J P} j_{x} s$, onde $b=\rho_{P}\left(\beta^{1}, \beta^{2}\right)$ e $s: U \rightarrow P$ é um representante adequado (portanto, seção de $\rho$ ). Em termos de seções, a ação é dada por

$$
\tilde{s}=\phi_{P} s \phi_{M}^{-1}=r\left(\beta^{1}, \delta\left(\beta^{2}, s\right)\right)\left(\rho \beta^{1}\right)^{-1}
$$

É claro que, se $t=r(s, g)$, para $g \in G$, então $\tilde{t}=r(\tilde{s}, g)$, donde a transformação é bem definida nos representantes de $A_{x}$. Tomando jatos de primeira ordem, ficamos com $\Phi_{C P}: J_{b}\left(P \times_{G} P\right)_{\sigma_{J P P^{-1}}} \times_{\pi_{C P}} C P \rightarrow C P$, que satisfaz todas as condições de ação de grupoide de Lie em um fibrado.

Observação 2.3.1. Uma outra maneira de construir a ação acima é a seguinte. Em [25], os autores descrevem o prolongamento principal do fibrado principal $P$, denotado por $\left(W P, \rho \circ \pi_{0, P}^{1}, M, W_{m} G\right)$, onde $W P$ pode ser identificado com $J P_{\pi_{J P}} \times_{\pi_{F r}} \operatorname{Fr}(M)$, sendo $\operatorname{Fr}(M)$ o fibrado dos referenciais em $M$, e $W_{m} G$ é o prolongamento principal do grupo de Lie $G$, dado por jatos de automorfismos 
do fibrado principal trivial $\mathbb{R}^{m} \times G$, com $m=\operatorname{dim}(M)$, que fixam a origem de $\mathbb{R}^{m}$, calculados em $(0, e)$. Como mostrado lá, $W P$ tem estrutura de fibrado principal sobre $M$, com grupo estrutural $W_{m} G$. O leitor poderá verificar (com algum esforço) que $J_{b}\left(P \times_{G} P\right)$ é o grupoide de calibre de $W P$, ou seja, é isomorfo a $W P \times_{W G} W P$. Nesta mesma referência, os autores mostram que o fibrado $C P$ tem estrutura de fibrado associado a $W P$. A ação descrita acima é então a ação de grupoide de calibre, canonicamente definida por qualquer fibrado associado a um fibrado principal. Obviamente, para esta apresentação, escolhemos o caminho mais curto da proposição anterior. $\diamond$

A escolha de se trabalhar, sempre que possível, com germes de aplicações, ao invés de, diretamente, com jatos, tem a vantagem de ser imediatamente transponível para objetos de "ordem superior", como jatos de ordem $r$ e conexões de ordem $r$. Apesar de não utilizarmos tais conceitos aqui, o leitor que desejar, poderá fazê-lo sem muito esforço.

Vamos a mais uma ação que nos será útil. Recordemos que, como $P \times_{G} P$ é grupoide de Lie transitivo, está bem definido o grupoide de Lie $\left(P \times_{G} P\right) \alpha_{P P^{-1}} \times_{\alpha_{T M}} G L(T M)$ (ver proposição 2.1.5).

Proposição 2.3.4. O grupoide de Lie $\left(P \times_{G} P\right){ }_{\alpha_{P P^{-1}}} \times_{\alpha_{T M}} G L(T M)$ age sobre $\vec{J}\left(P \times_{G} Q\right)$.

Demonstração. Primeiro, note que, se $\rho_{Q}(\psi, f): U \rightarrow P \times_{G} Q$ for uma seção vertical, para um elemento fixado $\rho_{P}\left(p^{\prime}, p\right)$, com $\rho(p)=\rho \psi(x)$, a ação natural $\Phi:\left(P \times{ }_{G} P\right){ }_{\sigma} \times{ }_{\pi_{Q}}\left(P \times{ }_{G} Q\right) \rightarrow P \times{ }_{G} Q$ induz uma aplicação $\Phi_{\rho_{P}\left(p^{\prime}, p\right)}$, que leva $\rho_{Q}(\psi, f)$ em outra aplicação vertical, sobre $p^{\prime}$, assim:

$$
\Phi_{\rho_{P}\left(p^{\prime}, p\right)} \rho_{Q}(\psi, f)=\Phi\left(\rho_{P}\left(p^{\prime}, p\right), \rho_{Q}(\psi, f)\right)=\rho_{Q}\left(r\left(p^{\prime}, \delta(p, \psi)\right), f\right)=\rho_{Q}\left(p^{\prime}, l(\delta(p, \psi), f)\right)
$$

Com isto em mente, definamos $\Phi_{\lambda}:\left(P \times{ }_{G} P\right){ }_{\alpha_{P P^{-1}}} \times_{\alpha_{T M}} G L(T M){ }_{\sigma} \times{ }_{\pi_{J P Q}} \vec{J}\left(P \times{ }_{G} Q\right) \rightarrow \vec{J}\left(P \times{ }_{G} Q\right)$ como o jato de primeira ordem da aplicação

$$
\Phi_{\rho_{P}\left(p^{\prime}, p\right)} \rho_{Q}(\psi, f) \phi_{M}^{-1}=\rho_{Q}\left(p^{\prime}, l(\delta(p, \psi), f)\right) \phi_{M}^{-1}
$$

onde $j_{x} \rho_{Q}(\psi, f) \in \vec{J}\left(P \times_{G} Q\right), \rho_{P}\left(p^{\prime}, p\right) \in P \times_{G} P$ e $\phi_{M}$ é um difeomorfismo local, tal que $T_{x} \phi_{M} \in$ $G L(T M)$, com domínio sobre $\rho(p)$ e codomínio sobre $\rho\left(p^{\prime}\right)$. As propriedades de ação são cálculos diretos.

Como $J_{b}\left(P \times{ }_{G} P\right)$ age sobre $C P$ e sobre $J\left(P \times{ }_{G} Q\right)$ (ver observação 2.1.6), podemos juntá-las em uma única ação $\Phi_{J C P Q}: J_{b}\left(P \times_{G} P\right)_{\sigma_{J}} \times_{\pi_{C P}} C P_{\pi_{C P}} \times_{\pi_{J P Q}} J\left(P \times_{G} Q\right) \rightarrow C P{ }_{\pi_{C P}} \times_{\pi_{J P Q}} J\left(P \times_{G} Q\right)$. Relembrando ainda o morfismo de grupoides de Lie $\lambda: J_{b}\left(P \times_{G} P\right) \rightarrow\left(P \times_{G} P\right){ }_{\alpha_{P P}-1} \times_{\alpha_{T M}} G L(T M)$ (ver proposição 2.1.5), estamos aptos a enunciar o seguinte teorema.

Teorema 2.3.1. É comutativo, o seguinte diagrama:

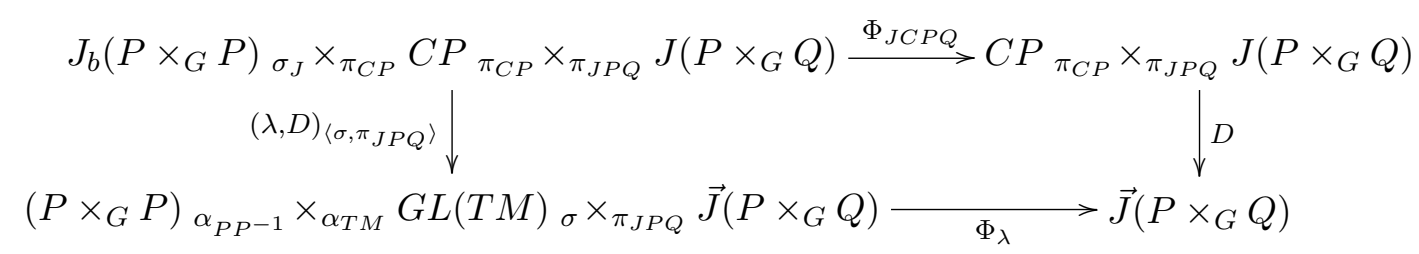

Demonstração. Raciocinemos em termos de (germes de) aplicações. Se $\left(j_{x} b, A_{x}, j_{x} \psi\right)$ for um elemento de $J_{b}\left(P \times_{G} P\right)_{\sigma_{J}} \times_{\pi_{C P}} C P{ }_{\pi_{C P}} \times_{\pi_{J P Q}} J\left(P \times_{G} Q\right)$, consideremos as correspondentes aplicações $b=\rho_{P}\left(\beta^{1}, \beta^{2}\right), s: U \rightarrow P, \psi=\rho_{Q}(\varphi, f)$, onde $s$ e $\varphi$ são seções de $\rho$ e todas têm domínio comum, $U$, com $x \in U$. Chamemos $y=\tau b(x)=\rho \beta^{1}(x)$. Então, por um lado,

$$
\begin{aligned}
& D_{\Phi y} \Phi_{\mathcal{G} C P Q}\left(\rho_{P}\left(\beta^{1}, \beta^{2}\right), s, \rho_{Q}(\varphi, f)\right)= \\
& =D_{\Phi y}\left(r\left(\beta^{1}, \delta\left(\beta^{2}, s\right)\right), \rho_{Q}\left(r\left(\beta^{1}, \delta\left(\beta^{2}, \varphi\right)\right), f\right)\right)\left(\rho \beta^{1}\right)^{-1}= \\
& =\rho_{Q}\left(r\left(\beta^{1}(x), \delta\left(\beta^{2}(x), s(x)\right)\right), l\left(\delta\left(r\left(\beta^{1}, \delta\left(\beta^{2}, s\right)\right), r\left(\beta^{1}, \delta\left(\beta^{2}, \varphi\right)\right)\right), f\right)\right)\left(\rho \beta^{1}\right)^{-1}= \\
& =\rho_{Q}\left(r\left(\beta^{1}(x), \delta\left(\beta^{2}(x), s(x)\right)\right), l(\delta(s, \varphi), f)\right)\left(\rho \beta^{1}\right)^{-1}
\end{aligned}
$$


onde usamos a propriedade $v$ ) da proposição 2.3.1. Por outro lado (usando, eventualmente, os mesmos símbolos para as aplicações em seus germes),

$$
\begin{aligned}
& \Phi_{\lambda}\left(\lambda, D_{s \varphi f x}\right)\left(\rho_{P}\left(\beta^{1}, \beta^{2}\right), s, \rho_{Q}(\varphi, f)\right)= \\
& =\Phi_{\lambda}\left(\left(\rho_{P}\left(\beta^{1}(x), \beta^{2}(x)\right), \rho \beta^{1}\right), \rho_{Q}\left(s(x), \delta_{Q}\left(s, \rho_{Q}(\varphi, f)\right)\right)\right)= \\
& =\rho_{Q}\left(\beta^{1}(x), l\left(\delta\left(\beta^{2}(x), s(x)\right), \delta_{Q}\left(s, \rho_{Q}(\varphi, f)\right)\right)\right)\left(\rho \beta^{1}\right)^{-1}= \\
& =\rho_{Q}\left(r\left(\beta^{1}(x), \delta\left(\beta^{2}(x), s(x)\right)\right), \delta_{Q}\left(s, \rho_{Q}(\varphi, f)\right)\right)\left(\rho \beta^{1}\right)^{-1}= \\
& =\rho_{Q}\left(r\left(\beta^{1}(x), \delta\left(\beta^{2}(x), s(x)\right)\right), l(\delta(s, \varphi), f)\right)\left(\rho \beta^{1}\right)^{-1}
\end{aligned}
$$

o que mostra que, em germes,

$$
D_{\Phi y} \Phi_{\mathcal{G} C P Q}\left(\rho_{P}\left(\beta^{1}, \beta^{2}\right), s, \rho_{Q}(\varphi, f)\right)=\Phi_{\lambda}\left(\lambda, D_{s \varphi f x}\right)\left(\rho_{P}\left(\beta^{1}, \beta^{2}\right), s, \rho_{Q}(\varphi, f)\right)
$$

Tomando jatos de primeira ordem, ficamos com

$$
D \Phi_{J C P Q}\left(j_{x} b, A_{x}, j_{x} \psi\right)=\Phi_{\lambda}\left(\lambda\left(j_{x} b\right), D_{A}(j \psi)(x)\right)
$$

Da arbitrariedade dos elementos, segue a comutatividade do diagrama.

O teorema anterior nos será útil quando discutirmos o conceito de "acoplamento mínimo" que, contrariamente ao caso de ações de grupos, no qual o diagrama em si já seria suficiente ${ }^{10}$, aqui há a necessidade de uma análise adicional, referente às bisseções dos grupoides de ação envolvidos. Até onde pudemos verificar, a discussão da compatibilidade de bisseções, crucial para o entendimento de simetrias mistas (no espaço-tempo e nas fibras, simultaneamente), é feita aqui pela primeira vez.

Definição 2.3.2. Sejam $B \in \operatorname{Bis}\left(J_{b}\left(P \times_{G} P\right)_{\sigma_{J}} \times_{\pi_{C P}} C P{ }_{\pi_{C P}} \times_{\pi_{J P Q}} J\left(P \times_{G} Q\right)\right) e$ $\bar{B} \in \operatorname{Bis}\left(\left(P \times_{G} P\right) \alpha_{\alpha_{P P^{-1}}} \times_{\alpha_{T M}} G L(T M)_{\sigma} \times_{\pi_{J P Q}} \vec{J}\left(P \times_{G} Q\right)\right)$. Dizemos que o par $(B, \bar{B})$ é um par de bisseções covariantemente compativeis, ou que $B$ e $\bar{B}$ são compativeis, quando

$$
(\lambda, D) B=\bar{B} D
$$

Teorema 2.3.2. O grupo $\operatorname{Bis}\left(J_{b}\left(P \times_{G} P\right){ }_{\sigma_{J}} \times_{\pi_{C P}} C P{\pi_{C P}}_{\pi_{J P Q}} J\left(P \times_{G} Q\right)\right)$ se projeta sobre o subgrupo de $\operatorname{Bis}\left(\left(P \times_{G} P\right) \alpha_{P P^{-1}} \times_{\alpha_{T M}} G L(T M)_{\sigma} \times_{\pi_{J P Q}} \vec{J}\left(P \times_{G} Q\right)\right)$ das bisseções que têm imagem contida na imagem de $(\lambda, D)$, de maneira compativel.

Demonstração. Seja $B \in \operatorname{Bis}\left(J_{b}\left(P \times_{G} P\right){ }_{\sigma_{J}} \times_{\pi_{C P}} C P{ }_{\pi_{C P}} \times_{\pi_{J P Q}} J\left(P \times_{G} Q\right)\right)$. Como $D$ é projeção, podemos definir $\bar{B}$ da seguinte maneira. Para $j_{x} \rho_{Q}(\psi, f) \in \vec{J}\left(P \times_{G} Q\right)$, em germes, temos que existem seções $s$ e $\varphi$ de $\rho$, tais que

$$
D_{x}\left(s, \rho_{Q}(\varphi, f)\right)=D_{s \varphi f x}=\rho_{Q}(\psi, f)
$$

Então, em germes (e denotando as aplicações em germes pelos mesmos símbolos),

$$
\begin{aligned}
\bar{B}\left(\rho_{Q}(\psi, f)\right)=\bar{B}\left(D_{x}\left(s, \rho_{Q}(\varphi, f)\right)\right)=\left(\lambda_{x}, D_{x}\right) B\left(s, \rho_{Q}(\varphi, f)\right)= \\
=\left(\lambda_{x}, D_{x}\right)\left(\rho_{P}\left(\beta^{1}, \beta^{2}\right),\left(s, \rho_{Q}(\varphi, f)\right)\right)=\left(\left(\rho_{P}\left(\beta^{1}(x), \beta^{2}(x)\right), \rho \beta^{1}\right), \rho_{Q}(\psi, f)\right)
\end{aligned}
$$

que não depende das escolhas (simultâneas) de $s$ e $\varphi$ e claramente faz com que $\bar{B}$ (definida em jatos), seja bisseção. Ainda, não é difícil ver que

$$
\bar{B} D=(\lambda, D) B
$$

\footnotetext{
${ }^{10} \mathrm{Ou}$ mesmo de fibrados de grupos, como pode ser visto em [13].
} 
Por outro lado, seja $\bar{B} \in \operatorname{Bis}\left(\left(P \times_{G} P\right){ }_{\alpha_{P P^{-1}}} \times_{\alpha_{T M}} G L(T M){ }_{\sigma} \times_{\pi_{J P Q}} \vec{J}\left(P \times_{G} Q\right)\right)$, tal que a imagem de $\bar{B}$ esteja contida na imagem de $(\lambda, D)$. Digamos que, em germes (e em torno de $x \in M$ ),

$$
\bar{B} \rho_{Q}(\psi, f)=\left(\left(\rho_{P}\left(p^{\prime}, p\right), \phi_{M}\right), \rho_{Q}(\psi, f)\right)
$$

lembrando que $\rho_{Q}(\psi, f)$ é vertical. Escolhamos então uma seção $\varphi: U \rightarrow P$, tal que $\varphi(x)=p$, e um automorfismo $\phi_{P}: P \rightarrow P$, tal que $\phi_{P}(p)=p^{\prime}$ e que (onde definida) recubra $\phi_{M}$, o que é possível, visto que $\phi_{M}$ é germe de aplicação cuja derivada primeira está na imagem de $\lambda$. Com isto, construímos a bisseção (local)

$$
b^{\phi}=\rho_{P}\left(\phi_{P} \varphi, \varphi\right)
$$

Ainda, para esta $\varphi$, existe uma seção $s$ de $\rho$, com $D_{x}\left(s, \rho_{Q}(\varphi, f)\right)=\rho_{Q}(\psi, f)$. Então, definamos

$$
B^{\phi}\left(s, \rho_{Q}(\varphi, f)\right)=\left(b^{\phi}, s, \rho_{Q}(\varphi, f)\right)
$$

que é claramente germe de bisseção, para cada escolha de $\phi_{P}$. Ainda,

$$
\begin{aligned}
(\lambda, D)_{x} B^{\phi}\left(s, \rho_{Q}(\varphi, f)\right)=\left(\lambda_{x} \rho_{P}\left(\phi_{P} \varphi, \varphi\right)\right. & \left., D_{x}\left(s, \rho_{Q}(\varphi, f)\right)\right)= \\
= & \left(\left(\rho_{P}\left(p^{\prime}, p\right), \phi_{M}\right), D_{x}\left(s, \rho_{Q}(\varphi, f)\right)\right)=\bar{B} D_{x}\left(s, \rho_{Q}(\varphi, f)\right)
\end{aligned}
$$

donde vemos que

$$
(\lambda, D) B^{\phi}=\bar{B} D
$$

Em outras palavras, a partir de uma tal bisseção $\bar{B}$, podemos construir uma família de bisseções $B^{\phi}$, todas compatíveis com $\bar{B}$.

Observação 2.3.2. Em um primeiro momento, a restrição de que as bisseções $\bar{B}$, do teorema anterior, tenham imagem contida na imagem de $(\lambda, D)$ pode parecer forte. Entretanto, em nossas aplicações, estaremos interessados em formas diferenciais em $\vec{J}\left(P \times_{G} Q\right)$ que sejam invariantes por bisseções que respeitem a estrutura do grupoide $P \times_{G} P$ (e seus descendentes), de modo que é esperada uma restrição de tais bisseções ao menos àquelas oriundas de bisseções em $P \times_{G} P$. Se o subgrupo das bisseções holônomas com relação à forma diferencial em questão for suficientemente bem comportado, definirá um subgrupoide de Lie de $G L(T M)$, em termos de pseudogrupos de Lie de transformações, o que garantiria uma restrição suave da construção envolvida no teorema anterior, fornecendo um subgrupo de bisseções de $J_{b}\left(P \times_{G} P\right){ }_{\sigma_{J}} \times_{\pi_{C P}} C P{ }_{\pi_{C P}} \times_{\pi_{J P Q}} J\left(P \times_{G} Q\right)$, compatível com o anterior. E esta é a situação que geralmente temos em mãos. Para um tratamento de questões sobre holonomia de formas diferenciais, subgrupos de bisseções holônomas e sua relação com pseudogrupos de Lie de transformações, veja [36]. $\diamond$

Enfim, é conveniente uma discussão sobre o comportamento da curvatura de conexões principais, mediante a ação do grupoide de calibre.

Em primeiro lugar, note que $C P$ é fibrado afim sobre $M$, modelado sobre o fibrado vetorial $T^{*} M \otimes\left(P \times_{G} \mathfrak{g}\right.$ ), onde $\mathfrak{g}$ denota a álgebra de Lie de $G$ (veja [25]). O fibrado $P \times_{G} \mathfrak{g}$, sendo fibrado associado a $P$, fornece a ação $\Phi_{\mathfrak{g}}:\left(P \times_{G} P\right)_{\sigma} \times_{\pi_{\mathfrak{g}}}\left(P \times_{G} \mathfrak{g}\right) \rightarrow P \times_{G} \mathfrak{g}$ dada, em elementos, por

$$
\Phi_{\mathfrak{g}}\left(\rho_{P}\left(p^{\prime \prime}, p^{\prime}\right), \rho_{\mathfrak{g}}(p, \xi)\right)=\rho_{\mathfrak{g}}\left(r\left(p^{\prime \prime}, \delta\left(p^{\prime}, p\right)\right), \xi\right)=\rho_{\mathfrak{g}}\left(p^{\prime \prime}, \operatorname{ad}\left(\delta\left(p, p^{\prime}\right)\right)(\xi)\right)
$$

onde ad é a derivada de Ad, a representação adjunta, ação à esquerda de $G$ em $\mathfrak{g}$ que fornece a estrutura de fibrado associado a $P \times_{G} \mathfrak{g}$. Também, do fato de $J_{b}\left(P \times_{G} P\right)$ ser um grupoide de Lie que age sobre $C P$, o prolongamento de primeira ordem desta ação (confira a observação 2.1.7) fornece a ação $\Phi_{J J C P}: J_{b} J_{b}\left(P \times_{G} P\right)_{\sigma_{J J}} \times_{\pi_{J C P}} J(C P) \rightarrow J(C P)$. Temos ainda o morfismo de grupoides óbvio $\lambda^{2}: J_{b} J_{b}\left(P \times_{G} P\right) \rightarrow P \times_{G} P{ }_{\alpha_{P P^{-1}}} \times_{\alpha_{T M}} G L(T M)$. Como último ingrediente, a curvatura pode ser vista como um operador (morfismo estrito de fibrados) $R: J(C P) \rightarrow\left(P \times_{G} \mathfrak{g}\right) \otimes \bigwedge^{2} T^{*} M$ (confira [25]).

Estamos aptos a enunciar o seguinte teorema. 
Teorema 2.3.3. É comutativo, o seguinte diagrama:

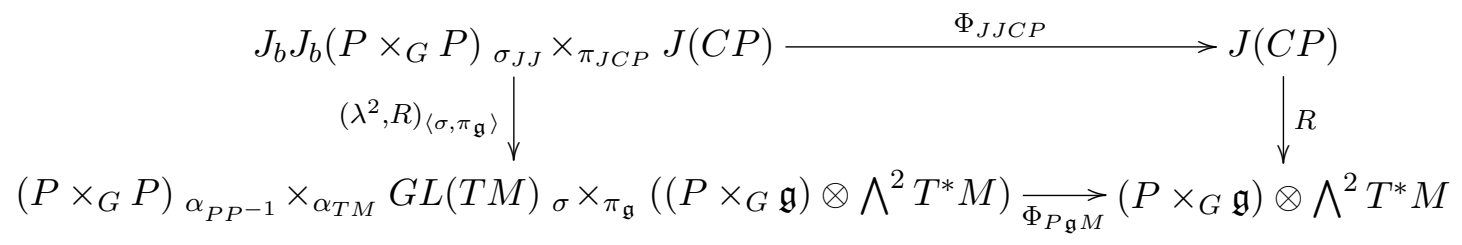

onde $\Phi_{P \mathfrak{g} M}:\left(P \times_{G} P\right)_{\alpha_{P P^{-1}}} \times_{\alpha_{T M}} G L(T M)_{\sigma} \times_{\pi_{\mathfrak{g}}}\left(\left(P \times_{G} \mathfrak{g}\right) \otimes \bigwedge^{2} T^{*} M\right) \rightarrow\left(P \times_{G} \mathfrak{g}\right) \otimes \bigwedge^{2} T^{*} M$ denota a ação conjunta da ação de $G L(T M)$ em $\bigwedge^{2} T^{*} M$ e da ação $\Phi_{\mathfrak{g}}$.

Demonstração. Comecemos por notar que bisseções de $J_{b}\left(P \times_{G} P\right)$ agem sobre conexões principais. De fato, dadas uma bisseção $B: M \rightarrow J_{b}\left(P \times_{G} P\right)$ e uma conexão principal $A: M \rightarrow C P$, podemos escrever, em germes (note que isto vale globalmente, inclusive),

$$
\Phi_{\mathcal{G} J C P}(B, A)=\Phi_{J C P}(B, A)\left(\tau_{J} B\right)^{-1}
$$

Dado $x \in M$, escolhamos representantes $\left(\beta^{1}, \beta^{2}\right)$, tais que $B(x)=j_{x}\left(\rho_{P}\left(\beta^{1}, \beta^{2}\right)\right)$ e $\varphi$, tal que $j_{x} \varphi$ seja representante de $A(x), \operatorname{com} \varphi(x)=\beta^{2}(x)=p$. Assim, em $y=\left(\tau_{J} B\right)(x)$,

$$
\Phi_{\mathcal{G} J C P}(B, A)(y)=\Phi_{J C P}\left(j_{x} b, A_{x}\right)=j_{y}\left(r\left(\beta^{1}, \delta\left(\beta^{2}, \varphi\right)\right)\left(\rho \beta^{1}\right)^{-1}\right)
$$

Com estes dados, podemos construir uma aplicação $\gamma: P \rightarrow J P$, seção de $\pi_{0, P}^{1}, G$-equivariante. Para $p^{\prime} \in P$, tome $y=\rho\left(p^{\prime}\right), x=\left(\tau_{J} B\right)^{-1}(y), \beta^{1}$ um representante alvo de $B(x)$ tal que $\beta^{1}(x)=p^{\prime}$ e $\varphi$ um representante de $A(x)$, tal que $\varphi(x)=\beta^{2}(x)=p$, onde $\beta^{2}$ é o representante fonte de $B(x)$, que acompanha $\beta^{1}$. Então,

$$
\gamma\left(p^{\prime}\right)=j_{y}\left(r\left(\beta^{1}, \delta\left(\beta^{2}, \varphi\right)\right)\left(\rho \beta^{1}\right)^{-1}\right)
$$

É um cálculo direto que $\gamma\left(p^{\prime}\right) \in J_{p^{\prime}} P$. Para mostrar que $\gamma$ é equivariante, considere $\tilde{p}=r\left(p^{\prime}, g\right)$, para algum $g \in G$. Então, $\tilde{y}=y, \tilde{x}=x$ e podemos escolher como representantes de $B(x)$ e $A(x)$, as aplicações $\tilde{\beta}^{1}=r\left(\beta^{1}, g\right), \tilde{\beta}^{2}=r\left(\beta^{2}, g\right)$ e $\tilde{\varphi}=r(\varphi, g)$. Com isto,

$$
\begin{aligned}
& \gamma(\tilde{p})=j_{y}\left(r\left(\tilde{\beta}^{1}, \delta\left(\tilde{\beta}^{2}, \tilde{\varphi}\right)\right)\left(\rho \tilde{\beta}^{1}\right)^{-1}\right)= \\
& =j_{y}\left(r\left(r\left(\beta^{1}, g\right), \delta\left(r\left(\beta^{2}, g\right), r(\varphi, g)\right)\right)\left(\rho \beta^{1}\right)^{-1}\right)= \\
& =j_{y}\left(r\left(\beta^{1}, m\left(g, m\left(m\left(i(g), \delta\left(\beta^{2}, \varphi\right)\right), g\right)\right)\right)\left(\rho \beta^{1}\right)^{-1}\right)= \\
& =j_{y}\left(r\left(\beta^{1}, m\left(\delta\left(\beta^{2}, \varphi\right), g\right)\right)\left(\rho \beta^{1}\right)^{-1}\right)= \\
& =j_{y}\left(r\left(r\left(\beta^{1}, \delta\left(\beta^{2}, \varphi\right)\right)\left(\rho \beta^{1}\right)^{-1}, g\right)\right)
\end{aligned}
$$

o que mostra que $\gamma$ é equivariante e, portanto, induz uma seção de $C P$.

Da expressão de $\gamma$, podemos inferir que $\gamma$ é simplesmente a conexão $A$, transformada pela coleção de automorfismos de $P$ definida, pontualmente, pelas bisseções $B(x)$, para cada $x \in M$. Como a curvatura de $A$ é, por definição, equivariante pela ação de automorfismos de $P$, temos

$$
R_{\gamma\left(p^{\prime}\right)}(\xi, \eta)=R_{T \phi_{P} A}\left(\phi_{P}(p)\right)(\xi, \eta)=T \phi_{P} R_{A}(p)\left(T \phi_{M}^{-1}(\xi), T \phi_{M}^{-1}(\eta)\right)
$$

onde $\left(\phi_{P}, \phi_{M}\right)$ denota o automorfismo local de $P$ definido por $B(x)$. Como $R_{A}(p)\left(T \phi_{M}^{-1}(\xi), T \phi_{M}^{-1}(\eta)\right) \in P \times_{G} \mathfrak{g}$, tal automorfismo é induzido pela ação $\Phi_{\mathfrak{g}}$ que, neste caso, se escreve

$$
\Phi_{\mathfrak{g}}\left(\rho_{P}\left(\beta^{1}(x), \beta^{2}(x)\right), R_{A}\left(\beta^{2}(x)\right)(\xi, \eta)\right)=R_{\gamma}\left(\beta^{1}(x)\right)\left(T_{\beta^{1}(x)} \rho T_{x} \beta^{1}(\xi), T_{\beta^{1}(x)} \rho T_{x} \beta^{1}(\eta)\right)
$$

que é a expressão da ação $\Phi_{P \mathfrak{g} M}$, calculada em $\left(\lambda^{2}, R\right)$. Logo, o diagrama proposto é comutativo.

O diagrama do teorema anterior nos será útil quando discutirmos um teorema de grande importância nas teorias de calibre, conhecido na literatura como o teorema de Utiyama. 
Observação 2.3.3. Uma outra maneira de obter o resultado anterior pode ser elaborada com a seguinte estratégia. Uma conexão principal pode ser vista como uma seção da âncora do algebroide $\mathfrak{A}\left(P \times_{G} P\right)$ (veja [29]), ou seja, uma aplicação $\gamma: T M \rightarrow \mathfrak{A}\left(P \times_{G} P\right)$, tal que $a \gamma=i d_{T M}$. Temos então duas ações: $\Phi_{T M}: J_{b}\left(P \times_{G} P\right)_{\sigma_{J}} \times_{\pi_{M}} T M \rightarrow T M$, dada por

$$
\Phi_{T M}\left(T_{\left(\beta^{1}, \beta^{2}\right)(x)} \rho_{P}\left(T_{x} \beta^{1}, T_{x} \beta^{2}\right), \xi_{x}\right)=\left(T_{\beta^{1}(x)} \rho T_{x} \beta^{1}\right)\left(\xi_{x}\right)
$$

e a ação adjunta Ad: $J_{b}\left(P \times_{G} P\right)_{\sigma_{J}} \times_{\pi_{A}} \mathfrak{A}\left(P \times_{G} P\right) \rightarrow \mathfrak{A}\left(P \times_{G} P\right)$ dada, em germes, por

$$
\operatorname{Ad}\left(\rho_{P}\left(\beta^{1}, \beta^{2}\right), \rho_{P}\left(\mathrm{Fl}_{t}^{\xi}, p\right)\right)=\rho_{P}\left(r\left(\beta^{1} \rho, \delta\left(\beta^{2} \rho, i d_{P_{U}}\right)\right), \beta^{1}(x)\right) \mathrm{Fl}_{t}^{\xi}
$$

onde $\mathrm{Fl}_{t}^{\xi}$ denota o fluxo do campo $\xi \in \mathfrak{A}\left(P \times{ }_{G} P\right)$, lembrando que tal campo é $\sigma$-vertical e invariante à direita ${ }^{11}$. Com isto, dadas uma bisseção $B: M \rightarrow J_{b}\left(P \times_{G} P\right)$ e uma conexão

$A: T M \rightarrow \mathfrak{A}\left(P \times_{G} P\right)$, fazemos, para cada $x \in M$, com $y=\tau_{J} B(x)$, uma $\gamma_{y}: T_{y} M \rightarrow \mathfrak{A}_{y}\left(P \times_{G} P\right)$, dada por

$$
\gamma_{y}=\operatorname{Ad}(B(x))\left(A(x)\left(\Phi_{T M} B(x)\right)^{-1}\right)
$$

que é uma conexão principal. Esta estratégia, levada a cabo, fornece o mesmo resultado para a curvatura, dado pelo teorema anterior. $\diamond$

Observação 2.3.4. Como o operador curvatura $R$, acima, é uma projeção ${ }^{12}$, um raciocínio análogo ao desenvolvido no teorema 2.3.2 permite concluir que, ao definirmos bisseções compatíveis, utilizando a mesma técnica, teremos um resultado semelhante: o grupo de bisseções de $J_{b} J_{b}\left(P \times_{G} P\right)_{\sigma_{J J}} \times_{\pi_{J C P}} J(C P)$ se projeta sobre o subgrupo das bisseções do grupoide de ação de $\Phi_{P \mathfrak{g} M}$ que têm imagem contida na imagem de $\left(\lambda^{2}, R\right)$, de maneira compatível. $\diamond$

Este é o arsenal que precisaremos para discutir simetrias no exemplo alvo desta tese, a saber, a Teoria Clássica dos Campos.

\footnotetext{
${ }^{11} \mathrm{O}$ leitor poderá encontrar mais detalhes sobre a ação adjunta em [36].

${ }^{12} \mathrm{O}$ leitor poderá encontrar uma demonstração este fato em [37].
} 


\section{Capítulo 3}

\section{Simetrias em teoria clássica de campos, na formulação lagrangiana}

\subsection{Campos}

Grosseiramente falando, uma teoria clássica de campos versa sobre as propriedades de um espaço de seções suaves de um determinado fibrado, dito o fibrado das configurações de campo. Estas seções são ditas "os campos da teoria" e a base do fibrado é, geralmente, o espaço-tempo (ou simplesmente o tempo, no caso da mecânica não autônoma, que pode ser vista como uma teoria de campos). A dinâmica é então especificada por equações diferenciais, as equações do campo, que podem ser expressas utilizando-se várias técnicas, como prolongamentos a jatos de seções, formas diferenciais, etc. Nesta seção, estaremos interessados em teorias de campos lagrangianas, ou seja, teorias de campos descritas por uma forma diferencial adequada, definida nos jatos de seções do fibrado ${ }^{1}$. Tornemos precisos estes conceitos.

De agora em diante, fixemos um fibrado suave geral $(E, M, \pi)$, que será referido como fibrado de configurações, onde a base $M$ é orientável e tem dimensão $m$.

Definição 3.1.1. Uma $k$-forma $\omega \in \Omega^{k}(E)$ é dita horizontal quando, para qualquer $\xi \in V^{\pi} E$, o fibrado vertical de $E$, com relação a $\pi$, tivermos, necessariamente,

$$
i_{\xi} \omega=0
$$

A justificativa para a terminologia anterior é que, se $\omega$ for uma forma horizontal então, localmente, seus coeficientes são funções em $E$, mas seus covetores se restringem a $M$.

Definição 3.1.2. Uma forma lagrangiana, ou ainda, uma densidade lagrangiana, ou simplesmente, uma lagrangiana em $(E, M, \pi)$, de ordem $r$, é uma $m$-forma horizontal $\mathfrak{L} \in \Omega^{m}\left(J^{r} E\right)$, com relação à projeção $\pi \circ \pi_{0, E}^{r}$.

Apesar da generalidade da definição anterior, frequentemente nos restringiremos a lagrangianas de primeira ordem, por simplicidade. O leitor interessado não terá dificuldade em aplicar as técnicas desenvolvidas neste texto a lagrangianas de ordem superior.

A lagrangiana de uma teoria é o ponto de partida para a formulação de uma dinâmica, ditada pelo princípio da ação estacionária. Tal princípio diz que um campo satisfará as equações de movimento do sistema, quando for extremal de um funcional ação. Precisemos isto.

Definição 3.1.3. Seja $\mathfrak{L}$ uma lagrangiana de ordem $r$, em $(E, M, \pi)$. O funcional ação desta lagrangiana é a aplicação $S: \Gamma_{K-l o c}(E) \rightarrow \mathbb{R}$, dada por

$$
S_{K}[\varphi]=\int_{K}\left(j^{r} \varphi\right)^{*} \mathfrak{L}
$$

\footnotetext{
${ }^{1}$ Para maiores informações, o leitor poderá consultar [10].
} 
onde

$$
\Gamma_{K-l o c}(E)=\coprod_{K \in \mathbf{K}} \Gamma_{K}(E)
$$

é a união disjunta dos espaços $\Gamma_{K}(E)$, de seções locais de $\pi$ definidas em vizinhanças que contenham o compacto $K$ em $M$, sendo que $\mathbf{K}$ denota a coleção dos subconjuntos compactos de $M$.

Definição 3.1.4. Fixemos um compacto $K$ em $M$. Seja $X \in V E$, ou seja, $X$ é campo vertical em $E$, de suporte (projetado à base) contido em $K$, tal que $j^{r-1} X$ se anule em $\partial K$ (a fronteira de $K$ ). A um tal $X$, damos o nome de deformação sobre $K$. Denotemos por $\phi_{t}: E \rightarrow E$ a família de automorfismos estritos de $E$, definida pelo fluxo $\mathrm{Fl}_{t}^{X}$ de $X$. Definimos a variação de $S$, em $K$, ao longo de $X$, no ponto $\varphi$, por

$$
\delta_{X} S_{K}[\varphi]=\left.\frac{d}{d t}\left(S_{K}\left[\phi_{t} \circ \varphi\right]\right)\right|_{t=0}
$$

Definição 3.1.5. Denotando o espaço das seções locais de $\pi$ por $\Gamma_{l o c}(E)$, dizemos que uma seção $\varphi \in \Gamma_{\text {loc }}(E)$ é uma solução (clássica), se for um extremal para a ação, ou seja, quando valer

$$
\delta_{X} S_{K}[\varphi]=0
$$

para todo compacto $K$ em $M$, tal que $\varphi \in \Gamma_{K}(E)$, e toda deformação $X$ sobre $K$.

Agora, nos interessa uma formulação da variação de $\mathfrak{L}$, que forneça condições para extremais, em termos de formas diferenciais. Para tanto, são necessários alguns conceitos preliminares.

Definição 3.1.6. Uma 1-forma de contato, de ordem $r$, é uma forma diferencial $\alpha \in \Omega^{1}\left(J^{r} E\right)$, tal que

$$
\left(j^{r} \varphi\right)^{*} \alpha=0
$$

para toda $\varphi \in \Gamma_{\text {loc }}(E)$. Uma forma de contato de ordem $r$ é um elemento do ideal bilateral em $\Omega^{\bullet}\left(J^{r} E\right)$, gerado pelas 1 -formas de contato de mesma ordem.

Observação 3.1.1. Para algumas pessoas, pode ser útil exibirmos expressões em coordenadas para as 1-formas de contato. Tomemos uma carta fibrada em $E$, a qual denotaremos por $\left(x^{\mu}, q^{i}\right)$, para indicar as $m$ funções coordenadas $x^{1}, \ldots, x^{m}$ em um aberto $U$ na base e as $n$ funções coordenadas $q^{1}, \ldots, q^{n}$ em um aberto de $\pi^{-1}(U)$. Tal carta induz uma carta fibrada em $J^{r} E$, que será aqui denotada por $\left(x^{\mu}, q^{i}, q_{\mu}^{i}, \ldots, q_{\mu_{1} \ldots \mu_{r}}^{i}\right)$ (para detalhes veja, por exemplo, [10]). Localmente, uma base para o espaço das 1-formas de contato pode ser expressa por

$$
\begin{aligned}
\alpha^{i} & =d q^{i}-\sum_{\nu=1}^{m} q_{\nu}^{i} d x^{\nu} \\
\alpha_{\mu}^{i} & =d q_{\mu}^{i}-\sum_{\nu=1}^{m} q_{\mu \nu}^{i} d x^{\nu} \\
& \ldots \\
\alpha_{\mu_{1} \ldots \mu_{r}}^{i} & =d q_{\mu_{1} \ldots \mu_{r-1}}^{i}-\sum_{\nu=1}^{m} q_{\mu_{1} \ldots \mu_{r-1} \nu}^{i} d x^{\nu}
\end{aligned}
$$

para $i=1, \ldots, n . \diamond$

Definição 3.1.7. Uma forma de Poincaré-Cartan, de ordem $r$, é uma m-forma diferencial $\theta \in \Omega^{m}\left(J^{2 r-1} E\right)$, que satisfaça

i) para todos $X, Y \in \mathfrak{X}\left(J^{2 r-1} E\right)$, verticais segundo a projeção $\pi \pi_{0, E}^{2 r-1}, i_{X} i_{Y} \theta=0$;

ii) para todo $X \in \mathfrak{X}\left(J^{2 r-1} E\right)$, vertical segundo a projeção $\pi_{r-1, E}^{2 r-1}, i_{X} \theta=0$; 
iii) para todo $X \in \mathfrak{X}\left(J^{2 r-1} E\right)$, vertical segundo a projeção $\pi_{0, E}^{2 r-1}$, $i_{X} d \theta$ é forma de contato.

Observação 3.1.2. Como mostrado em [10], as propriedades que definem formas de PoincaréCartan limitam fortemente seus comportamentos locais. Especificamente, a expressão local de uma forma de Poincaré-Cartan de ordem $r$ consiste da soma de uma $m$-forma horizontal, cujos coeficientes dependem de componentes até ordem $r$, e de uma forma de contato. Assim, uma tal forma pode ser vista como a soma de uma lagrangiana de ordem $r$ e um termo de contato. Para $r=1$, formas de Poincaré-Cartan e lagrangianas estão em relação biunívoca. A expressão em coordenadas locais de uma $\theta_{\mathfrak{L}}$, cuja "parte horizontal" (conceito a ser realizado globalmente mais adiante) seja uma lagrangiana $\mathfrak{L}$ de primeira ordem, é dada da seguinte maneira. Tomemos uma carta fibrada de $E$ denotada, como antes, por $\left(x^{\mu}, q^{i}\right)$. Tal carta induz uma carta fibrada em $J E$, denotada por $\left(x^{\mu}, q^{i}, q_{\mu}^{i}\right)$. Uma lagrangiana se escreve, localmente, como $\mathfrak{L}=L d^{m} x$, onde $d^{m} x=d x^{1} \wedge \ldots \wedge d x^{m}$. Então,

$$
\theta_{\mathfrak{L}}=L d^{m} x+\sum_{i=1}^{n} \sum_{\mu=1}^{m} \frac{\partial L}{\partial q_{\mu}^{i}} \alpha^{i} \wedge d^{m} x_{\mu}
$$

onde $\alpha^{i}$ são as 1-formas de contato

$$
\alpha^{i}=d q^{i}-\sum_{\nu=1}^{m} q_{\nu}^{i} d x^{\nu}
$$

e

$$
d^{m} x_{\mu}=i_{\partial / \partial x^{\mu}} d^{m} x
$$

mostrando que $\theta_{\mathfrak{L}}$ e $\mathfrak{L}$ diferem por uma forma de contato. Um breve rearranjo de termos leva a

$$
\theta_{\mathfrak{L}}=\left(L-\sum_{i=1}^{n} \sum_{\mu=1}^{m} \frac{\partial L}{\partial q_{\mu}^{i}} q_{\mu}^{i}\right) d^{m} x+\sum_{i=1}^{n} \sum_{\mu=1}^{m} \frac{\partial L}{\partial q_{\mu}^{i}} d q^{i} \wedge d^{m} x_{\mu}
$$

Renomeando

$$
\begin{gathered}
p_{i}^{\mu}=\frac{\partial L}{\partial q_{\mu}^{i}} \\
p=L-\sum_{i=1}^{n} \sum_{\mu=1}^{m} \frac{\partial L}{\partial q_{\mu}^{i}} q_{\mu}^{i}
\end{gathered}
$$

ficamos com a sugestiva expressão

$$
\theta_{\mathfrak{L}}=\sum_{i=1}^{n} \sum_{\mu=1}^{m} p_{i}^{\mu} d q^{i} \wedge d^{m} x_{\mu}+p d^{m} x
$$

conhecida da geometria multissimplética (veja, por exemplo, [16]). Para $r=2$, esta unicidade se perde. Em geral, há várias formas de Poincaré-Cartan que admitem a mesma lagrangiana (exceto se $m=1)$. Entretanto, neste caso, é possível escolher canonicamente uma tal forma, dita "reduzida" [10]. Para $r>2($ e $m \neq 1)$, mesmo a escolha de uma forma de Poincaré-Cartan reduzida, associada a uma determinada lagrangiana, envolve a escolha de uma conexão simétrica na base. Assim, temos ambiguidades com as quais devemos lidar, se quisermos utilizar formas de Poincaré-Cartan para descrever propriedades de sistemas lagrangianos. Porém, tal esforço não é vão, devido ao seguinte. Em primeiro lugar, se $\theta_{\mathfrak{L}}$ for uma forma de Poincaré-Cartan associada a $\mathfrak{L}$, elas diferem por um termo de contato. Assim, para qualquer $\varphi \in \Gamma_{l o c}(E)$,

$$
\left(j^{2 r-1} \varphi\right)^{*} \theta_{\mathfrak{L}}=\left(j^{2 r-1} \varphi\right)^{*} \hat{\mathfrak{L}}
$$

onde $\hat{\mathfrak{L}}$ denota o pull-back da lagrangiana $\mathfrak{L}$ de ordem $r$, portanto definida em $J^{r} E$, ao espaço $J^{2 r-1} E$, onde está definida $\theta_{\mathfrak{L}}$, por meio da projeção $\pi_{r, E}^{2 r-1}$. Portanto, têm mesmo comportamento 
mediante o funcional ação, ou seja, dados um compacto $K$ em $M$ e uma seção $\varphi \in \Gamma_{K}(E)$, temos

$$
S_{K}[\varphi]=\int_{K}\left(j^{r} \varphi\right)^{*} \mathfrak{L}=\int_{K}\left(j^{2 r-1} \varphi\right)^{*} \theta_{\mathfrak{L}}
$$

que, do ponto de vista físico, é o objeto central a ser considerado. Em segundo lugar, as interações fundamentais descritas pelo modelo padrão envolvem lagrangianas de primeira ordem, onde não há tal ambiguidade. Mesmo modelos que consideram efeitos de gravitação não ultrapassam ordem dois, onde uma possível ambiguidade pode ser resolvida sem a introdução de objetos adicionais. Em suma, a principal classe de exemplos que nos motiva não necessita de qualquer tratamento adicional para transitarmos entre lagrangianas e formas de Poincaré-Cartan. Entretanto, estas últimas simplificam muito o ferramental referente às soluções das equações de Euler-Lagrange, à descrição do teorema de Noether e até mesmo à relação desta abordagem com a formulação hamiltoniana, a qual não trataremos aqui. Sua utilidade se concretiza ao notarmos que, dada uma lagrangiana $\mathfrak{L}$, há um procedimento para encontrarmos formas de Poincaré-Cartan, associadas a L. $\diamond$

Notação 3.1.1. Se a "parte horizontal" (confira a observação 3.1.3) de $\theta$ for uma lagrangiana $\mathfrak{L}$, dizemos que $\theta$ é forma de Poincaré-Cartan associada a $\mathfrak{L}$ e escrevemos $\theta=\theta_{\mathfrak{L}}$, para indicar esta relação. $\diamond$

Observação 3.1.3. Precisemos o significado de "parte horizontal", nas observação e notação anteriores. O uso das aspas se deve ao fato de que não há uma noção canônica do que seria, globalmente, a parte horizontal de uma tal forma. Em geral, é necessário que esteja definida alguma cisão de $J^{2 r-1} E$ para que isto tenha sentido. O leitor interessado poderá consultar [10] para detalhes. O que nos interessa é que, dada uma lagrangiana $\mathfrak{L}$, podemos definir morfis$\operatorname{mos} \mathcal{E}(\mathfrak{L}): J^{2 r} E \rightarrow V^{*} E \otimes \bigwedge^{m} T^{*} M$, chamado morfismo de Euler-Lagrange, e $\mathcal{K}(\mathfrak{L}): J^{2 r-1} E \rightarrow$ $V^{*}\left(J^{r-1} E\right) \otimes \bigwedge^{m-1} T^{*} M$, chamado morfismo de Poincaré-Cartan, que codificam, respectivamente, as informações sobre as equações de Euler-Lagrange e o termo de bordo da derivada variacional da ação de $\mathfrak{L}$. Embora $\mathcal{E}(\mathfrak{L})$ seja único, $\mathcal{K}(\mathfrak{L})$ não pode ser unicamente determinado para $r \geq 3$. Pode-se obter um $\mathcal{K}(\mathfrak{L})$ canônico, mediante a escolha de uma conexão linear e simétrica em $M$, como mostrado em [24]. Cada escolha de $\mathcal{K}(\mathfrak{L})$ fornece uma forma de Poincaré-Cartan $\theta_{\mathfrak{L}}$, dada por

$$
\theta_{\mathfrak{L}}=\mathcal{K}(\mathfrak{L})+\left(\pi_{r, E}^{2 r-1}\right)^{*} \mathfrak{L}
$$

de modo que, quando contraídas com um campo vertical, $\theta_{\mathfrak{L}}$ e $\mathcal{K}(\mathfrak{L})$ coincidem. Assim, $\theta_{\mathfrak{L}}$ codifica a informação do termo de bordo da derivada variacional de $\mathfrak{L}$. Uma vantagem de lidarmos com formas de Poincaré-Cartan é que esta codifica a informação do termo de bordo da derivada variacional de $\mathfrak{L}$, quando contraída com campos projetáveis em geral e não apenas verticais, o que é essencial para o estudo de simetrias, enquanto que $\mathcal{K}(\mathfrak{L})$ fornece apenas a variação vertical de tais campos. Além disso, ao considerarmos uma variação de $S$, vemos que, após uma pequena álgebra,

$$
\delta_{X} S_{K}[\varphi]=\int_{K}\left(j^{2 r-1} \varphi\right)^{*} i_{j^{2 r-1} X} d \theta_{\mathfrak{L}}+\int_{\partial K}\left(j^{2 r-1} \varphi\right)^{*} i_{j^{2 r-1} X} \theta_{\mathfrak{L}}
$$

É possível então mostrar que $\varphi$ é extremal de $S$ se, e somente se, $\left(j^{2 r-1} \varphi\right)^{*} i_{j^{2 r-1} X} d \theta_{\mathfrak{L}}=0$, para todo campo vetorial vertical $X$ em $E$ (veja [10]). São estas equações que chamaremos equações de Euler-Lagrange para $\mathfrak{L}$. Portanto, enquanto $\theta_{\mathfrak{L}}$ codifica a informação do termo de bordo da derivada variacional de $\mathfrak{L}$, a forma $d \theta_{\mathfrak{L}}$ codifica a informação do termo de Euler-Lagrange. Isso simplifica bastante o tratamento de simetrias. $\diamond$

Como temos formas diferenciais do tipo $\theta_{\mathfrak{L}}$ que descrevem a variação de $\mathfrak{L}$ (lembre que $\theta_{\mathfrak{L}}$ não é, em geral, única), podemos falar em invariância pela ação de um grupoide de Lie e relacionar isto a leis de conservação. Inicialmente, por simplicidade, faremos um tratamento mais estrito do conceito de simetria, deixando a discussão do conceito amplo que, do ponto de vista funcional, é o 
correto, para a seção 3.3. Fixemos uma lagrangiana $\mathfrak{L}$, de ordem $r$, e uma forma de Poincaré-Cartan $\theta_{\mathfrak{L}}$, para esta lagrangiana.

Definição 3.1.8. Um grupoide de simetrias para $\theta_{\mathfrak{L}}$ é um grupoide de Lie $(G, M, \sigma, \tau, \mu, \varepsilon, \iota)$, tal que exista uma ação $\Phi: G_{\sigma} \times_{\pi} E \rightarrow E$, de modo que $\theta_{\mathfrak{L}}$ seja invariante por prolongamentos de ordem $2 r-1$ de bisseções verticalmente constantes de $G_{\sigma} \times_{\pi} E$ (confira definição 2.1.10). Diremos que bisseções de $G$ ou, equivalentemente, bisseções verticalmente constantes de $G_{\sigma} \times_{\pi} E$, são simetrias de $\theta_{\mathfrak{L}}$. As projeções alvo $\Phi B$ de bisseções verticalmente constantes de $G_{\sigma} \times_{\pi} E$, também serão chamadas de simetrias.

Fixemos um grupoide de simetrias para $\theta_{\mathfrak{L}}$, com mesma notação da definição anterior.

Teorema 3.1.1. As simetrias de $\theta_{\mathfrak{L}}$ levam soluções em soluções.

Demonstração. Sejam $\varphi \in \Gamma_{l o c}(E)$ uma solução das equações de Euler-Lagrange e

$B \in \operatorname{Bis}\left(G_{\sigma} \times_{\pi} E\right)$ uma simetria de $\theta_{\mathfrak{L}} . B=\left(b \pi, i d_{E}\right)$ induz um automorfismo de $E$ que leva $\varphi$ em

$$
\tilde{\varphi}=\Phi B \varphi(\tau b)^{-1}
$$

cujo prolongamento de ordem $k=2 r-1$ é

$$
\begin{aligned}
j^{k} \tilde{\varphi} & =j^{k}(\Phi B) j^{k} \varphi j^{k}(\tau b)^{-1}=j^{k} \Phi\left(j^{k} b, j^{k} \varphi\right) j^{k}(\tau b)^{-1}=\Phi_{J^{k}}\left(j^{k} b, j^{k} \varphi\right)= \\
& =\Phi_{J^{k}} j^{k} B j^{k} \varphi
\end{aligned}
$$

Portanto, para uma deformação $X$, temos

$$
\begin{aligned}
\left(j^{k} \tilde{\varphi}\right)^{*} i_{j^{k} X} d \theta_{\mathfrak{L}} & =\left(\Phi_{J^{k}} j^{k} B j^{k} \varphi\right)^{*} i_{j^{k} X} d \theta_{\mathfrak{L}}=\left(j^{k} \varphi\right)^{*} i_{T\left(\Phi_{J^{k}} j^{k} B\right) j^{k} X} d\left(\Phi_{J^{k}} j^{k} B\right)^{*} \theta_{\mathfrak{L}}= \\
& =\left(j^{k} \varphi\right)^{*} i_{T\left(\Phi_{J^{k}} j^{k} B\right) j^{k} X} d \theta_{\mathfrak{L}}=0
\end{aligned}
$$

pois $j^{k} X$ vertical implica $T\left(\Phi_{J^{k}} j^{k} B\right) j^{k} X$ vertical, dado que

$$
\pi_{J^{k} E} \Phi_{J^{k}} j^{k} B=\tau_{J^{k}}\left\langle\sigma_{J^{k}}, \pi_{J^{k} E}\right\rangle_{1} j^{k} B=\tau_{J^{k}} j^{k} b \pi_{J^{k} E}=\tau b \pi_{J^{k} E}
$$

Assim, $\tilde{\varphi}$ é solução.

Por simplicidade, tomemos $r=1$. Agora, usaremos a proposição 2.2.5, com a notação descrita na observação que a segue.

Teorema 3.1.2. Denotemos por $\left(D_{\theta}, \mathcal{J}_{\theta}\right)$ o operador de Spencer, definido por $\theta_{\mathfrak{L}}$, sobre $\mathfrak{A}\left(J_{b} G\right)_{\pi_{A}} \times_{\pi_{J E}}$ JE. Se $G$ for grupoide de simetrias de $\theta_{\mathfrak{L}}$, então $D_{\theta}=0$.

Demonstração. Denotemos por $a_{J E}: \Gamma\left(\mathfrak{A}\left(J_{b} G\right)_{\pi_{A}} \times_{\pi_{J E}} J E\right) \rightarrow \mathfrak{X}(J E)$ a âncora do algebroide de ação e considere $\xi \in \Gamma\left(\mathfrak{A}\left(J_{b} G\right)_{\pi_{A}} \times_{\pi_{J E}} J E\right)$. Em primeiro lugar, por linearidade, podemos considerar $\xi$ na forma $1 \otimes \eta$, onde $\eta \in \mathfrak{A}\left(J_{b} G\right)$. Denotando o fluxo de $\eta$ por $\mathrm{Fl}^{\eta}$, temos que $a_{J E}$ é dada pela derivada, em cada $v \in J E$, de

$$
\Phi_{J}\left(\mathrm{Fl}_{t}^{\eta_{x}}, v\right)=\Phi_{J}\left(\mathrm{Fl}_{t}^{\eta} \pi_{J E}, i d_{J E}\right)(v)
$$

onde $x=\pi_{J E}(v)$. Como, para cada $t, \mathrm{Fl}_{t}^{\eta}$ é jato de bisseção de $G$, temos

$$
\left(\Phi_{J}\left(\mathrm{Fl}_{t}^{\eta} \pi_{J E}, i d_{J E}\right)\right)^{*} \theta_{\mathfrak{L}}=\theta_{\mathfrak{L}}
$$

Assim,

$$
D_{\theta}(\xi)=\mathcal{L}_{a_{J E}(\xi)} \theta_{\mathfrak{L}}=\left.\frac{d}{d t}\left(\left(\Phi_{J}\left(\mathrm{Fl}_{t}^{\eta} \pi_{J E}, i d_{J E}\right)\right)^{*} \theta_{\mathfrak{L}}\right)\right|_{t=0}=\left.\frac{d}{d t}\left(\theta_{\mathfrak{L}}\right)\right|_{t=0}=0
$$

que conclui a demonstração. 
É lícito então redigir a seguinte definição.

Definição 3.1.9. Uma corrente de Noether para o grupoide de simetrias acima é a imagem de um elemento $\xi \in \Gamma\left(\mathfrak{A}\left(J_{b} G\right) \pi_{\pi_{A}} \times_{\pi_{J E}} J E\right)$, segundo a aplicação $\mathcal{J}_{\theta}: \Gamma\left(\mathfrak{A}\left(J_{b} G\right) \pi_{\pi_{A}} \times_{\pi_{J E}} J E\right) \rightarrow$ $\Omega^{m-1}(J E)$, parte do operador de Spencer definido por $\theta_{\mathfrak{L}}$.

Observação 3.1.4. Em outras palavras,

$$
\mathcal{J}_{\theta}(\xi)=i_{a_{J E}(\xi)} \theta_{\mathfrak{L}}
$$

como era de se esperar. $\diamond$

Estamos aptos agora a enunciar o primeiro teorema de Noether.

Teorema 3.1.3. Em soluções das equações de Euler-Lagrange, a corrente de Noether é conservada.

Demonstração. Para uma solução $\varphi$ e um $\xi \in \Gamma\left(\mathfrak{A}\left(J_{b} G\right)_{\pi_{A}} \times_{\pi_{J E}} J E\right)$, o enunciado é expresso como

$$
d\left((j \varphi)^{*} \mathcal{J}_{\theta}(\xi)\right)=0
$$

Note então que

$$
d\left((j \varphi)^{*} \mathcal{J}_{\theta}(\xi)\right)=(j \varphi)^{*} d i_{a_{J E}(\xi)} \theta_{\mathfrak{L}}=(j \varphi)^{*} \mathcal{L}_{a_{J E}(\xi)} \theta_{\mathfrak{L}}-(j \varphi)^{*} i_{a_{J E}(\xi)} d \theta_{\mathfrak{L}}=-(j \varphi)^{*} i_{a_{J E}(\xi)} d \theta_{\mathfrak{L}}
$$

pois $a_{J E}(\xi)$ é "simetria infinitesimal" de $\theta_{\mathfrak{L}}$. Seja $\eta \in \Gamma\left(\mathfrak{A}\left(J_{b} G\right)\right)$, o elemento tal que

$$
\eta \pi_{J E}=\left\langle\pi_{A}, \pi_{J E}\right\rangle_{1} \xi
$$

Denotando a âncora de $\mathfrak{A}\left(J_{b} G\right)$ por $a_{M}: \mathfrak{A}\left(J_{b} G\right) \rightarrow T M$, a propriedade de ação $\pi_{J E} \Phi_{J}=\tau_{J}\left\langle\sigma_{J}, \pi_{J E}\right\rangle_{1}$, fornece $a_{J E}(\xi)$ projetável sobre $a_{M}(\eta)$. Se nos restringirmos à imagem de $j \varphi, a_{J E}(\xi)-T(j \varphi) a_{M}(\eta)$ é um campo vertical, uma vez que

$$
T \pi_{J E} a_{J E}(\xi)-T \pi_{J E} T(j \varphi) a_{M}(\eta)=a_{M}(\eta)-a_{M}(\eta)=0
$$

Assim,

$$
\begin{aligned}
(j \varphi)^{*} i_{a_{J E}(\xi)} d \theta_{\mathfrak{L}}=(j \varphi)^{*} i_{a_{J E}(\xi)-T(j \varphi) a_{M}(\eta)} d \theta_{\mathfrak{L}}+(j \varphi)^{*} i_{T(j \varphi) a_{M}(\eta)} d \theta_{\mathfrak{L}} & = \\
& =(j \varphi)^{*} i_{T(j \varphi) a_{M}(\eta)} d \theta_{\mathfrak{L}}
\end{aligned}
$$

pois $j \varphi$ é solução das equações de Euler-Lagrange. Como $T(j \varphi)$ é injetora, ainda restritos à imagem de $j \varphi$, podemos escrever

$$
(j \varphi)^{*} i_{T(j \varphi) a_{M}(\eta)} d \theta_{\mathfrak{L}}=i_{a_{M}(\eta)}(j \varphi)^{*} d \theta_{\mathfrak{L}}=0
$$

pois $(j \varphi)^{*} d \theta_{\mathfrak{L}}$ é uma $(m+1)$-forma diferencial sobre uma variedade diferenciável de dimensão $m$. Substituindo este resultado em 3.2 e esse em 3.1, ficamos com

$$
d\left((j \varphi)^{*} \mathcal{J}_{\theta}(\xi)\right)=0
$$

Também, temos o segundo teorema de Noether.

Teorema 3.1.4. Em soluções das equações de Euler-Lagrange, a corrente de Noether se anula.

Demonstração. Primeiro, note que, de sua definição, $\mathcal{J}_{\theta}$ é $C^{\infty}(M)$-linear. Então, dado 
$\xi \in \Gamma\left(\mathfrak{A}\left(J_{b} G\right)_{\pi_{A}} \times_{\pi_{J E}} J E\right)$, para toda $f \in C^{\infty}(M)$ e toda solução $\varphi$,

$$
\begin{aligned}
& d\left((j \varphi)^{*} \mathcal{J}_{\theta}(f \xi)\right)=0 \\
& d\left((j \varphi)^{*} f \cdot \mathcal{J}_{\theta}(\xi)\right)=0 \\
& (j \varphi)^{*} d f \wedge(j \varphi)^{*} \mathcal{J}_{\theta}(\xi)+(j \varphi)^{*} f \cdot(j \varphi)^{*} d \mathcal{J}_{\theta}(\xi)=0 \\
& (j \varphi)^{*} d f \wedge(j \varphi)^{*} \mathcal{J}_{\theta}(\xi)=0
\end{aligned}
$$

Como $J E$ admite cartas fibradas, $j \varphi$ é seção (holônoma) de $\pi_{J E}$ e $f$ é arbitrária, só pode ser

$$
(j \varphi)^{*} \mathcal{J}_{\theta}(\xi)=0
$$

que mostra o resultado.

Observação 3.1.5. A separação dos dois resultados anteriores é conceitualmente útil. Mais adiante, ao discutirmos brevemente resultados análogos, referentes a simetrias em um sentido mais amplo, veremos que o primeiro teorema de Noether persiste, sendo porém ligeiramente modificado, enquanto que o segundo, deixa de valer. Para uma discussão sobre o significado dos teoremas de Noether aqui expostos, bem como outras questões sobre correntes e o tensor energia-momento, queira consultar [12]. Na mesma referência, encontra-se uma discussão que aponta que o conceito de corrente de Noether elaborado aqui é o de corrente total "melhorada" (improved). Assim, aqui não há necessidade de tal processo (o processo de improvement), nem de termos de correção. $\diamond$

Observação 3.1.6. O leitor atento deve ter notado que não fizemos muita distinção entre os conceitos de corrente de Noether como forma diferencial em $J E$ e como forma diferencial em $M$, dada pelo pull-back por um prolongamento de seção $\varphi$. Apenas para esclarecer, na literatura, às vezes estes conceitos são referidos como "corrente independente de campos" e "corrente dependente do campo", respectivamente. Visto que sempre óbvia, não faremos mais referências à distinção. $\diamond$

Observação 3.1.7. Optamos por não discutir o conceito de "tensor energia-momento". Tal discussão envolveria os detalhes sobre a cisão da derivada variacional da lagrangiana em um operador de Euler-Lagrange e um operador de Poincaré-Cartan, como mencionado na observação 3.1.3. Grosso modo, uma vez escolhida uma conexão linear e simétrica em $M$, obtemos uma escolha canônica de $\theta_{\mathfrak{L}}$ e um levantamento horizontal (induzido) que permite dizer quando uma transformação é puramente horizontal (i.e., sem variação nas fibras). O tensor energia-momento é então construído a partir destes dados. Para mais informações, o leitor pode consultar $[10 ; 25]$. $\diamond$

\subsection{Teorias de calibre}

O primeiro teorema de Noether, na seção anterior, estabelece uma relação entre quantidades conservadas e simetrias. Agora, em física, quantidades não são conservadas apenas globalmente, mas localmente também. Se pensarmos em $M$ como o espaço-tempo de Minkowski, as simetrias esperadas são as dadas pelas transformações de Lorentz, translações espaço-temporais e rotações, que fornecem a conservação de energia, momento linear e momento angular. Para a conservação de carga elétrica, por exemplo, ser expressa por uma simetria, graus de liberdade adicionais devem ser considerados, bem como simetrias adicionais. Isto é realizado através da adição de um espaço "interno" ao espaço-tempo, no qual age um grupo de simetrias. Em outras palavras, esta é uma teoria de campos na qual o espaço de configurações é, ao menos em princípio, um fibrado associado a um fibrado principal, onde a base é o espaço-tempo e o grupo estrutural é o grupo de simetrias internas. Assim, as simetrias internas são dadas por automorfismos estritos do fibrado associado, enquanto que simetrias gerais, constituídas de internas e de simetrias na base, são dadas por automorfismos gerais. É neste ponto que a teoria de grupoides de Lie expressa todo o seu potencial.

Será suficiente, para nossos propósitos, considerar teorias nas quais a lagrangiana é de primeira ordem. O leitor não deverá ter dificuldades em adaptar as técnicas aqui desenvolvidas a casos de ordem superior. 
Fixemos então um fibrado principal $(P, M, \rho, G), \operatorname{com} \operatorname{dim}(M)=m$ e uma variedade suave $Q$, sobre a qual $G$ age à esquerda, tornando possível a construção do fibrado associado $\left(P \times{ }_{G} Q, M, \pi_{Q}\right)$. A primeira "aposta" é considerar uma lagrangiana $\mathfrak{L} \in \Omega^{m}\left(J\left(P \times_{G} Q\right)\right)$ (lembrando que $\mathfrak{L}$ é horizontal). Na literatura (confira [13]) é comum uma escolha tácita de uma conexão principal, que permite que identifiquemos $J\left(P \times_{G} Q\right)$ e $\vec{J}\left(P \times_{G} Q\right)$ (veja [25]). Usando a projeção $D$, desenvolvida na proposição 2.3.2, podemos construir $D^{*} \mathfrak{L} \in \Omega^{m}\left(C P{ }_{\pi_{C P}} \times_{\pi_{J P Q}} J\left(P \times_{G} Q\right)\right)$, que fornece uma lagrangiana que depende explicitamente de conexões principais. Quanto à invariância destes objetos pela ação do grupoide de calibre $P \times_{G} P$, apesar de evidente a necessidade de invariância por automorfismos estritos, o que admitiremos de agora em diante, não se espera (e é até mesmo indesejável) que sejam invariantes por todos os automorfismos da estrutura. Projetado, este seria um grupo de difeomorfismos na base grande demais (mas, em geral, isto não corresponde a todos os difeomorfismos na base, como mostrado em [3]). Ao grupo de bisseções para as quais a lagrangiana é invariante (qualquer uma delas), damos o nome de grupo de estabilidade da lagrangiana. Caso defina um subgrupoide do grupoide envolvido, tal grupoide será dito grupoide de estabilidade da lagrangiana (confira a observação 2.3.2). Entretanto, é necessária alguma discussão sobre este conceito de invariância.

Observação 3.2.1. Nota sobre o conceito de invariância de uma lagrangiana.

Do modo como foi enunciado, pode parecer que a invariância de uma lagrangiana por determinadas bisseções seja o conceito adequado para lidarmos. O ponto é que, se a lagrangiana for invariante por uma dada bisseção, o funcional ação também o será. Porém, se uma dada bisseção modificar a lagrangiana por uma forma de contato ou por um diferencial exato, a lagrangiana não será invariante no sentido aqui adotado, mas o funcional ação será. Mais correto seria modificar o conceito de bisseções que deixam $\theta_{\mathfrak{L}}$ invariante, tendo em mente todas as ambiguidades que isto ocasionaria. Discutiremos isto na seção 3.3. Agora, no que segue, o leitor poderá notar que tudo o que será feito valeria para uma outra forma diferencial; não é usado o fato de uma lagrangiana ser horizontal. Ainda, como mencionado na seção anterior, mediante uma escolha de uma conexão adequada, podemos relacionar soluções dadas em termos de $(j \varphi)^{*} \mathfrak{L}$ a soluções dadas em termos de $(j \varphi)^{*} \theta_{\mathfrak{L}}$, para uma $\theta_{\mathfrak{L}}$ específica, dado que $\theta_{\mathfrak{L}}$ e $\mathfrak{L}$ diferem por termos de contato, ou seja, $(j \varphi)^{*} \theta_{\mathfrak{L}}=(j \varphi)^{*} \mathfrak{L}$, qualquer que seja $j \varphi$. Portanto, procederemos com a análise do grupo de estabilidade de $\mathfrak{L}$, como se este fosse o essencial, por simplicidade de notação. Mais próximo do correto seria analisar o grupo de estabilidade de $\theta_{\mathfrak{L}}$, com uma definição mais ampla de estabilidade, que contém (possivelmente propriamente) o anterior. Da natureza das técnicas empregadas, não há prejuízos ao resultado, desde que tenhamos em mente que este grupo de bisseções pode ser menor do que o grupo que mantém o funcional ação invariante. De qualquer modo, esta simplificação cobre a maioria dos casos de interesse em teorias de calibre ${ }^{2} . \diamond$

Levada em conta a observação anterior, relacionemos os grupos de estabilidade de $\mathfrak{L}$ e $D^{*} \mathfrak{L}$.

Teorema 3.2.1 (Acoplamento mínimo). Existe uma correspondência biunívoca entre lagrangianas $\overrightarrow{\mathfrak{L}} \in \Omega^{m}\left(\vec{J}\left(P \times_{G} Q\right)\right)$ e lagrangianas $\mathfrak{L} \in \Omega^{m}\left(C P{ }_{\pi_{C P}} \times_{\pi_{J P Q}} J\left(P \times_{G} Q\right)\right)$, que tenham grupos de estabilidade compativeis.

Demonstração. Ao tomarmos uma lagrangiana $\overrightarrow{\mathfrak{L}} \in \Omega^{m}\left(\vec{J}\left(P \times{ }_{G} Q\right)\right)$, podemos construir $\mathfrak{L}=D^{*} \overrightarrow{\mathfrak{L}} \in$ $\Omega^{m}\left(C P \pi_{C P} \times_{\pi_{J C P}} J\left(P \times_{G} Q\right)\right)$, que terá seu grupo de estabilidade definido pelo teorema 2.3.2.

Por outro lado, ao tomarmos uma lagrangiana $\mathfrak{L} \in \Omega^{m}\left(C P \pi_{C P} \times_{\pi_{J C P}} J\left(P \times_{G} Q\right)\right)$, podemos definir uma lagrangiana $\overrightarrow{\mathfrak{L}} \in \vec{J}\left(P \times_{G} Q\right)$, em elementos, por

$$
\overrightarrow{\mathfrak{L}}\left(\vec{j}_{x} \psi_{Q}\right)=\mathfrak{L}\left(A_{x}, j_{x} \varphi_{Q}\right)
$$

onde $j_{x} \psi_{Q}=D\left(A_{x}, j_{x} \varphi_{Q}\right)$. Isto é possível pelo seguinte fato. Novamente, raciocinemos em termos de germes de seções. Se $\left(s, \rho_{Q}(\varphi, f)\right)$ e $\left(\tilde{s}, \rho_{Q}(\tilde{\varphi}, \tilde{f})\right)$ forem tais que $D_{x}\left(s, \rho_{Q}(\varphi, f)\right)=D_{x}\left(\tilde{s}, \rho_{Q}(\tilde{\varphi}, \tilde{f})\right)$, então

$$
\rho_{Q}(\tilde{s}(x), l(\delta(\tilde{s}, \tilde{\varphi}), \tilde{f}))=\rho_{Q}(s(x), l(\delta(s, \varphi), f))
$$

\footnotetext{
${ }^{2} \mathrm{O}$ leitor pode encontrar uma discussão sobre isso em [16].
} 
Por simplicidade, podemos tomar $\tilde{s}(x)=s(x)$. Com isto, a condição de verticalidade fornece

$$
l(\delta(\tilde{s}, \tilde{\varphi}), \tilde{f})=l(\delta(s, \varphi), f)
$$

o que leva a

$$
\tilde{f}=l(m(\delta(\tilde{\varphi}, \tilde{s}), \delta(s, \varphi)), f)
$$

Agora, tomemos a aplicação $\rho_{P}(\tilde{s}, s)$. É claramente uma bisseção, que corresponde a um automorfismo (local) estrito de $P$. Temos que $\rho_{P}(\tilde{s}, s)$ leva $\left(s, \rho_{Q}(\varphi, f)\right)$ em $\left(\tilde{s}, \rho_{Q}(\tilde{\varphi}, \tilde{f})\right)$. Para ver isto, basta fazer

$$
\begin{aligned}
& \Phi_{\mathcal{G} C P Q}\left(\rho_{P}\left(\tilde{s}, \rho_{Q}(\varphi, f)\right)\right)=\left(r(\tilde{s}, \delta(s, s)), \rho_{Q}(r(\tilde{s}, \delta(s, \varphi)), f)\right)= \\
& =\left(\tilde{s}, \rho_{Q}(\tilde{s}, l(\delta(s, \varphi), f))\right)=\left(\tilde{s}, \rho_{Q}(r(\tilde{s}, \delta(\tilde{s}, \tilde{\varphi})), l(\delta(\tilde{\varphi}, \tilde{s}), l(\delta(s, \varphi), f)))\right)= \\
& \quad=\left(\tilde{s}, \rho_{Q}(\tilde{\varphi}, l(m(\delta(\tilde{\varphi}, \tilde{s}), \delta(s, \varphi)), f))\right)=\left(\tilde{s}, \rho_{Q}(\tilde{\varphi}, \tilde{f})\right)
\end{aligned}
$$

Deste modo, podemos construir uma bisseção $B$ que leva $\left(A_{x}, j_{x} \varphi_{Q}\right)$ em $\left(\tilde{A}_{x}, j_{x} \tilde{\varphi}_{Q}\right)$ e que é $\mathfrak{L}$ holônoma, pois corresponde a automorfismo estrito de $P$. Portanto,

$$
\left(\tilde{A}_{x}, j_{x} \tilde{\varphi}_{Q}\right)^{*} \mathfrak{L}=\left(\Phi_{J C P Q} B\left(A_{x}, j_{x} \varphi_{Q}\right)\right)^{*} \mathfrak{L}=\left(A_{x}, j_{x} \varphi_{Q}\right)^{*}\left(\Phi_{J C P Q} B\right)^{*} \mathfrak{L}=\left(A_{x}, j_{x} \varphi_{Q}\right)^{*} \mathfrak{L}
$$

Assim, $\overrightarrow{\mathfrak{L}}$ é bem definida e ainda, $\mathfrak{L}=D^{*} \overrightarrow{\mathfrak{L}}$. Finalmente, para toda bisseção $B, \mathfrak{L}$-holônoma, temos uma bisseção $\bar{B}$, tal que o par $(B, \bar{B})$ seja compatível, e temos

$$
\begin{aligned}
& (\vec{j} \psi)^{*}\left(\Phi_{\lambda} \bar{B}\right)^{*} \overrightarrow{\mathfrak{L}}=(A, j \varphi)^{*} D^{*}\left(\Phi_{\lambda} \bar{B}\right)^{*} \overrightarrow{\mathfrak{L}}=(A, j \varphi)^{*}\left(\Phi_{\lambda} \bar{B} D\right)^{*} \overrightarrow{\mathfrak{L}}= \\
& =(A, j \varphi)^{*}\left(\Phi_{\lambda}(\lambda, D) B\right)^{*} \overrightarrow{\mathfrak{L}}=(A, j \varphi)^{*}\left(D \Phi_{J C P Q} B\right)^{*} \overrightarrow{\mathfrak{L}}=(A, j \varphi)^{*}\left(\Phi_{J C P Q} B\right)^{*} D^{*} \overrightarrow{\mathfrak{L}}= \\
& =(A, j \varphi)^{*}\left(\Phi_{J C P Q} B\right)^{*} \mathfrak{L}=(A, j \varphi)^{*} \mathfrak{L}=(A, j \varphi)^{*} D^{*} \overrightarrow{\mathfrak{L}}=(\vec{j} \psi)^{*} \overrightarrow{\mathfrak{L}}
\end{aligned}
$$

Da arbitrariedade de $\vec{j} \psi$, ficamos com

$$
\left(\Phi_{\lambda} \bar{B}\right)^{*} \overrightarrow{\mathfrak{L}}=\overrightarrow{\mathfrak{L}}
$$

mostrando que $\bar{B}$ é $\mathfrak{L}$-holônoma. Portanto, segue a bijeção entre os conjuntos de lagrangianas enunciados, de maneira que seus grupos de estabilidade sejam compatíveis.

Recapitulemos o significado disto, do ponto de vista físico. Começamos "postulando" uma teoria que admitia simetrias internas (ao espaço-tempo), o que levava a uma lagrangiana em $\vec{J}\left(P \times{ }_{G} Q\right)$, para algum fibrado associado $P \times_{G} Q$, que dependia da escolha de uma conexão. As simetrias internas, neste caso, ou não eram covariantes (quebrando a invariância da lagrangiana), ou eram tomadas como a mesma em todos os pontos, correspondendo portanto, a simetrias globais. Para remediar este problema, introduzimos lagrangianas que dependam explicitamente de conexões principais, o que levou a uma lagrangiana em $C P{ }_{\pi_{C P}} \times_{\pi_{J P Q}} J\left(P \times_{G} Q\right)$. Para ser útil, tal construção deveria ser canônica e a lagrangiana resultante deveria exibir, não apenas a mesma invariância com relação a transformações nas fibras, mas a mesma invariância com relação a transformações na base. Esta é a necessidade e o conteúdo do teorema anterior. Porém, se parássemos aí, teríamos uma teoria incompleta, pois introduzimos quantidades adicionais, as conexões principais, que agora são campos da teoria, devendo portanto, obedecer a uma dinâmica. Logo, a lagrangiana total deve conter informação sobre derivadas de conexões. Isto corresponde à ideia de tomarmos $C P{ }_{\pi_{C P}} \times_{\pi_{Q}}\left(P \times_{G} Q\right)$ como fibrado de configurações e a lagrangiana em $J\left(C P{ }_{\pi_{C P}} \times_{\pi_{Q}}\left(P \times_{G} Q\right)\right)$, com o adicional de que temos uma separação clara entre um setor puramente de calibre, aqui denotado por $\mathfrak{L}_{G a u} \in \Omega^{m}(J(C P))$ e um setor de "matéria", denotado doravante por $\mathfrak{L}_{\text {mat }} \in \Omega^{m}\left(C P{\pi_{C P}}_{\pi_{J P Q}} J\left(P \times_{G} Q\right)\right)$. O novo setor de calibre deve, portanto, exibir uma invariância por transformações de maneira independente do setor de matéria, que já exibe sua própria. A interpretação geométrica disto é que este setor fornece a informação de como as conexões variam de ponto a ponto, ou seja, fornece a interação 
proposta, modelada como uma curvatura em um espaço adequado. No caso do eletro-magnetismo, por exemplo, este é o setor que informa sobre o campo eletro-magnético em si. Assim, para ser um conceito matematicamente representativo para a física, as lagrangianas definidas em $J(C P)$ devem ser "naturais" com relação ao operador curvatura. Este é o conteúdo do próximo teorema.

Teorema 3.2.2 (Utiyama). Existe uma correspondência biunívoca entre lagrangianas $\mathfrak{L}_{\text {curv }} \in$ $\Omega^{m}\left(\left(P \times_{G} \mathfrak{g}\right) \otimes \bigwedge^{2} T^{*} M\right)$ e lagrangianas $\mathfrak{L}_{\text {Gau }} \in \Omega^{m}(J(C P))$, que tenham grupos de estabilidade compativeis.

Demonstração. Apresentaremos apenas um esboço de demonstração, por ser muito semelhante à do teorema 3.2.1. Tomemos $\mathfrak{L}_{\text {curv }} \in \Omega^{m}\left(\left(P \times_{G} \mathfrak{g}\right) \otimes \bigwedge^{2} T^{*} M\right)$. Definindo $\mathfrak{L}_{\text {Gau }}=R^{*} \mathfrak{L}_{\text {curv }}$, temos que ambas terão grupos de estabilidade compatíveis, devido ao que foi dito na observação 2.3.4, tendo em vista o teorema 2.3.3.

Por outro lado, tomando $\mathfrak{L}_{G a u} \in \Omega^{m}(J(C P))$, defina $\mathfrak{L}_{\text {curv }}$, em elementos, por

$$
\mathfrak{L}_{\text {curv }}(\xi \otimes \omega)=\mathfrak{L}_{\text {Gau }}(\gamma)
$$

para $R_{\gamma}=\xi \otimes \omega$. Que $\mathfrak{L}_{\text {curv }}$ é bem definida, segue da invariância de $\mathfrak{L}_{\text {Gau }}$ por automorfismos estritos. Também, um cálculo análogo ao efetuado na demonstração do teorema anterior, mostra que, dada uma bisseção $\mathfrak{L}_{G a u}$-holônoma, ela se "projeta" a uma bisseção (compatível) $\bar{B}$, que será $\mathfrak{L}_{\text {curv }}$-holônoma, fornecendo o resultado.

Deste modo, lagrangianas no setor de calibre se fatoram naturalmente a lagrangianas em $\left(P \times_{G} \mathfrak{g}\right) \otimes \bigwedge^{2} T^{*} M$, que são globalmente invariantes, pelo operador de curvatura.

De posse destas técnicas, é possível repetir o que foi feito na seção anterior e definir correntes de Noether. Vale notar que é necessário considerar a lagrangiana total, neste caso. É preciso notar que é a ação de $J_{b} J_{b}\left(P \times{ }_{G} Q\right)$ em $J(C P) \pi_{J C P} \times_{\pi_{J J P Q}} J J\left(P \times_{G} Q\right)$ que deve ser considerada, com uma simplificação dada pela condição de separação entre os setores de calibre e de matéria, possível, já que a projeção $\pi_{0, J P P^{-1}}^{1}: J_{b} J_{b}\left(P \times_{G} P\right) \rightarrow J_{b}\left(P \times_{G} P\right)$ é morfismo de grupoides de Lie e que uma ação em $J J E$, para um fibrado $E$ qualquer, induz uma ação em $J E$, por projeção. Apenas como uma pequena amostra desta necessidade, cito o caso do eletro-magnetismo. Lá, a variação da lagrangiana total, com relação às conexões principais, fornece as equações de Maxwell inomogêneas. Apenas a variação do setor de calibre, com relação às conexões principais, fornece as equações de Maxwell homogêneas, tão somente (o que é de se esperar, uma vez que não há matéria).

Assim, com esta espécie de exemplo de brinquedo, esperamos ter motivado o leitor a encarar as técnicas aqui descritas como parte de um conjunto consistente de ferramentas que se ajustam bem à solução dos tipos de problemas que surgem ao lidarmos com teorias de campos.

\subsection{Simetrias em um sentido mais amplo}

Voltando à situação geral da seção 3.1, fixemos uma forma de Poincaré-Cartan de primeira ordem (por simplicidade) $\theta_{\mathfrak{L}} \in \Omega^{m}(J E)$, para a lagrangiana $\mathfrak{L}$. Se um automorfismo $\left(\phi_{E}, \phi_{M}\right)$ de $E$ modificar $\theta_{\mathfrak{L}}$ por uma forma de contato ou por uma forma diferencial exata, o funcional ação permanecerá o mesmo. É claro que isto não se encaixa no conceito de simetria para $\theta_{\mathfrak{L}}$, elaborada na definição 3.1.8. Agora, devido ao teorema de Noether, não considerar tais simetrias poderia levar a ignorarmos leis de conservação. Daí a necessidade desta correção.

Definição 3.3.1. Seja $\Phi: G_{\sigma} \times_{\pi} E \rightarrow E$ uma ação suave do grupoide de Lie $(G, M, \sigma, \tau, \mu, \varepsilon, \iota)$, no fibrado suave $(E, M, \pi)$. Dizemos que uma bisseção verticalmente constante $B \in \operatorname{Bis}\left(G_{\sigma} \times_{\pi} E\right)$ é uma simetria ampla para $\theta_{\mathfrak{L}}$, quando

$$
(j B)^{*} \theta_{\mathfrak{L} \Phi_{J}}=\alpha+d f
$$


onde $\alpha$ é $m$-forma de contato, $f$ é $(m-1)$-forma arbitrária, $\theta_{\mathfrak{L} \Phi_{J}}$ é (como de costume) dada por

$$
\theta_{\mathfrak{L} \Phi_{J}}=\left\langle\sigma_{J}, \pi_{J E}\right\rangle_{2}^{*} \theta_{\mathfrak{L}}-\Phi_{J}^{*} \theta_{\mathfrak{L}}
$$

$e \Phi_{J}: J_{b} G_{\sigma_{J}} \times_{\pi_{J E}} J E \rightarrow J E$ denota o prolongamento de $\Phi$.

Definição 3.3.2. Um grupoide de simetrias amplas para $\theta_{\mathfrak{L}}$ é um grupoide de Lie $(G, M, \sigma, \tau, \mu, \varepsilon, \iota)$, tal que exista $\Phi: G_{\sigma} \times_{\pi} E \rightarrow E$, de modo que toda bisseção verticalmente constante do grupoide de ação de $\Phi$ seja simetria ampla.

Obviamente, isto não seria útil se não fosse válida a seguinte proposição.

Proposição 3.3.1. Simetrias amplas levam soluções em soluções.

Demonstração. Tomemos uma simetria ampla $B \in \operatorname{Bis}\left(G_{\sigma} \times_{\pi} E\right)$ de modo que tenhamos

$$
\left(\Phi_{J} j B\right)^{*} \theta_{\mathfrak{L}}=\theta_{\mathfrak{L}}+\alpha+d f
$$

$\operatorname{com} \alpha$ forma de contato. Para $\varphi \in \Gamma_{l o c}(E)$, temos que $B$ leva $\varphi$ em

$$
\tilde{\varphi}=\Phi B \varphi(\tau b)^{-1}
$$

onde $b$ é dada por $B=\left(b \pi, i d_{E}\right)$. Assim,

$$
j \tilde{\varphi}=\Phi_{J} j B j \varphi
$$

Se $\varphi$ for solução das equações de Euler-Lagrange, teremos

$$
\begin{aligned}
(j \tilde{\varphi})^{*} i_{j X} d \theta_{\mathfrak{L}}=\left(\Phi_{J} j B j \varphi\right)^{*} i_{j X} d \theta_{\mathfrak{L}}=(j \varphi)^{*} i_{T\left(\Phi_{J} j B\right) j X} d\left(\Phi_{J} j B\right)^{*} \theta_{\mathfrak{L}} & = \\
=(j \varphi)^{*} i_{T\left(\Phi_{J} j B\right) j X} d \theta_{\mathfrak{L}}+(j \varphi)^{*} i_{T\left(\Phi_{J} j B\right) j X} d \alpha & =i_{T\left(j \varphi \Phi_{J} j B\right) j X}(j \varphi)^{*} d \alpha=0
\end{aligned}
$$

para toda deformação $X$, onde usamos que $\varphi$ é solução, que $T\left(j \varphi \Phi_{J} j B\right) j X$ é vertical, que $\alpha$ é forma de contato e que $d d f=0$. Logo $\tilde{\varphi}$ é solução.

Proposição 3.3.2. Seja $\Phi: G_{\sigma} \times_{\pi} E \rightarrow E$ uma ação suave do grupoide de Lie $(G, M, \sigma, \tau, \mu, \varepsilon, \iota)$, no fibrado suave $(E, M, \pi)$. O conjunto das simetrias amplas de $\theta_{\mathfrak{L}}$ é subgrupo do grupo de bisseções verticalmente constantes do grupoide de ação de $\Phi$.

Demonstração. A bisseção unidade é claramente simetria ampla. Tomemos então $B, \tilde{B}$ simetrias amplas de $\theta_{\mathfrak{L}}$, de modo que

$$
\begin{aligned}
& (j B)^{*} \theta_{\mathfrak{L} \Phi_{J}}=\alpha+d f \\
& (j \tilde{B})^{*} \theta_{\mathfrak{L} \Phi_{J}}=\tilde{\alpha}+d \tilde{f}
\end{aligned}
$$

$\operatorname{com} \alpha, \tilde{\alpha}$ formas de contato. Então,

$$
\begin{aligned}
& \left(m_{\Phi_{J}}(j \tilde{B}, j B)\right)^{*} \theta_{\mathfrak{L} \Phi_{J}}=\left(\mu_{\Phi_{J}}\left(j \tilde{B} \Phi_{J} j B, j B\right)\right)^{*} \theta_{\mathfrak{L} \Phi_{J}}=\left(j \tilde{B} \Phi_{J} j B, j B\right)^{*} \mu_{\Phi_{J}}^{*} \theta_{\mathfrak{L} \Phi_{J}}= \\
& =\left(j \tilde{B} \Phi_{J} j B, j B\right)^{*}\left\langle\sigma_{\Phi_{J}}, \tau_{\Phi_{J}}\right\rangle_{1}^{*} \theta_{\mathfrak{L} \Phi_{J}}+\left(j \tilde{B} \Phi_{J} j B, j B\right)^{*}\left\langle\sigma_{\Phi_{J}}, \tau_{\Phi_{J}}\right\rangle_{2}^{*} \theta_{\mathfrak{L} \Phi_{J}}= \\
& =\left(j \tilde{B} \Phi_{J} j B\right)^{*} \theta_{\mathfrak{L} \Phi_{J}}+(j B)^{*} \theta_{\mathfrak{L} \Phi_{J}}=\left(\Phi_{J} j B\right)^{*} \tilde{\alpha}+\left(\Phi_{J} j B\right)^{*} d \tilde{f}+\alpha+d f= \\
& =\left(\Phi_{J} j B\right)^{*} \tilde{\alpha}+\alpha+d\left(f+\left(\Phi_{J} j B\right)^{*} \tilde{f}\right)
\end{aligned}
$$

onde usamos que $\theta_{\mathfrak{L} \Phi_{J}}$ é forma multiplicativa. A proposição estará demonstrada ao provarmos que $\left(\Phi_{J} j B\right)^{*} \tilde{\alpha}$ é forma de contato. Agora, como $\left(\Phi_{J} j B\right)$ é prolongamento de $\Phi B$, que por sua vez é automorfismo que se projeta sobre um difeomorfismo na base (veja [37]), preserva o ideal de contato, donde segue o resultado.

O teorema 3.1.2, sobre simetrias infinitesimais, é modificado então para compensar os novos termos presentes. 
Teorema 3.3.1. Denotemos por $\left(D_{\theta}, \mathcal{J}_{\theta}\right)$ o operador de Spencer, definido por $\theta_{\mathfrak{L}}$, sobre $\mathfrak{A}\left(J_{b} G\right)_{\pi_{A}} \times_{\pi_{J E}} J E$. Se $G$ for grupoide de simetrias amplas para $\theta_{\mathfrak{L}}$, então $D_{\theta}=\alpha^{\prime}+d f^{\prime}$, onde $\alpha^{\prime}$ e $f^{\prime}$ denotam derivadas de curvas em formas de contato e arbitrárias, respectivamente.

Demonstração. Daremos uma ideia da demonstração. Como feito no teorema 3.1.2, substituímos o resultado do fluxo da ação por

$$
\left(\Phi_{J}\left(\mathrm{Fl}_{t}^{\eta} \pi_{J E}, i d_{J E}\right)\right)^{*} \theta_{\mathfrak{L}}=\theta_{\mathfrak{L}}+\alpha_{t}+d f_{t}
$$

onde, para cada $t, \alpha_{t}$ é forma de contato e $d f_{t}$ é diferencial de forma arbitrária. Portanto,

$$
\mathcal{L}_{a(\eta)} \theta_{\mathfrak{L}}=\left.\frac{d \alpha_{t}}{d t}\right|_{t=0}+d\left(\left.\frac{d f_{t}}{d t}\right|_{t=0}\right)
$$

Assim, não há alteração no conceito de corrente de Noether, mas o primeiro teorema de Noether deve ser modificado para incluir estes termos adicionais. Neste caso, o teorema 3.1.3, o termo de contato não contribui, mas a diferencial total, sim. Repetindo os cálculos lá efetuados, vemos que

$$
d\left((j \varphi)^{*} \mathcal{J}_{\theta}(\xi)\right)=(j \varphi)^{*} d f^{\prime}
$$

Como consequência, a corrente de Noether é, por vezes, expressa como

$$
\mathcal{J}_{\theta}(\xi)-f^{\prime}
$$

onde $f^{\prime}$ deve ser determinada (ou definida) sem o uso das equações de movimento. Já o segundo teorema de Noether, o teorema 3.1.4, deixa de valer.

Isso mostra que o tratamento de simetrias amplas é consideravelmente mais complicado, se comparado ao de simetrias no sentido mais estrito, elaborado no início deste capítulo. 


\section{Epílogo}

O formalismo de ações de grupoides de Lie para descrever simetrias, desenvolvido aqui, é altamente "engessado". Foi exposto desta forma por melhor atender às necessidades do exemplo principal a ser coberto neste trabalho - a teoria clássica de campos. Entretanto, para um sistema de equações diferenciais parciais, descrito sobre um fibrado $(E, M, \pi)$, sobre o qual age um grupoide de Lie $G$, há inúmeras possibilidades de descrevermos simetrias para estas equações, não elaboradas aqui. Por exemplo, é possível que existam bisseções de $J_{b}^{r} G_{\sigma_{J^{r}}} \times_{\pi_{J^{r}}} J^{r} E$ que preservem a estrutura de contato, sendo portanto simetrias do sistema de equações (veja [27]), sem que sejam prolongamentos de bisseções verticalmente constantes de $G_{\sigma} \times_{\pi} E$. Há não só a questão da holonomia destas bisseções, mas também o fato de podermos ter bisseções verticais que não sejam verticalmente constantes. Poderíamos usar este tipo de bisseção para, por exemplo, construir uma simetria que seja uma parametrização de automorfismos do fibrado, ao longo das fibras. Como estruturas geométricas podem ser determinadas ao especificarmos um pseudogrupo de transformações [15; 20], ao menos em princípio, isto possibilitaria modelar uma família de estruturas geométricas em $E$, parametrizadas de maneira que seções diferentes de $E$ "percebam" uma geometria diferente. Explorar as inúmeras possibilidades abertas nesta direção pode ser terreno fértil para pesquisas futuras.

Algo interessante de ser notado é que o algebroide de Lie do grupoide de ação dá conta exatamente dos dados infinitesimais das simetrias, como expostas aqui, neste sentido mais limitado. Em outras palavras, ao ampliar o conceito de simetria para bisseções que não sejam prolongamentos de bisseções verticalmente constantes, o algebroide de Lie é insuficiente para descrever todos os dados infinitesimais.

Um ponto a favor da descrição de simetrias infinitesimais em termos de um algebroide de Lie é que, uma vez escolhido um "dual" para tal algebroide (visto como fibrado vetorial), a álgebra de Gerstenhaber induzida na álgebra exterior de seu espaço de seções por seu colchete de Lie, dá origem a um diferencial exterior no espaço de seções deste dual, donde obtemos uma álgebra diferencial graduada [8]. Agora, na presença de simetrias, não podemos ter esperanças de que a transformada de Legendre da lagrangiana seja regular. Porém, em condições suficientemente boas, podemos considerar um fibrado vetorial $V$ que ofereça uma resolução para o "ideal" associado a esta degenerescência. Assim, teríamos um complexo do tipo Koszul-Tate, equivariante, que aliado à álgebra diferencial graduada anterior fornece uma construção do tipo BRST, semelhante à técnica empregada em [11]. Tal construção melhora o entendimento da relação entre as prescrições lagrangiana e hamiltoniana da teoria clássica de campos, bem como a relação entre simetrias e vínculos, presente no lado hamiltoniano. Digno de nota, é que isto é válido mesmo fora do ambiente de teorias de calibre, ou seja, sem a necessidade das simetrias estarem associadas à noção de fibrado principal. Tudo o que é necessário é que as simetrias sejam descritas por um grupoide de Lie (de maneira suficientemente regular).

Outra vantagem da abordagem de simetrias em termos de ações de grupoides de Lie é sua rica estrutura algébrica. Ao considerarmos as álgebras de funções suaves das variedades suaves envolvidas, podemos ver que a estrutura de grupoide de Lie dá origem a um algebroide de Hopf, ou seja, um cogrupoide nesta categoria de álgebras. Ainda, a ação em si fornece um algebroide de Hopf de ação. Isto pode levar a uma outra abordagem de resoluções e vínculos, mencionada acima. A relação destes objetos algébricos com as álgebras de Lie-Rinehart oriundas diretamente da construção dos algebroides de Lie, pode ser bastante profícua.

A questão de considerarmos ou não grupoides de Lie em geral, e não apenas os advindos de 
fibrados principais, é delicada. Em princípio, quando consideramos teorias de campos que modelam aspectos fundamentais do universo, se as simetrias da teoria puderem ser modeladas por um grupoide de Lie, então a hipótese de que as leis da física obedecem critérios de isotropia e homogeneidade fornece um grupoide de Lie transitivo, que portanto, é grupoide de calibre de algum fibrado principal. Disto, vemos que toda teoria física suficientemente geral sobre simetrias, deveria poder ser expressa como uma teoria de calibre (ou algo muito próximo a isto). Porém, em certas aplicações, pode ser desejável considerar descontinuidades na base, onde temos propriedades diferentes em diferentes regiões. É o que acontece, por exemplo, ao modelarmos meios dielétricos com permissividades diferentes, ou mesmo cristais que possuam alguma anisotropia óptica. Tais descontinuidades são admissíveis, do ponto de vista clássico. Um grupoide de Lie geral é capaz de particionar a base em setores de modo que, em cada setor, forneça um grupoide de calibre diferente.

Há muito ainda a ser explorado. 


\section{Referências Bibliográficas}

[1] Abad, C.A., Crainic, M.: Representations up to homotopy of Lie algebroids, arXiv:0901.0319v2 [math.DG]. 32

[2] Abad, C.A., Crainic, M.: The Weil algebra and the van Est isomorphism, arXiv:0901.0322 [math.DG]. 30

[3] Abbati, M.C., Cirelli, R., Mania', A., Michor, P.: The Lie group of automorphisms of a principal bundle, J. Geom. Phys. 6 (1989) 215-235. 52

[4] Adámek, J., Herrlich, H., Strecker, G.E. Abstract and Concrete Categories: The Joy of Cats, Dover, 2009. 4

[5] Barr, M., Wells, C. Toposes, Triples and Theories, Springer, 1984. 3

[6] Bénabou, J. Introduction to Bicategories, University of Chicago, 1967. 14

[7] Borceux, F. Handbook of Categorical Algebra, vols. I, II e III, Cambridge University Press, 2008. 7

[8] Cannas da Silva, A., Weinstein, A. Geometric Models for Noncommutative Algebras, University of California, Berkeley, 1998. 57

[9] Crainic, M., Salazar, M.A., Struchiner, I.: Multiplicative forms and Spencer operators, arXiv:1210.2277 [math.DG] (a ser publicado por Math. Z.). 30, 34

[10] Fatibene, L., Francaviglia, M. Natural and Gauge Natural Formalism for Classical Field Theories: A Geometric Perspective including Spinors and Gauge Theories, Springer, 2003. 45, 46, $47,48,51$

[11] Forger, M., Kellendonk, J.: Classical BRST cohomology and invariant functions on constraint manifolds I, Commun. Math. Phys. 143 (1992) 235-251. 57

[12] Forger, M., Römer, H.: Currents and the energy-momentum tensor in classical field theory: a fresh look at an old problem, Ann. Phys. 309 (2004) 306-389. 51

[13] Forger, M., Soares, B.L.: Local symmetries in gauge theories in a finite-dimensional setting, J. Geom. Phys. 62 (2012) 1925-1938. 2, 40, 52

[14] Freyd, P., Scedrov, A. Categories, Allegories, North-Holland, Amsterdam, 1990. 3

[15] Gancarzewicz, J.: Geometries defined by pseudogroups of transformations, Zeszyty Nauk. Uniw. Jagiellon. Prace Mat. 23 (1982) 43-49. 57

[16] Gotay, M.J., Isenberg, J., Marsden, J.E., Montgomery, R.: Momentum maps and classical fields, part I: covariant field theory, arXiv: physics/9801019. 31, 47, 52

[17] Higgins, P., Mackenzie, K.: Algebraic constructions in the category of Lie algebroids, J. Algebra 129 (1990) 194-230. 32, 33 
[18] Hodges, W. Model Theory, Cambridge University Press, 2008. 3

[19] Johnstone, P.T. Sketches of an Elephant: A Topos Theory Compendium, vols. I e II, Oxford University Press, 2003. 3, 14

[20] Kobayashi, S. Transformation Groups in Differential Geometry, Springer-Verlag, 1972. 28, 57

[21] Kobayashi, S., Nomizu, K. Foundations of Differential Geometry, vol. I, Wiley-Interscience, 1996. 12,22

[22] Kock, A., Synthetic Differential Geometry, Cambridge University Press, Cambridge, 2006. 1

[23] Kock, A., Synthetic Geometry of Manifolds, Cambridge University Press, Cambridge, 2009. 1

[24] Kolář, I.: A geometrical version of the higher order Hamilton formalism in fibred manifolds, J. Geom. Phys. 1 (1984) 127-137. 48

[25] Kolár̆, I., Michor, P., Slovák, J. Natural Operations in Differential Geometry, Springer-Verlag, Berlin, 1993. 22, 24, 27, 34, 35, 36, 37, 38, 41, 51, 52

[26] Kosmann-Schwarzbach, Y., Mackenzie, K.: Differential operators and actions of Lie algebroids, Quantization, Poisson brackets and beyond, Contemp. Math. 315 (2002) 213-233. 32, 33, 34

[27] Krasil'schchik, I.S., Lychagin, V.V., Vinogradov, A.M., Geometry of Jet Spaces and Nonlinear Differential Equations, Advanced Studies in Contemporary Mathematics, 1, Gordon and Breach, 1986. 32, 57

[28] Kumpera, A., Spencer, D.C. Lie Equations: Vol. I, General Theory, Princeton University Press, 1972. 24, 28, 33

[29] Mackenzie, K., General Theory of Lie Groupoids and Lie Algebroids, Cambridge University Press, Cambridge, 2005. 21, 22, 23, 24, 32, 43

[30] Mac Lane, S. Categories for the Working Mathematician, Springer-Verlag, New York, 1971. $4,5,14$

[31] Mac Lane, S., Moerdijk, I. Sheaves in Geometry and Logic: A First Introduction to Topos Theory, Springer, 1994. 18

[32] Moerdijk, I., Reyes, G., Models for Smooth Infinitesimal Analysis, Springer-Verlag, New York, 1991. 1

[33] Pêgas, L.H.: Groupoids and cogroupoids: an one object approach, arXiv:1207.3694v2 [math.CT]. 12

[34] Rinehart, G.: Differential forms for general commutative algebras, Trans. Amer. Math. Soc. 108 (1963) 195-222. 31

[35] Roberts, D.M.: Internal Categories, Anafunctors and Localisations, Theory and Applications of Categories 26-29 (2012) 788-829. 18

[36] Salazar, M.A.: Pfaffian Groupoids, Ph.D. thesis, arXiv:1306.1164 [math.DG]. 26, 28, 30, 34, 41,43

[37] Saunders, D.J., The Geometry of Jet Bundles, Cambridge University Press, 1989. 43, 55

[38] Schreiber, U., Waldorf, K.: Parallel transport and functors, J. Homotopy Relat. Struct. 4 (2009) 187-244. arxiv:0705.0452 12 